\title{
UNIVERSITY OF CALIFORNIA \\ Los Angeles
}

Optimum Design of Columns Supported by

Tension Ties
A thesis submitted in partial satisfaction of the requirements for the degree Master of science in Engineering

by

Hagen Richard Mauch

Committee in charge:

Professor Lewis P. Felton, Chairman

Professor William T. Thomson

Professor Earl A. Coddington 
Reproduction Rights Reserved

National Aeronautics and Space Administration

Contract Number NsG-423 
The thesis of Hagen Richard Mauch is approved:

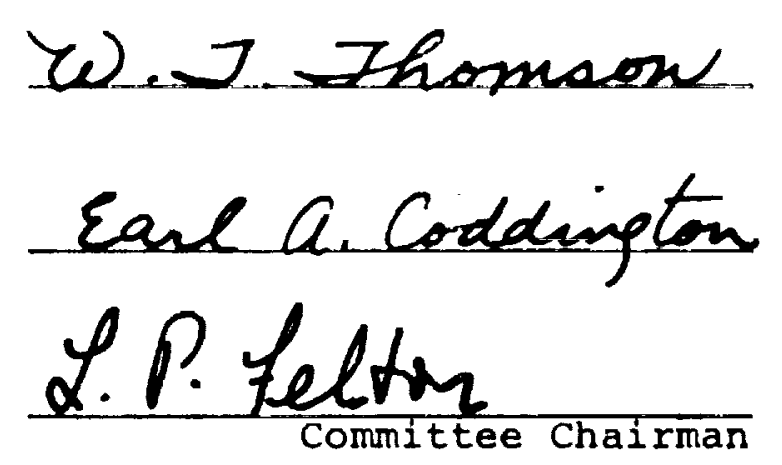

University of California, Los Angeles

1966 
TABLE OF CONTENTS

PAGE

NOMENCLATURE ..................... vii

ABSTRACT OF THE THESIS............. xi

SECTION

1

INTRODUCTION. ................... 1

2 GENERAL THEORY OF THE COLUMN SUPPORTED

BY TENSION TIES................. 5

2.1 Force and deformation relationship

up to the instant of buckling..... 5

2.1 .1 Pretension.............. 5

2.1.2 Relationship between external

load and internal forces... 6

2.2 Lateral reactions produced by tension

ties at buckling load.......... 8

2.3 Lateral reactions when some ties are

relaxed at the instant of buckling.. 10

2.4 Buckling theory............... 13

3 OPTIMUM DESIGN OF THE SIMPLE COLUMN..... 16

4 OPTIMUM DESIGN OF THE COLUMN WITH ONE

STRUT.............................. 18

4.1 Buckling theory for the column with

one strut..................... 18

4.2 Weight assumptions............. 24 
TABLE OF CONTENTS

(continued)

SECTION

PAGE

4

4.2 .1 Weight of the column..... 24

4.2.2 Weight of the wires...... 24

4.2.3 Weight of the strut

assembly............. 25

4.3 Optimization............... 28

4.4 Method of solution............ 29

5 OPTIMUM DESIGN OF THE COLUMN WITH

THREE STRUTS.................. 31

5.1 Buckling theory for the column

with three struts............

5.1.1 Symmetric mode shape

$\left(y_{2}>y_{1}\right) \ldots \ldots \ldots \ldots \ldots \ldots \ldots$

5.1 .2 Symmetric mode shape

$\left(y_{2}<y_{1}\right) \ldots \ldots \ldots \ldots \ldots \ldots . \ldots . \ldots . \ldots$

5.1.3 Antisymmetric mode shape.. 38

5.1.4 Fourth mode shape....... 38

5.2 Buckling theory and optimization. 38

5.2.1 Symmetric mode shape

$\left(y_{2}>y_{1}\right) \ldots \ldots \ldots \ldots \ldots \ldots . \ldots . \ldots . \ldots$

5.2.2 Symmetric mode shape

$\left(y_{2}<y_{1}\right) \ldots \ldots \ldots \ldots \ldots \ldots$. 41 
TABLE OF CONTENTS

(continued)

SECTION

PAGE

5

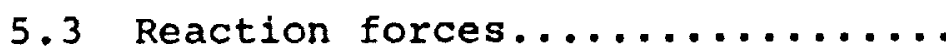

5.3.1 Reaction forces for the

symmetric mode shape.....

5.3.2 Reaction forces for the

asymmetric mode shape....

5.3.3 Reaction forces as func-

tions of buckling force... 44

5.4 Weight assumptions........... 45

5.4.1 Weight of the column...... 45

5.4.2 Weight of the long wires... 45

5.4.3 Weight of the short wires.. 46

5.4.4 Weight of the strut assem-

bly at $x=L / 4$ and $x=3 L / 4 \quad 47$

5.4.5 Weight of the strut assembly at $x=L / 2 \ldots \ldots \ldots \ldots \ldots 48$

5.4.6 Weight of the whole column with three struts........ 49

5.5 optimization............... 50 WITH ONE STRUT................. 56

6.1 Material properties........... 56 6.2 Application for aluminum-alloy.... 56 
TABLE OF CONTENTS

(continued)

SECTION

PAGE

6

6.2 .1 Four tension ties....... 56

6.2.2 Three tension ties....... 59

6.3 Application for steel......... 60

6.3 .1 Four tension ties........ 60

6.3.2 Three tension ties....... 61

7 CALCULATIONS AND RESULTS FOR THE

COLUMN WITH THREE STRUTS.......... 62

7.1 Optimum angle............. 62

7.2 Application for aluminum-alloy... 62

7.2 .1 Four tension ties....... 62

7.2.2 Three tension ties....... 64

7.3 Application for steel......... 65

7.3.1 Four tension ties....... 65

7.3.2 Three tension ties...... 66

7.4 Check of the tension stresses in

the ties................. 67

7.4.1 Tension stresses for a construction with four tension ties.................

7.4.2 Tension stresses for a construction with three tension ties................ 68 
TABLE OF CONTENTS

(continued)

SECTION

PAGE

8

DISCUSSION AND CONCLUSIONS...

70

BIBLIOGRAPHY .

75

APPENDIX I..................
A. Tables
B. Figures

APPENDIX II

Computer Programs and Results 
NOMENCLATURE

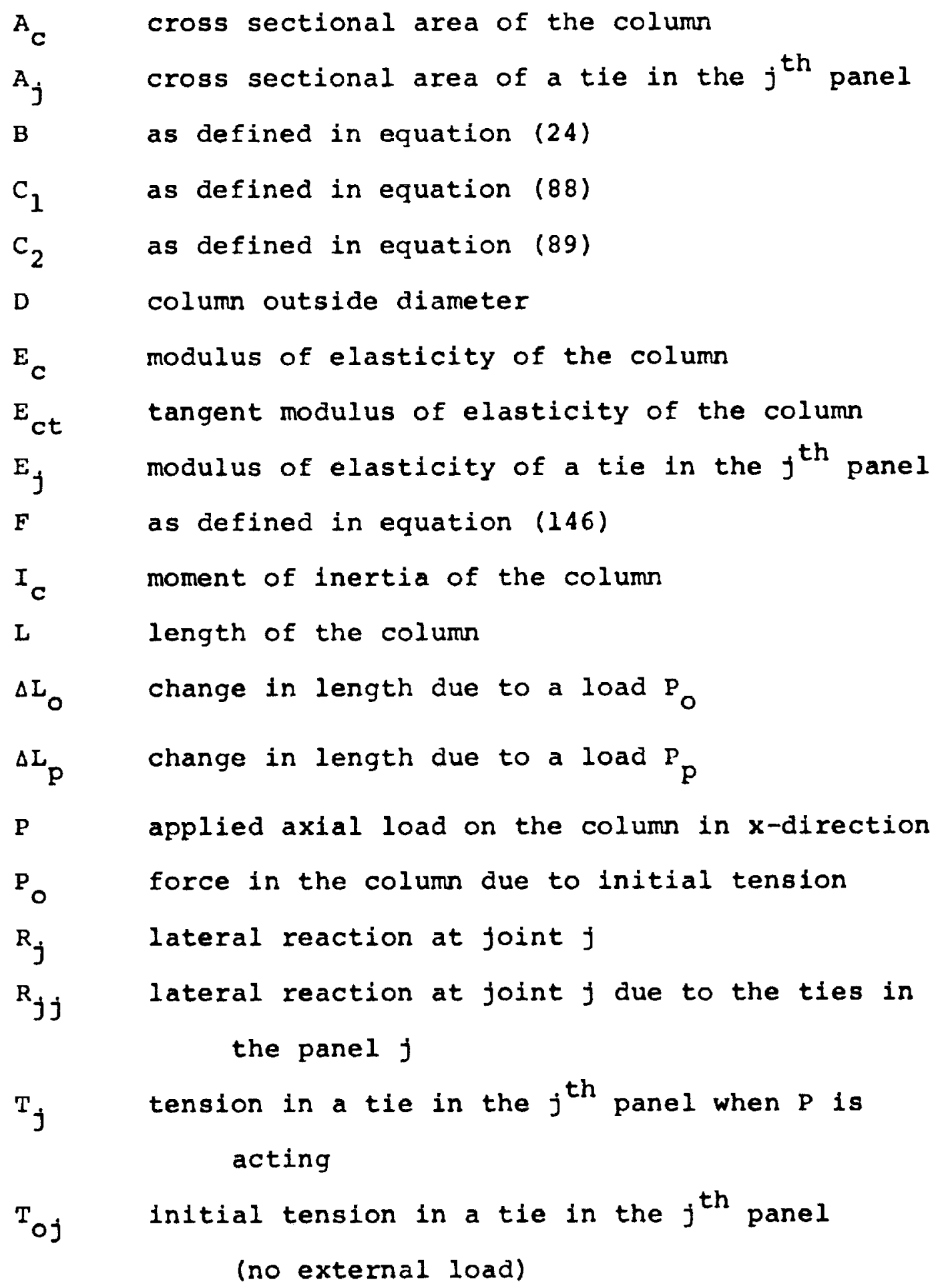


NOMENCLATURE

(continued)

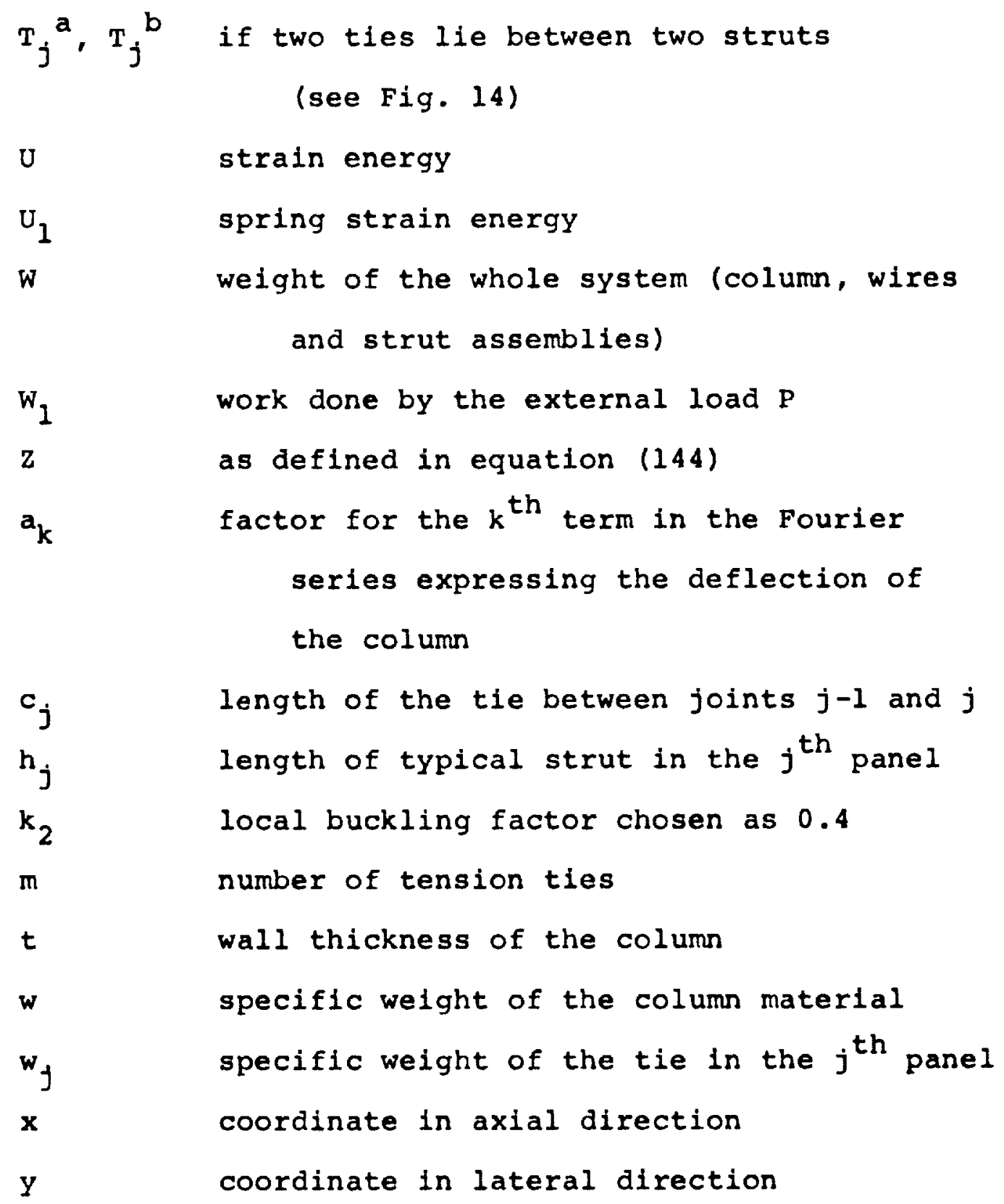




\section{NOMENCLATURE}

\section{(continued)}

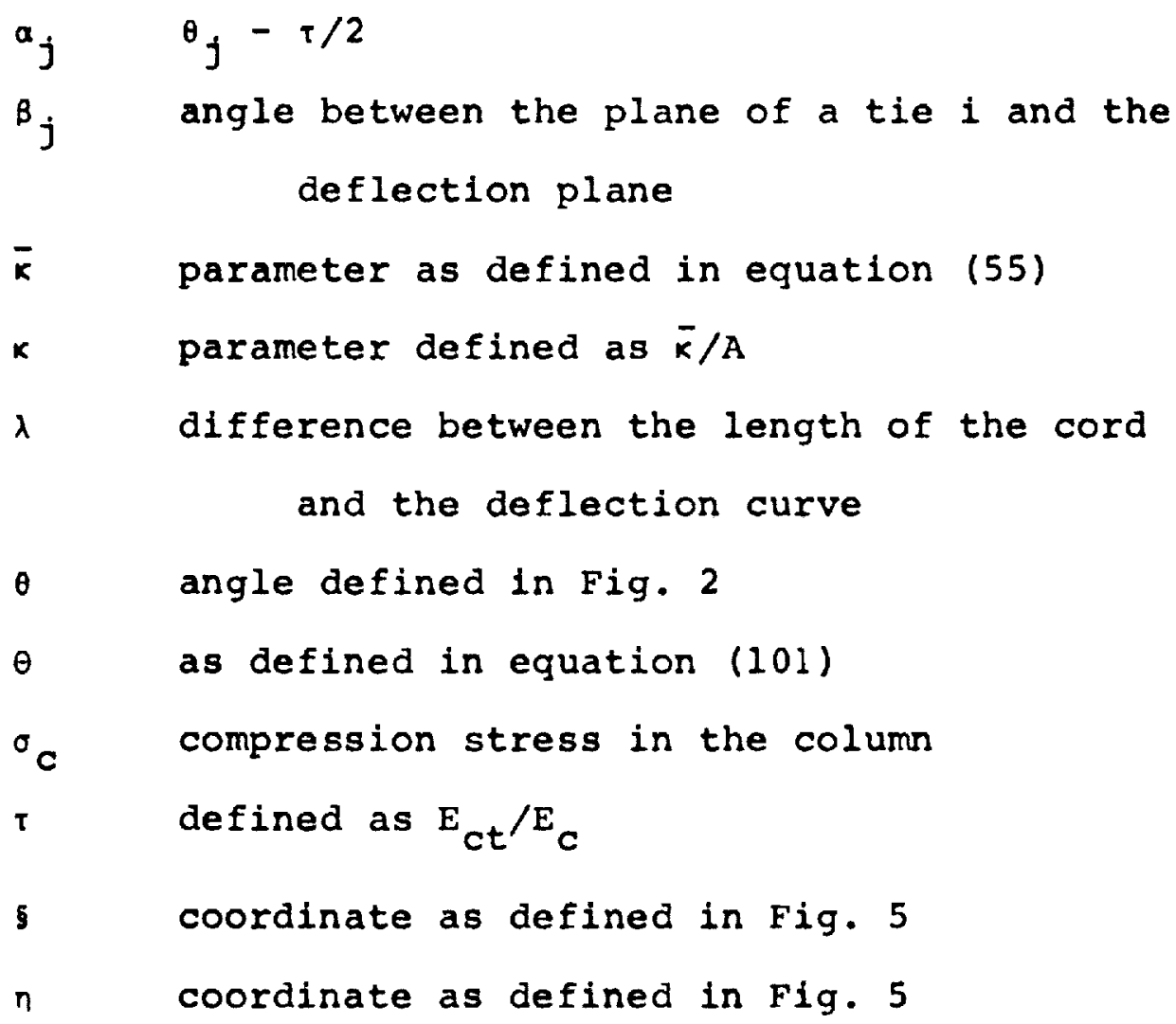




\title{
ABSTRACT OF THE THESIS
}

Optimum Design of Columns Supported by

Tension Ties

by

Hagen Richard Mauch

Master of Science in Engineering

University of California, Los Angeles, 1966

Professor Lewis P. Felton, Chairman

\begin{abstract}
When optimizing simple thin-walled columns on a weight basis, the maximum obtainable stress is found to be that at which local and general buckling failure occur simultaneously. This stress can be expressed as a function of load and distance, allowing the introduction of the
\end{abstract}


structural index $\mathrm{P} / \mathrm{L}^{2} \quad \mathrm{P}=$ buckling load, $\mathrm{L}=$ length of the column), for equal values of which all dimensionally similar columns develop the same stress at failuro. At low values of the structural index, the optimum stress is low, indicating that the simple column is not an efficient structure in such circumstances. It has been found that expansion of the cross-section, for example, by using tension ties which serve to provide intermediate elastic support for the column, allows the column to operate at higher stress levels, thereby increasing efficiency.

This thegis summarizes a method of analysis and presents a procedure for optimizing tension-tie supported thin-walled cylindrical columns. The optimized column for a given structural index is defined by a particular diameter, wall thickness, tie prestress, tie cross-sectional area, tie angle, and strut dimensions. For the cases considered it is found that, in the low range of the structural index, the tie supported column offers a potential weight saving of up to 50 over the simple tubular column. 
SECTION 1

INTRODUCTION

The primary function of a structure is to transmit forces through space, where, from the designer's point of view, the objective often is to do this with the minimum possible weight. For any structure that fails as a result of instability under compressive loading, the maximum obtainable stress depends, in a complex manner, on the properties of the material and the geometric properties of the structure. To apply the principle of dimensional similarity, the structural index $P / L^{2}$ is introduced ( $P$ = buckling load, $L=$ column length). This quantity can be considered as a measure of the loading intensity. All dimensionally similar columns having the same value of structural index will develop the same stress at failure. Therefore, for a particular material and a particular type of cross-section, an easily obtainable relationship between optimum stress and structural index constitutes the information needed for the design of the entire family of minimum weight simple columns.

At low values of the structural index, the optimum stress is far below the elastic limit of most structural materials and an expansion of the cross-section will allow the column to operate at a higher stress level, thereby possibly increasing efficiency, from a weight standpoint. In the age of space technology the long column with small 
compression load becomes more and more interesting and, considering the extremely high cost per pound of orbited load, even the smallest weight savings is appreciated. The thin-walled circular tube, which is the most efficient simple column, is chosen as a basis for the investigation. The weight of the simple column is a minimum when the allowable stress $\sigma$ is a maximum. To find the maximum values of a both primary buckling (Engesser formula) and local buckling are considered and the optimum design is obtained when both failures can occur simultaneously. Using two equal columns and equipping one with tension ties always results in an increase of maximum stress, hence a decrease in weight of the central tube is possible; however, additional weight is added in conjunction with ties and struts. Once the structural index $P / L^{2}$ is specified, the problem is then to define the parameters associated with the tie supported column like column diameter, wall thickness, tie cross-sectional area and tie angle a to obtain minimum weight. The column supported by tension ties considered herein consists of three parts: a thin walled tube with circular cross-section, tension ties, and struts, as shown in Fig. 1. The theory of analyzing such columns has been developed previously [1] and the improved efficiency is proved in tests. [1] Nevertheless in none of the solutions was optimization of the structure with respect to welght attempted, as has been done for the simple 
colums. [2]

For the actual calculations the following assumptions are made:

a. The effect of the deformation of the struts is negligible.

b. The connection between the struts and the column and the connection between the ties and struts are ideal hinges.

c. There is no initial eccentricity or crookedness in the column.

d. There is no lateral deflection before buckling.

e. The pretension in the wires is of such magnitude that at impending buckling the wires are stress free. f. For small lateral deflection the axial deformation is negligible.

g. The angles between the planes of the ties are equal.

h. The struts are distributed symmetrically with respect to the midpoint of the column.

The optimum design can again be found by equating the primary and local buckling stresses. The weight of the column is a function of the above mentioned parameters and a minimization yields optimum values of these parameters. Comparing the optimum weight of the simple column with the optimum weight of the column supported by tension 
ties for identical values of the structural index will

show how much more efficient this supported column can be. 
SECTION 2

GENERAL THEORY OF THE COLUMN SUPPORTED BY TENSION TIES

2.1 Force and deformation relationship up to the instant of buckling

The following theory dealing with the mechanical behavior of the column supported by tension ties is based on Ref. 1 and is repeated here in a slightly modified form for completeness. The geometry of the supported column is sketched in Fig. 1 and Fig. 2 .

\subsubsection{Pretension}

Let the pretension in a tie in the $j^{\text {th }}$ panel be denoted by $\mathrm{T}_{o j}$. If there is no external load applied in the $x$-direction, then the force in the column $\left(P_{0}\right)$ induced by pretension is

$$
P_{0}=m T_{01} \sin \theta_{1}
$$

where $m$ is the number of tension ties. Due to this load, the column has shortened a distance $\Delta L_{0}$.

$$
\Delta L_{0}=\frac{m T_{O L} \sin \theta_{1}}{A_{C} E_{C}} L
$$

From the equilibrium of forces in $x$-direction at any joint, and by neglecting the effect of small angle changes $\Delta \theta_{j}$

$$
T_{0 j}=T_{o l} \frac{\sin \theta_{1}}{\sin \theta_{j}}
$$


The elongation of the tie in the $j^{\text {th }}$ panel due to $T_{0 j}$ is therefore

$$
\Delta c_{o j}=T_{\text {of }} \frac{c_{j}}{A_{j} E_{j}}=T_{\text {ol }} \frac{\sin \theta_{1}}{\sin \theta_{j}} \frac{c_{j}}{A_{j} E_{j}}
$$

By neglecting again small angle changes and with the assumption that there is no lateral deflection before buckling starts, then the component of tie deflection in the $\mathrm{x}$-direction is

$$
\left(\Delta c_{o j}\right)_{x}=\Delta c_{o j} \sin \theta_{j}=T_{o l} \sin \theta_{1} \frac{c_{j}}{A_{j} E_{j}}
$$

and the total displacement in the $\mathrm{x}$-direction is

$$
\left(\Delta c_{0}\right)_{x}=\sum_{j=1}^{n} T_{o l} \sin \theta_{1} \frac{c_{j}}{A_{j} E_{j}}
$$

2.1.2 Relationship between external load and internal forces

Let an external load $P$ be applied to the strut with tension ties that are tightened to a certain value of initial tension. Then the force acting in the column will be increased by the amount $P_{p}(F i g .2)$ and the increase in axial deformation will be

$$
\Delta L_{p}=P_{p} \frac{L}{A_{c} E_{c}}
$$

The force acting in a tie in the $j^{\text {th }}$ panel is decreasing by the amount $\mathrm{T}_{\mathrm{pj}}$. Similar to equation (3) this force is 


$$
T_{p j}=T_{p 1} \frac{\sin \theta_{1}}{\sin \theta_{j}}
$$

The decrease in stretch in the axial direction due to $\mathrm{T}_{\mathrm{pj}}$ is similar to that given by equation $(6)$.

$$
\left(\Delta c_{p}\right)_{x}=\sum_{j=1}^{n} T_{p l} \sin \theta_{1} \frac{c_{j}}{A_{j} E_{j}}
$$

Since $\Delta L_{p}=\left(\Delta c_{p}\right)_{x}$, equations (7) and (9) give

$$
P_{p}=\frac{{ }^{A} c^{E} c}{L} T_{p l} \sin \theta_{1} \sum_{j=1}^{n} \frac{c_{j}}{A_{j} E_{j}}
$$

By taking the summation of the forces in a section at the end of the strut (Fig. 3 ) and considering the fact that the pretension forces in the column and tension ties are in equilibrium regardless of the applied force $P$, it is found that

$$
P=m T_{p 1} \sin \theta_{1}+P_{p}
$$

Substituting $P_{p}$ from equation (10) in equation (11) yields

$$
T_{p l}=\frac{P}{\sin \theta_{1}\left(m+\frac{{ }_{c}{ }^{E} c}{L} \sum_{j=1}^{n} \frac{c_{j}}{A_{j} E_{j}}\right)}
$$

With the assumption that at the onset of buckling the tension ties are stress free, it follows that equations (12) 
and (3) must be equal, or simply

$$
\mathrm{T}_{\text {ol }}=\mathrm{T}_{\mathrm{pl}}
$$

2.2 Lateral reactions produced by tension ties at buckling load

Change in length and slope of the tension ties due to lateral deflection have to be considered next. Let the displacement of joint $j$ in the direction perpendicular to the column and in the plane of column and tie be denoted by $(\Delta y)_{j}$. Then, as shown in Fig. 4 for small displacements, changes in length of the ties may be expressed as

$$
\Delta c_{j}=\left[(\Delta y)_{j-1}-(\Delta y)_{j}\right] \cos \theta_{j}+\left[(\Delta x)_{j-1}-(\Delta x)_{j}\right] \sin \theta_{j}
$$

This is only valid if $\Delta \theta_{j}+0$.

When the lateral deflection starts, the axial deflection $(\Delta x)_{j-1}-(\Delta x)_{j}$ is negligible and may be ignored.

Furthermore, it is assumed that there is no lateral displacement at the end of the column and that the warping of the planes of the ties is negligible. Equation (14) can therefore be simplified to

$$
\Delta c_{j}=\left[(\Delta y)_{j-1}-(\Delta y)_{j}\right] \cos \theta_{j}
$$

and with

$$
\Delta T_{j}=\frac{A_{j} E_{j}}{c_{j}} \Delta c_{j}
$$




$$
\Delta T_{j}=\frac{A_{j} E_{j}}{c_{j}}\left[(\Delta y)_{j-1}-(\Delta y)_{j}\right] \cos \theta_{j}
$$

Now consider the case in which the deflection of the column occurs in the direction 5 (Fig. 4) in the $x-5$ plane. Let $(\Delta s)_{j}$ be the deflection of foint $j$ in the $s$ direction and $(\Delta y)_{j i}$ be the components of $(\Delta 5)_{j}$ in the plane of column and tie $i(i=1,2,3 \ldots \mathrm{m})$. Let $\beta_{i}$ be the angle between the plane of column and tie and the $x-5$ plane. Then referring to Fig. 4

$$
(\Delta y)_{j i}=(\Delta \xi)_{j} \cos \beta_{i} \quad(i=1,2,3,4 \ldots m)
$$

Assuming that the angles between the planes of the ties are equal, then if $\beta_{1}=B$, it follows that

$$
\begin{aligned}
& \beta_{2}=\frac{2 \pi}{m}+\beta \\
& \beta_{3}=\frac{4 \pi}{m}+\beta \\
& \cdots \cdots \\
& \beta_{m}=\frac{m-1}{m} 2 \pi+\beta
\end{aligned}
$$

Let the change in length of the tie $i$ in the panel $j$ be $\left(\Delta c_{j}\right)_{i}$ ! then equation (15) may be written as follows

$$
\left(\Delta c_{j}\right)_{i}=\left(\Delta \xi_{j-1}-\Delta \xi_{j}\right) \cos \theta_{j} \cos \beta_{i}
$$


2.3 Lateral reactions when some ties are relaxed at the instant of buckling

If the ties are stress free at the instant of buckling, some ties are relaxed for an infinitesimal amount of buckling deflection. This gives some constraint to the initial tension which shall be considered later. With this assumption it is clear that ties with an angle $\beta_{i}$ defined by

$$
\pi / 2<B_{1}<3 \pi / 2
$$

will be relaxed and the ties with an angle $B_{i}$ of

$$
-\pi / 2<\beta_{i}<+\pi / 2
$$

are going to be stretched.

For three or four tension ties only one or two ties, but not more than two ties can possibly lay in the region from $-\pi / 2$ to $+\pi / 2$. Therefore in those cases only a maximum of two tension ties can be stretched. Assume now first that tension tie $1=1$ and $1=m$ are stretched. The perpendicular directions to the column axis in each of the planes containing these ties are called $y_{1}$ and $y_{m}$. (Fig. 5, Fig. 6, Fig. 7).

In the direction of $s$, the lateral components of the respective tensile forces produced by the changes in length of the ties are (Fig. 6) 


$$
\begin{aligned}
\left(R_{j j}\right)_{\S 1} & =-\frac{A_{j} E_{j}}{c_{j}}\left(\Delta c_{j}\right)_{1} \cos \beta \cos \theta_{j} \\
& =-\frac{A_{j} E_{j}}{c_{j}}\left[(\Delta \xi)_{j-1}-(\Delta \xi)_{j}\right] \cos ^{2} \theta_{j} \cos ^{2} \beta \\
\left(R_{j j}\right)_{\xi m} & =-\frac{A_{j} E_{j}}{c_{j}}\left[(\Delta \xi)_{j-1}-(\Delta \xi)_{j}\right] \cos ^{2} \theta_{j} \cos ^{2}\left(\frac{m-1}{m} 2 \pi+\beta\right)
\end{aligned}
$$

where the angle $B_{m}$ is replaced by the expression calculated in equation (18).

In order to maintain equilibrium of forces in the $n$ direction, the following condition must be satisfied (Fig. 6)

$$
\left(R_{j j}\right)_{n 1}=\left(R_{j j}\right)_{n m}
$$

with

$$
\begin{aligned}
& \left(R_{j j}\right)_{n 1}=\left(R_{j j}\right)_{\xi 1} \tan \beta \\
& \left(R_{j j}\right)_{n m}=\left(R_{j j}\right)_{\xi m} \tan \left(\frac{m-1}{m} 2 \pi+\beta\right)
\end{aligned}
$$

or

$$
\sin B \cos B=\sin \left(\frac{m-1}{m} 2 \pi+\beta\right) \cos \left(\frac{m-1}{m} 2 \pi+\beta\right)
$$

This equation can only be satisfied for

$$
\beta=\pi / m
$$

With this result the actual direction of deflection is known and this particular 5 direction is denoted as y.(Fig.5) 
The reaction force $R_{j j}$ in this $y$ direction is given by

$$
\begin{aligned}
R_{j j} & =\left(R_{j j}\right)_{y l}+\left(R_{j j^{\prime} y m}\right. \\
& =-\frac{A_{j} E_{j}}{c_{j}}\left[(\Delta y)_{j-1}-(\Delta y)_{j}\right] \cos ^{2} \theta_{j} B
\end{aligned}
$$

where

$$
B=2 \cos ^{2} B / m=2 \cos ^{2}(\pi / m)
$$

If only one tension tie is stretched, there is only one case of equilibrium possible, namely when $\beta=0$. In this case

$$
\begin{aligned}
R_{j j} & =\left(R_{j j}\right)_{y l} \\
& =-\frac{A_{j} E_{j}}{c_{j}}\left[(\Delta y)_{j-1}-(\Delta y)_{j}\right] \cos ^{2} \theta_{j} B
\end{aligned}
$$

where

$$
B=1
$$

The lateral reactions are due to the action of the tles in the $j^{\text {th }}$ panel on strut plane $j$. The reaction on strut plane $j$ due to the ties in the $(j+1)^{\text {th }}$ panel is given by

$$
R_{j(j+1)}=+\frac{A_{j+1} E_{j+1}}{C_{j+1}}\left[(\Delta y)_{j}-(\Delta y)_{j+1}\right] \cos ^{2} \theta_{j+1} B
$$

Therefore the resultant reaction at joint $j$ is obtained by combining equations (24) or (25) with equation (26), as follows: 


$$
R_{j}=R_{j j}+R_{j(j+1)}
$$

or

$$
\begin{aligned}
R_{j}= & \frac{A_{j+1} E_{j+1}}{c_{j+1}}\left[(y)_{j}-(y)_{j+1}\right] \cos ^{2} \theta_{j+1} B \\
& -\frac{A_{j} E_{j}}{c_{j}}\left[(y)_{j-1}-(y)_{j}\right] \cos ^{2} \theta_{j} \text { B }
\end{aligned}
$$

where $\Delta y$ is replaced by $y$, for convenience.

\subsection{Buckling theory}

A strut with tension ties may be considered as a continuous beam on elastic intermediate supports as shown in Fig. 8. In the case of simply supported ends a representation of the deflection curve in the form of a trigonometric series is advantageous.

The deflection curve can be represented as

$$
\begin{gathered}
y=a_{1} \sin \frac{\pi x}{L}+a_{2} \sin \frac{2 \pi x}{L}+a_{3} \sin \frac{3 \pi x}{L}+\ldots \\
a_{k} \sin \frac{k \pi x}{L}
\end{gathered}
$$

Each term satisfies the boundary conditions, since each term together with its second derivative becomes zero at the end of the beam.

For the case of the column supported by tension ties, the coefficients in the above series, and the buckling load, are obtainable from an energy approach. The work done by the longitudinal force $P$ must be equal to the work done 
by the reaction force $R_{j}$ plus the strain energy. The strain energy is defined as

$$
U=\frac{E_{c}{ }^{I} c}{2} \int_{0}^{L}\left(\frac{d^{2} y}{d x^{2}}\right)^{2} d x
$$

Introducing equation (28) and integrating yields

$$
U=\frac{\pi^{4} E_{c} I c}{4 L^{3}} \sum_{k=1}^{k=\infty} k^{4} a_{k}^{2}
$$

Any change in the shape of the deflection curve results in some longitudinal displacement at the hinge $B$. This displacement is equal to the difference between the length of the deflection curve and the length of the chord $A B$ (Fig. 8). In terms of the chosen coefficients this distance is

$$
\lambda=\frac{\pi^{2}}{4 L} \sum_{k=1}^{k \infty \infty} k^{2} a_{k}{ }^{2}
$$

and the work done by the external force $P$ is therefore

$$
W_{1}=\frac{P \pi^{2}}{4 L} \sum_{k=1}^{k=\infty} k^{2} a_{k}^{2}
$$


The displacements at the struts are simply $(y)_{j}$ and the reaction forces $R_{j}$ are linear functions of $(y)_{j}$ [equation (28)]. That means the spring characteristic is linear and the spring strain energy is

$$
U_{1}=\sum_{j=1}^{n-1} 1 / 2 y_{j} R_{j}=1 / 2 \sum_{j=1}^{n-1} R_{j} \sum_{k=1}^{\infty} a_{k} \sin \frac{k \pi x_{j}}{L}
$$

For a conservative system

$$
w_{1}=U+U_{1}
$$

Therefore with equations (31), (33), and (34)

$$
\frac{P \pi^{2}}{4 L} \sum_{k=1}^{\infty} k^{2} a_{k}{ }^{2}=\frac{\pi^{4} E c^{I} c}{4 L^{3}} \sum_{k=1}^{\infty} k^{4} a_{k}{ }^{2}+1 / 2 \sum_{j=1}^{n-1} R_{j} \sum_{k=1}^{\infty} a_{k} \sin \frac{k \pi x_{j}}{L}
$$




\section{SECTION 3}

OPTIMUM DESIGN OF THE SIMPLE COLUMN

The theory of optimum design of the simple column is presented in [2] and the most important results are repeated here for convenience.

The Engesser formula for column buckling is

$$
\sigma_{c r}=\frac{\pi^{2} E_{t}}{(L / \rho)^{2}}
$$

and may be expressed in terms of the structural index $P / L^{2}$, using the proper value of $\rho$ for a thin-walled circular tube

$$
\frac{P}{L^{2}}=\frac{8 \sigma_{c r}^{2}}{\pi E_{t} \frac{D}{t}}
$$

For local buckling

$$
\sigma_{c r}=k_{2} \frac{\sqrt{E E_{t}}}{D / t}
$$

where $\mathrm{k}_{2} \doteq 0.4$

Solving equation (38) for $D / t$ and substituting $\sigma_{c r}$ (1.e., $\sigma_{c c}=\sigma_{c r}$ ) from equation (37) yields an optimal value for $D / t$ :

$$
\left(\frac{D}{t}\right)_{\text {opt }}=2\left[\frac{\mathrm{k}_{2}{ }^{2} \mathrm{E}}{\pi \frac{\mathrm{P}}{\mathrm{L}^{2}}}\right]^{\frac{1}{3}}
$$


Or, in terms of the structural index and the stress $\sigma$, these equations can be combined to yield

$$
\frac{P}{L^{2}}=\frac{8 \sigma^{3}}{\pi k_{2} E^{2} \tau^{3 / 2}}
$$

where $\quad \tau=\frac{E_{t}}{E}$

For any given value of $\sigma$ from a particular stressstrain curve, the value of $\mathrm{P} / \mathrm{L}^{2}$ may be calculated. The weight is obtainable from the relation

$$
\frac{W}{L^{3}}=\frac{P / L^{2} W}{\sigma}
$$

where $w$ is the specific weight. 
4.1 Buckling theory for the column with one strut

From the geometry of the system (Fig. 9) it is seen that

$$
\begin{array}{ll}
{ }_{1}=\pi / 2+\alpha & \cos ^{2} \theta_{1}=\sin ^{2} \alpha \\
\theta_{2}=\pi / 2-\alpha & \cos ^{2} \theta_{2}=\sin ^{2} \alpha
\end{array}
$$

Consider the first three terms of the sine series

$$
y=a_{1} \sin \frac{\pi x}{L}+a_{2} \sin \frac{2 \pi x}{L}+a_{3} \sin \frac{3 \pi x}{L}
$$

and

$$
\begin{aligned}
U & =\frac{T^{4} E_{C} I_{C}}{4 L^{3}}\left[a_{1}{ }^{2}+16 a_{2}{ }^{2}+81 a_{3}{ }^{2}\right] \\
W_{1} & =\frac{P \pi^{2}}{4 L}\left[a_{1}{ }^{2}+4 a_{2}{ }^{2}+9 a_{3}{ }^{2}\right] \\
U_{1} & =\frac{R}{2}\left[a_{1}-a_{3}\right]
\end{aligned}
$$

The reaction force is obtainable from equation (28). With the assumptions that $A_{1}=A_{2}, E_{1}=E_{2}$, and $c_{1}=c_{2}=$ $\frac{L}{2 \cos \alpha}$, then

$$
R=\frac{4 A_{1} E_{1}}{L}\left(a_{1}-a_{3}\right) \sin ^{2} \alpha \cos a B
$$


19

Introducing equation (46) in (45)

$$
U_{1}=\frac{2 A_{1} E_{1}}{L} \sin ^{2} \alpha \cos \alpha B\left[a_{1}{ }^{2}-2 a_{1} a_{3}+a_{3}{ }^{2}\right]
$$

If any coefficient $a_{k}$ in series (39) is given an increase $d a_{k}$, the term $\left(a_{k}+d a_{k}\right) \sin k \pi_{x} / L$ replaces the term $a_{k} \sin k \pi_{x} / L$. This increase $d a_{k}$ in the coefficient $a_{k}$ represents an additional small deflection of the beam given by $d a_{k} \sin k \pi_{x} / L$ superposed upon the original deflection curve.

The change in strain energy of the column, due to the increase $d a_{k}$ is

$$
\frac{\partial U}{\partial a_{k}} d a_{k}=\frac{\pi^{4} E_{c} c_{c}}{2 L^{3}} k^{4} a_{k} d a_{k}
$$

The change in work done by the compression load is

$$
\frac{\partial w_{1}}{\partial a_{k}} d a_{k}=P \frac{\pi^{2} k^{2}}{2 L} a_{k} d a_{k}
$$

and the change in strain energy of the springs is

$$
\frac{\partial U_{1}}{\partial a_{k}} d a_{k}=\frac{2 \sin ^{2} \alpha \cos \alpha A_{1} E_{1} B}{L} \frac{\partial}{\partial a_{k}}\left(a_{1}{ }^{2}-2 a_{1} a_{3}+a_{3}{ }^{2}\right)
$$

For a conservative system

$$
\frac{\partial w_{1}}{\partial a_{k}} d a_{k}=\frac{\partial U}{\partial a_{k}} d a_{k}+\frac{\partial U_{1}}{\partial a_{k}} d a_{k}
$$


For $K=1,2,3$ this results in three equations

$$
\begin{aligned}
\frac{P \pi^{2}}{2 L} a_{1} & =\frac{\pi^{4} E_{c} I_{c}}{2 L^{3}} a_{1}+\frac{4 \sin ^{2} \alpha \cos a A_{1} E_{1} B}{L}\left(a_{1}-a_{3}\right) \\
4 \frac{P \pi^{2}}{2 L} a_{2} & =\frac{\pi^{4} E_{C} I_{c}}{2 L^{3}} 16 a_{2} \\
9 \frac{P \pi^{2}}{2 I} a_{3} & =\frac{\pi^{4} E_{c} I_{c}}{2 L^{3}} 81 a_{3}+\frac{4 \sin ^{2} \alpha \cos \alpha A_{1} E_{1} B}{L}\left(a_{3}-a_{1}\right)
\end{aligned}
$$

Equation (52) states that the second buckling mode of the simple column is a solution of this column supported by tension ties. It can be seen that equations (51) and (53) can only be satisfied if the following determinant is zero.

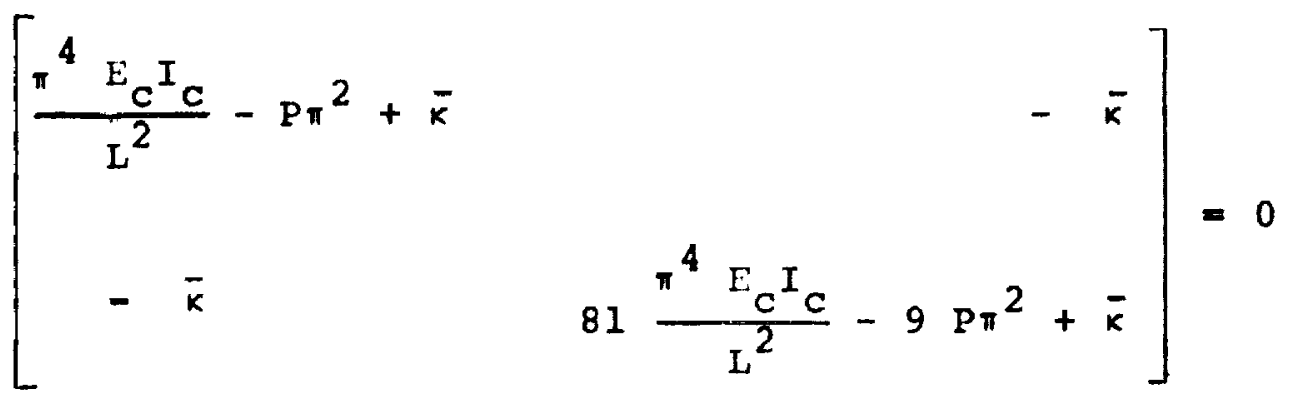

where

$$
\bar{k}=8 A_{1} E_{1} B \sin ^{2} a \cos a
$$


To extend the theory in the plastic region, the modulus of elasticity of the column is replaced by the tangent modulus. Considering, furthermore, local buckling, it can be shown that the moment of inertia $I_{c}$ must be replaced by the expression [2]

$$
I_{C}=\delta^{2} A=k_{1} \frac{P}{\sigma} A=\frac{1}{8 \pi} k_{2} \frac{\sqrt{E E_{t}}}{2} P A
$$

where, as for the simple column, $k_{2} \doteq 0.4$.

Introducing equation (56) in the above determinant, and replacing $E_{c}$ with the tangent modulus $E_{t}$ yields $\left[\begin{array}{cc}\frac{\pi^{3} k_{2}}{8 \sigma^{2}} \tau^{3 / 2} E^{2}\left(\frac{P}{L^{2}}\right)-\sigma \pi^{2}+k & -k \\ -k & 81 \frac{\pi^{3} k_{2}}{8 \sigma^{2}} \tau^{3 / 2} E^{2}\left(\frac{P}{L}\right)-9 \sigma \pi^{2}+\kappa\end{array}\right]=0$

where $\tau=E_{t} / E$ and $k=\frac{\bar{k}}{A}=8 \frac{A_{1} E_{1}}{A} B \sin ^{2} \alpha \cos \alpha$ Expanding equation (57) yields

$$
\begin{aligned}
\left(\frac{P}{L^{2}}\right) & =\frac{8 \sigma^{2}}{81 \pi^{3} k_{2} \tau^{3 / 2} \mathrm{E}^{2}}\left(45 \sigma \pi^{2}-41 k\right) \\
& \pm \sqrt{16 \cdot 81 \pi^{4} \sigma^{2}-90 \cdot 32 \kappa \sigma \pi^{2}+41^{2} \kappa^{2}}
\end{aligned}
$$


Th1s equation should reduce to the solution for the simple column in the limit. For the simple column the cross section of the tension ties is zero, so that $x$ takes the value zero. Substituting $k=0$ in equation (58) yields

$$
\frac{\mathrm{P}}{L^{2}}=\frac{8 \sigma^{3} \pi}{9 \mathrm{k}_{2} \tau^{3 / 2} E^{2}}(5 \pm 4)
$$

Consldering the positive sign in the bracket, the buckling formula for the first mode of the simple column (equation (40)) is obtained. The negative sign yields the buckling load for the third mode shape which is not critical. The tension ties increase the buckling force in all modes, so that the positive sign in equation (58) has to be chosen to obtain the critical load.

For cylindrical tubes the factor $k_{2}$ is chosen as 0.4. K has the same dimensions as the structural index and can be considered as something like a structural index of the elastic support of the column and serves as a parameter in further calculations.

Equations (51) and (53) indicate that the buckling shape resulting from the influence of the tension ties is a combination of the natural first and third buckling mode shape. This buckling mode shall be called "constrained first buckling mode."

Equation (58) can be written in simplied form as 
$\frac{P}{L^{2}}=0.3265 \frac{\sigma^{2}}{\tau^{3 / 2} E^{2}}\left[(10.8325 \sigma-k)+\sqrt{75.0981 \sigma^{2}-16.9097 k \sigma+k^{2}}\right]$

To observe the influence of the tension ties, the "constrained first buckling mode" is calculated for an actual case for some typical values of $x$ assuming a column constructed of 2024-T4 aluminum alloy with material properties as shown in Fig. 10. The result is sketched in Fig. 11 and indicates that for various values of $P / L^{2}$ different values of $k$ result in curves which intersect the curve plotted for the second buckling mode shape. Since at all of these points two modes of failure occur simultaneously, the optimum design is reached when $k$ as a function of $P / L^{2}$ is chosen such that the graphs for the constrained first buckling mode shape and the second buckling mode shape are identical. This relationship can be found, when the structural index for the second mode is introduced in the left hand side of equation (60). The second buckling mode shape is given in equation (52) for the following structural index

$$
\frac{P}{L^{2}}=\frac{2 \sigma^{3}}{\pi K_{2} E^{2} \tau^{3 / 2}}
$$




$$
\begin{gathered}
\text { Combining equations (60) and (61) yields } \\
\kappa=\frac{45}{56} \sigma \pi^{2}=7.93091 \sigma
\end{gathered}
$$

This result is independent of the properties of the chosen material.

\subsection{Weight assumptions}

From Fig. 12 it can be seen that the weight of the column with one strut and $m$ tension ties is the summation of

$$
\begin{aligned}
& w_{1}-\text { the weight of the column } \\
& w_{2}-\text { the weight of the supporting } \\
& \text { wires } \\
& w_{3} \text { - the welght of the strut } \\
& \text { assembly (struts and connecting } \\
& \text { ring) }
\end{aligned}
$$

4.2.1 Weight of the column

As given in equation (41) the weight of the

simple column is

$$
\frac{W_{1}}{L^{3}}=\frac{w \frac{P}{L^{2}}}{\sigma}
$$

4.2.2 Weight of the wires

$$
w_{2}=m w_{1} A_{1} L / \cos \alpha \text { or } \frac{w_{2}}{L^{3}}=\frac{m w_{1} T_{1}}{\sigma_{1} \cos a L^{2}}
$$


$T_{1}$ must be taken as the highest possible tie force. From previous calculations the tie forces are given in the form of equation (16)

$$
\Delta T_{j}=T_{j}=\frac{A_{j} E_{j}}{C_{j}}\left[(\Delta y)_{j-1}-(\Delta y)_{j}\right] \cos \theta_{j}
$$

For the case of only one strut, there is only one possible tensile stress, because $\Delta y_{0}$ and $\Delta y_{2}$ are zero. For $x=L / 2$ the sine series for the deflection gives

$$
y_{1}=\left(a_{1}=a_{3}\right)
$$

Again $\Delta y$ is replaced by $y$ and the angle $\theta$ by $\alpha$. The length of tie 1 is expressed in terms of $L$. Introducing now the value of $y_{1}$ in equation (65) yields

$$
\sigma_{1}=\frac{2 E_{1} \cos a \sin a}{L}\left(a_{1}-a_{3}\right)
$$

This equation gives a constraint for the maximum value of $\sigma$ in the tension ties. For convenience the weight function for the tension ties is written in the form

$$
\frac{W_{2}}{L^{3}}=\frac{m w_{1}}{E_{1} \cos \alpha}\left(\frac{E_{1} A_{1}}{L^{2}}\right)
$$

\subsubsection{Weight of the strut assembly}

The struts are assumed to be simple columns, welded on a ring with a weight $\mathrm{W}_{4}$, which for simplification is chosen to be the same as the weight of one of the struts. The $m$ struts at the midpoint of the column are 
assumed to fail as simply supported columns. Assuming furthermore that buckling occurs elastically

$$
\frac{R}{h^{2}}=\frac{8 \sigma^{3}}{\pi k_{2} E^{2}} \text { and } \sigma^{3}=\frac{\pi k_{2} E^{2} R}{8 h^{2}}
$$

with $\tan \alpha=\frac{2 h}{L}$ it follows that $\frac{1}{h^{2}}=\frac{4}{L^{2} \tan ^{2} \alpha}$

Therefore

$$
\sigma^{3}=\frac{\pi k_{2} E^{2} R}{2 \tan ^{2} \alpha L^{2}}
$$

The weight of the strut assembly is now

$$
w_{3}=(m+1) \text { w A } h=(m+1) w \frac{R}{\sigma} \frac{I \tan a}{2}
$$

and

$$
\frac{W_{3}}{L^{3}}=\frac{m+1}{2} w \frac{\tan \alpha}{\sigma}\left(\frac{R}{L^{2}}\right)
$$

Introducing equation (69) for the optimum stress yields

$$
\frac{W_{3}}{L^{3}}=\frac{m+1}{2} w(\tan \alpha)^{5 / 3}\left(\frac{R}{L^{2}}\right)^{2 / 3}\left[\frac{2}{\pi k_{2} E^{2}}\right]^{1 / 3}
$$

where $R$ is the reaction force given in equation (46). The specific weight of the strut assembly is assumed to be the same as for the column.

Equations (63), (67), and (69) combined give the total weight as 
$\frac{W}{L^{3}}=\frac{W\left(\frac{P}{L^{2}}\right)}{\sigma}+\frac{m w_{1}}{E_{1} \cos \alpha}\left(\frac{A_{1} E_{1}}{L^{2}}\right)+\frac{m+1}{2} w(\tan \alpha)^{5 / 3}\left(\frac{R}{L^{2}}\right)^{/ 3}\left[\frac{2}{\pi k_{2} E^{2}}\right]^{1 / 3}$

Rewriting the equation for the reaction force $R$ yields

$$
\frac{R}{L^{2}}=\frac{4 A_{1} E_{1}}{L^{3}}\left(a_{1}-a_{3}\right) \sin ^{2} \alpha \cos a B
$$

At the onset of buckling, the theory gives only the buckling shape, but does not specify a fixed magnitude of deflection. It is assumed that the struts buckle when $y / L$ reaches a value given by

$$
\frac{y}{I}=\frac{1}{150}
$$

After completing the calculation it must be ascertained whether or not the tension ties are stressed to a value which is below the yield stress. To do so, equation (66) must be used.

With the above assumptions for $y / L$ the reaction

force is

$$
\begin{gathered}
\frac{R}{L^{2}}=\frac{2}{75} \sin ^{2} \alpha \cos \alpha B\left(\frac{A_{1} E_{1}}{L^{2}}\right) \\
\text { Equation (66) takes the form } \\
\sigma_{1}=\frac{E_{1} \cos \alpha \sin \alpha}{75}
\end{gathered}
$$


With the definition of $k$, and equation $(62)$, the following is obtained:

$$
\frac{45}{56} \sigma \pi^{2}=8 \sin ^{2} \alpha \cos \alpha \frac{A_{1} E_{1}}{A} B
$$

or

$$
A_{1} E_{1}=\frac{45 \pi^{2} P}{448 \sin ^{2} \alpha \cos \alpha B}
$$

Introducing equation (75) in (73)

$$
\frac{R}{L^{2}}=\frac{3 \pi^{2}}{5 \cdot 224} \frac{P}{L^{2}}
$$

With equations (75) and (76) the total weight is expressible in terms of $\mathrm{P} / \mathrm{L}^{2}, \sigma$, and $\alpha$ :

$$
\begin{aligned}
\frac{W}{L^{3}} & =\frac{W\left(\frac{P}{L^{2}}\right)}{\dot{\sigma}}+\frac{m w_{1}}{E_{1} \cos \alpha} \frac{45 \pi^{2}}{448 \sin ^{2} \alpha \cos \alpha B}\left(\frac{P}{L^{2}}\right) \\
& +\frac{m+1}{2} w\left(\frac{3 \pi^{2}}{5 \cdot 224} \frac{P}{L^{2}}\right)^{2 / 3}\left[\frac{2}{\pi k_{2} E^{2}}\right]^{1 / 3}(\tan \alpha)
\end{aligned}
$$

\subsection{Optimization}

The minimum weight can be obtained by setting the derivative of equation (77) with respect to a equal to zero.

$$
\frac{\partial}{\partial a} \frac{W}{L^{3}}=0
$$


For simplification, it is assumed that in the following calculations the only cases considered are those for which $w_{1}$ and $w$ are the same. Differentiating equation (77) with respect to a and simplifying the result in such a way that the left hand side of the equation contains terms in a only, yields

$$
\frac{1-2 \sin ^{2} \alpha}{\sin ^{3} \alpha \cos \alpha} \frac{1}{\tan \alpha^{2 / 3}}=\frac{(m+1)}{9 \pi m} E_{1}\left[\frac{56}{75 k_{2} E^{2} P / L}\right]^{1 / 3} B
$$

The term on the left hand side of equation (79) is independent of material properties and the number of tension ties. To find the optimum angle a the function

$$
f(\alpha)=\frac{1-2 \sin ^{2} \alpha}{\sin ^{3} \alpha \cos \alpha} \frac{1}{\tan ^{2 / 3}}
$$

must be calculated for different angles. The result is plotted in Fig. 13. With the help of this figure the optimum angle $\alpha$ can be obtained for all values of $P / L^{2}$.

\subsection{Method of solution}

For any values of the stress $\sigma$ the tangent-modulus ratio $\tau$ can be found from any tangent-modulus curve for typical materials. From equation (61) the structural index for this particular stress value is obtainable. Choosing now the number of tension ties as 3 or 4 , makes it possible to calculate the right hand side of equation (79). The 
optimum value of a can be found now from Fig. 13, and equation (77) can be solved to obtain the optimum weight of the column supported by tension ties in the form $\mathrm{W} / \mathrm{L}^{3}$. 
SECTION 5

OPTIMUM DESIGN OF THE COLUMN WITH THREE STRUTS

In using the theory for more than one strut it can be seen that the second buckling mode is always an independent solution of the "boom problem." Th1s coincides with the solution in $\operatorname{Ref.} 1$, where it was found that, if the intermediate supports are spaced symmetrically with respect to the midpoint, then no matter how many intermediate supporting points are used, the column will always buckle in the second mode.

Nevertheless, it seems to be possible to arrange the tension ties in such a way that the ties influence the load for the second buckling mode also, so that this colums could be designed to have buckling occur in the first, second, third, or fourth mode. A geometry as sketched in Fig. 14 results in a constrained second buckling mode, thereby increasing buckling stresses over the previous case.

In this new problem the following additional assumptions are made:

a. The tension ties $E-1-C-3-A$ and $1-2-3$ have the same cross-section and material properties.

b. The ties $1-C$ and $3-C$ are hinged at point $c$, but cannot move in the $x$-direction. 
Again it is assumed that the tension ties have a pretension of such a magnitude that at the instant of buckling all ties are stress free, but not relaxed.

Instead of two variables, as in the case of one strut, four variables, $a_{1}, a_{2}, A_{1}$, and $A_{2}$, must now be determined. (Fig. 14)

\subsection{Buckling theory for the column with three struts}

The deflection curve must be introduced with four terms and reads

$$
y=a_{1} \sin \frac{\pi x}{L}+a_{2} \sin \frac{2 \pi x}{L}+a_{3} \sin \frac{3 \pi x}{I}+a_{4} \sin \frac{4 \pi x}{I}
$$

$$
\text { For } x=L / 4, L / 2 \text {, and } 3 L / 4 \text { this series yields }
$$

$$
\begin{aligned}
& \left.y\right|_{x=\frac{L}{4}}=y_{1}=\frac{a_{1}}{2} \sqrt{2}+a_{2}+\frac{a_{3}}{2} \sqrt{2} \\
& \left.y\right|_{x=\frac{L}{2}}=y_{2}=a_{1}-a_{3} \\
& \left.y\right|_{x=\frac{3 L}{4}}=y_{3}=\frac{a_{1}}{2} \sqrt{2}-a_{2}+\frac{a_{3}}{2} \sqrt{2}
\end{aligned}
$$

The changes in length of the tension ties, neglecting displacements in the $\mathrm{x}$-direction and assuming $y_{0}=y_{4}=0$ are $(F 1 g \cdot 14)$ 


$$
\begin{array}{rlrl}
\Delta c_{1} & =y_{1} \sin \alpha_{1} & \Delta c_{3}{ }^{a}=\left(y_{3}-y_{2}\right) \sin \alpha_{1} \\
\Delta c_{2}^{a}=\left(y_{1}-y_{2}\right) \sin a_{1} & \Delta c_{3}^{b}=\left(y_{2}-y_{3}\right) \sin \alpha_{2} \\
\Delta c_{2}^{b}=\left(y_{2}-y_{1}\right) \text { sina } & \Delta c_{4}=y_{3} \sin \alpha_{1}
\end{array}
$$

Expressing $y_{1}, y_{2}$, and $y_{3}$ in terms of the series as indicated in equation (82) and using

$$
\Delta T_{j}=\frac{A_{j} E_{j}}{c_{j}} \Delta c_{j}
$$

the following tension stresses in the ties are obtained:

$$
\begin{aligned}
\Delta T_{1} & =\frac{4 A_{1} E_{1}}{L}\left[\frac{a_{1}}{2} \sqrt{2}+a_{2}+\frac{a_{3}}{2} \sqrt{2}\right] \sin a_{1} \cos a_{1} \\
\Delta T_{2}{ }^{a} & =\frac{4 A_{1} E_{1}}{L}\left[a_{1}\left(\frac{\sqrt{2}}{2}-1\right)+a_{2}+a_{3}\left(\frac{\sqrt{2}}{2}+1\right)\right] \sin a_{1} \cos \alpha_{1} \\
\Delta T_{2}{ }^{b} & =-\frac{4 A_{2} E_{2}}{L}\left[a_{1}\left(\frac{\sqrt{2}}{2}-1\right)+a_{2}+a_{3}\left(\frac{\sqrt{2}}{2}+1\right)\right] \sin a_{2} \cos a_{2} \\
\Delta T_{3}{ }^{a} & =\frac{4 A_{1} E_{1}}{L}\left[a_{1}\left(\frac{\sqrt{2}}{2}-1\right)-a_{2}+a_{3}\left(\frac{\sqrt{2}}{2}+1\right)\right] \sin \alpha_{1} \cos \alpha_{1} \\
\Delta T_{3}{ }^{b} & =-\frac{4 A_{2} E_{2}}{L}\left[a_{1}\left(\frac{\sqrt{2}}{2}-1\right)-a_{2}+a_{3}\left(\frac{\sqrt{2}}{2}+1\right)\right] \sin a_{2} \cos \alpha_{2} \\
\Delta T_{4} & =\frac{4 A_{1} E_{1}}{L}\left[\frac{a_{1}}{2} \sqrt{2}-a_{2}+\frac{a_{3}}{2} \sqrt{2}\right] \sin a_{1} \cos \alpha_{1}
\end{aligned}
$$

It can be seen, that the forces $\mathrm{T}^{\mathrm{a}}$ and $\mathrm{T}^{\mathrm{b}}$ have different signs. A negative stress cannot exist in a tension tie, and the above equations must therefore be handled very carefully. 
For the simplified theory three different cases, as shown in Fig. 15, must be considered separately.
a. symmetric buckling shape $\left(y_{2}>y_{1}\right)$
b. antisymmetric buckling shape
c. symmetric buckling shape $\left(y_{2}<y_{1}\right)$

This distinction can be made if the structure has at least one plane of symmetry [5]. For three tension ties this symmetry condition is not fully applicable, because the reaction forces, which are defined as forces in the strut planes and are opposite to the deflection, are dependent on the sign of the deflection. $\quad(B=1$ for $y<0$, $B=1 / 2$ for $Y>0$.$) Nevertheless an approximation is made$ by assuming that symmetric and antisymmetric modes are independent.

\section{1 .1 symmetric mode shape $\left(y_{2}>y_{1}\right)$}

From Fig. 15 it can be seen, that a reaction force $R$ opposing the deflection in all three struts exists and can be written as

$$
\begin{aligned}
& R_{1}=\left[\Delta T_{1} \sin \alpha_{1}-\Delta T_{2}^{b} \sin \alpha_{2}\right] \quad B \\
& R_{2}=\left[\left(\Delta T_{2}{ }^{b}+\Delta T_{3}{ }^{b}\right) \sin \alpha_{2}\right] B \\
& R_{3}=\left[\Delta T_{4} \sin \alpha_{1}-\Delta T_{3}^{b} \sin \alpha_{2}\right] \quad B
\end{aligned}
$$


whereas in the case of a column with one strut, for

$$
\begin{aligned}
& y>0 \quad\left\{\begin{array}{l}
B=1 \text { for four tension ties } \\
B=1 / 2 \text { for three tension ties }
\end{array}\right. \\
& y<0 \quad B=1 \text { for three or four tension ties } \\
& \text { Introducing now equation (83) in (84) yields } \\
& R_{1}=\left[\begin{array}{l}
\frac{4 A_{1} E_{1}}{L}\left\{\frac{a_{1}}{2} \sqrt{2}+a_{2}+\frac{a_{3}}{2} \sqrt{2}\right\} \sin ^{2} \alpha_{1} \cos \alpha_{1} \\
-\frac{4 A_{2} E_{2}}{L}\left\{a_{1}\left(1-\frac{\sqrt{2}}{2}\right)-a_{2}-a_{3}\left(1+\frac{\sqrt{2}}{2}\right)\right\} \sin ^{2} \alpha_{2} \cos a_{2}
\end{array}\right] B \\
& R_{2}=\left[\frac{4 A_{2} E_{2}}{L}\left\{a_{1}(2-\sqrt{2})-a_{3}(2+\sqrt{2})\right\} \sin ^{2} \alpha_{2} \cos a_{2}\right] \\
& R_{3}=\left[\begin{array}{l}
\frac{4 A_{1} E_{1}}{L}\left\{\frac{a_{1}}{2} \sqrt{2}-a_{2}+\frac{a_{3}}{2} \sqrt{2}\right\} \sin ^{2} a_{1} \cos a_{1} \\
-\frac{4 A_{2} E_{2}}{L}\left\{a_{1}\left(1-\frac{\sqrt{2}}{2}\right)+a_{2}-a_{3}\left(1+\frac{\sqrt{2}}{2}\right)\right\} \sin ^{2} a_{2} \cos a_{2}
\end{array}\right]
\end{aligned}
$$


The following notation will be introduced for convenience :

$$
\begin{aligned}
& C_{1}=\frac{4 A_{1} E_{1}}{L} \sin ^{2} \alpha_{1} \cos a_{1} \\
& C_{2}=\frac{4 A_{2} E_{2}}{L} \sin ^{2} a_{2} \cos \alpha_{2}
\end{aligned}
$$

Using these equations the spring strain energy can be computed as

$$
\begin{aligned}
\mathrm{U}_{1}= & 1 / 2\left[\mathrm{R}_{1} \mathrm{y}_{1}+\mathrm{R}_{2} \mathrm{y}_{2}+\mathrm{R}_{3} \mathrm{y}_{3}\right] \\
= & \frac{\mathrm{B}}{2}\left[\mathrm{c}_{1}\left(\mathrm{a}_{1}{ }^{2}+2 \mathrm{a}_{1} \mathrm{a}_{3}+2 \mathrm{a}_{2}{ }^{2}+\mathrm{a}_{3}{ }^{2}\right)\right. \\
& \left.+\mathrm{C}_{2}\left\{\mathrm{a}_{1}{ }^{2}(3-2 \sqrt{2})+2 \mathrm{a}_{2}{ }^{2}-2 \mathrm{a}_{1} \mathrm{a}_{3}+\mathrm{a}_{3}{ }^{2}(3+2 \sqrt{2})\right\}\right]
\end{aligned}
$$

For a virtual displacement of $\mathrm{da}_{k}$ the following equation is again valid

$$
\frac{\partial w_{1}}{\partial a_{k}} d a_{k}=\frac{\partial U}{\partial a_{k}} d a_{k}+\frac{\partial U_{1}}{\partial a_{k}} d a_{k}
$$

As formerly stated there is a clear distinction between symmetric and antisymmetric buckling modes. Therefore in this case only virtual displacements $\mathrm{da}_{\mathrm{k}}$ which are symmetric modes can be applied and the following two equations are obtained. 


$$
\begin{aligned}
\frac{P \pi^{2}}{2 I} a_{1} & =\frac{\pi^{4} E_{t} I_{c}}{2 I^{3} a_{1}+B}\left[C_{1}\left(a_{1}+a_{3}\right)+c_{2}\left\{a_{1}(3-2 \sqrt{2})-a_{3}\right\}\right] \\
g \frac{P \pi^{2}}{2 I} a_{3} & =81 \frac{\pi^{4} E_{t} I_{c}}{2 L^{3}} a_{3}+B\left[c_{1}\left(a_{1}+a_{3}\right)+C_{2}\left\{a_{3}(3+2 \sqrt{2})-a_{1}\right\}\right]
\end{aligned}
$$

\section{1 .2 Symmetric mode shape $\left(y_{2}<y_{1}\right)$}

Agaln, from Fig. 15 the following reaction

forces are obtained

$$
\begin{aligned}
& R_{1}=\left[\Delta T_{1}+\Delta T_{2}^{a}\right] \sin \alpha_{1} B+\Delta T_{2}^{b} \sin \alpha_{2} \\
& R_{2}=-\left[\Delta T_{2}^{b}+\Delta T_{3}^{b}\right] \sin \alpha_{2}-\left[\Delta T_{2}^{a}+\Delta T_{3}^{a}\right] \sin \alpha_{1} B \\
& R_{3}=\left[\Delta T_{4}+\Delta T_{3}^{a}\right] \sin \alpha_{1} B+\Delta T_{3}^{b} \sin _{2}
\end{aligned}
$$

Following the same procedure as before the following two energy equations are obtained

$$
\begin{aligned}
& \frac{P_{\pi}^{2}}{2 L} a_{1}=\frac{\pi^{4} E_{t} I_{c}}{2 L^{3}} a_{1}+4 C_{1} B\left(1-\frac{\sqrt{2}}{2}\right) a_{1}+C_{2}\left[(2 \sqrt{2}-3) a_{1}+a_{3}\right] \\
& 9 \frac{P \pi^{2}}{2 L} a_{3}=\frac{\pi^{4} E_{t} I c}{2 L^{3}} 81 a_{3}+4 C_{1} B\left(1+\frac{\sqrt{2}}{2}\right) a_{3}+C_{2}\left[a_{1}-(2 \sqrt{2}+3) a_{3}\right]
\end{aligned}
$$


5.1.3 Antisymmetric mode shape

$$
\begin{aligned}
& R_{1}=B\left(\Delta T_{1}+\Delta T_{2}^{a}\right) \sin \alpha_{1} \\
& R_{2}=\left(-\Delta T_{2}^{a} B-\Delta T_{3}^{a}\right) \sin \alpha_{1} \\
& R_{3}=\left(\Delta T_{4}+\Delta T_{3}^{a}\right) \sin \alpha_{1}
\end{aligned}
$$

For the antisymmetric mode there is only one virtual displacement possible, namely, a displacement in the second mode.

$$
\begin{aligned}
& 4 \frac{P \pi^{2}}{2 L} a_{2}=\frac{\pi E_{t} I_{c}}{2 L^{3}} 16 a_{2} \\
& +C_{1}\left\{B\left(a_{1}(\sqrt{2}-1)+2 a_{2}+a_{3}(\sqrt{2}+1)\right]-\left[a_{1}(\sqrt{2}-1)-2 a_{2}+a_{3}(\sqrt{2}+1)\right]\right\}
\end{aligned}
$$

\subsubsection{Fourth mode shape}

The fourth mode shape is independent of the tention ties and the following energy equation is valid

$$
\frac{P \pi^{2} 16}{2 L} a_{4}=\frac{\pi^{4} E_{t} I_{c}}{2 L^{3}} 256 a_{4}
$$

\subsection{Buckling theory and optimization}

For the optimum design the tension ties must be arranged in such a way that the first, second, third, and fourth buckling modes occur at the same buckling force $P$, 
which is, in this case, the solution of equation (99). So far, it has not been proved which set of equations, (92) (93) or (95) - (96), is applicable, but the values of $a_{1}$ ' $a_{2}$, and $a_{3}$ can be found, and it can be checked easily whether the assumption $y_{2}>y_{1}$ or $y_{2}<y_{1} 1 s$ valid.

\section{2 .1 Symmetric shape $\left(y_{2}>y_{1}\right)$}

For the assumption that $y_{2}>y_{1}$ equations (92) and (93) together with equation (98) and (99) give a system of four equations which can be solved. Introducing the solution of equation (99) in equation (92), (93), and (98) yields

$$
\begin{aligned}
\frac{15}{2} \theta a_{1}= & B\left\{C_{1}\left(a_{1}+a_{3}\right)+c_{2}\left[a_{1}(3-2 \sqrt{2})-a_{3}\right]\right\} \\
\frac{48}{2} \theta a_{2}= & C_{1}\left\{B\left[a_{1}(\sqrt{2}-1)+2 a_{2}+a_{3}(\sqrt{2}+1)\right]\right. \\
& \left.-\left[a_{1}(\sqrt{2}-1)-2 a_{2}+a_{3}(\sqrt{2}+1)\right]\right\} \\
\frac{63}{2} \theta a_{3}= & B\left\{C_{1}\left(a_{1}+a_{3}\right)+C_{2}\left[a_{3}(3+2 \sqrt{2})-a_{1}\right]\right\}
\end{aligned}
$$

where

$$
\theta=\frac{\pi^{4} E_{t} I_{c}}{L^{3}}
$$


Nontrivial solutions are only obtainable if the following two sub-determinants are zero

$$
\begin{gathered}
2 C_{1}(1+B)-24 \theta=0 \\
\frac{15}{2} \theta-B\left[C_{1}+C_{2}(3-2 \sqrt{2})\right] \\
B\left(C_{2}-C_{1}\right) \\
\frac{63}{2} \theta-B\left[C_{2}-C_{1}\right)
\end{gathered} \mid=0
$$

In solving these two equations the following is obtained

$$
C_{1}=\frac{12 \theta}{1+B} \quad C_{2}=\frac{4.875 \frac{B}{1+B}-2.4609375}{\frac{B}{I+B}-0.511643} \theta
$$

and, for the two different cases under consideration

$$
\begin{array}{lll}
\underline{m=4} & C_{1}=6 \theta & C_{2}=2.01297 \theta \\
\underline{m=3} & C_{1}=8 \theta & C_{2}=0.93762 \theta
\end{array}
$$

Introducing these results in equations (82) and (100) leads to the following

$$
\begin{array}{ll}
\underline{m=4} & a_{1}=3.336734 a_{3} \text { and } y_{2}-y_{1}=-0.72977 a_{3} \\
\underline{m=3} & a_{1}=1.04897 \quad a_{3} \text { and } y_{2}-y_{1}=-1.399857 a_{3}
\end{array}
$$


For a positive deflection, $a_{1}$ must have a positive sign, so that for both cases $y_{2}-y_{1}<0$. That means $y_{2}$ cannot be larger than $y_{1}$ and the solution is physically impossible.

\section{2 .2 Symmetric shape $\left(y_{2}<y_{1}\right)$}

In this case the following two sub-determinants must be zero

$$
\begin{gathered}
2 C_{1}(1+B)-24 \theta=0 \\
\frac{15}{2} \theta-4 C_{1} B\left(1-\frac{\sqrt{2}}{2}\right)-C_{2}(2 \sqrt{2}-3) \\
-C_{2} \\
\frac{63}{2} \theta-4 C_{1} B\left(1+\frac{\sqrt{2}}{2}\right)+C_{2}(2 \sqrt{2}+3)
\end{gathered} \mid=0
$$

For three and four tension ties this results in

$$
\begin{array}{lll}
\underline{m=4} & C_{1}=6 \theta & C_{2}=13.11419 \theta \\
\underline{m=3} & C_{1}=8 \theta & C_{2}=2.805837 \theta
\end{array}
$$

Introducing these results in the energy equations

$$
\begin{array}{ll}
\underline{m=4} & a_{1}=4.82033 \mathrm{a}_{3} \text { and } y_{2}-y_{1}=-0.29521 \mathrm{a}_{3} \\
\underline{m=3} & a_{1}=0.851514 \mathrm{a}_{3} \text { and } y_{2}-y_{1}=-1.4577 \mathrm{a}_{3}
\end{array}
$$

Again, it is obtained that $y_{2}-y_{1}<0$, which agrees with the assumptions made. Equations (110) and (111) are 
therefore valid.

\subsection{Reaction forces}

To calculate the optimum weight, the maximum values of the reaction forces $R_{1}, R_{2}$, and $R_{3}$ are necessary and should be calculated as functions of the buckling load $P$.

\subsubsection{Reaction forces for the symmetric mode}

In this case $a_{2}=0$ and the equations for the reaction forces can be written down with the help of equation (94).

$$
\begin{aligned}
& R_{1}=C_{1}\left[a_{1}(\sqrt{2}-1)+a_{3}(\sqrt{2}+1)\right] B+C_{2}\left[a_{1}\left(1-\frac{\sqrt{2}}{2}\right)-a_{3}\left(\frac{\sqrt{2}}{2}+1\right)\right] \\
& R_{2}=C_{1}\left[a_{1}(\sqrt{2}-2)+a_{3}(\sqrt{2}+2)\right] B+C_{2}\left[a_{1}(\sqrt{2}-2)+a_{3}(\sqrt{2}+2)\right. \\
& R_{3}=C_{1}\left[a_{1}(\sqrt{2}-1)+a_{3}(\sqrt{2}+1)\right] B+C_{2}\left[a_{1}\left(1-\frac{\sqrt{2}}{2}\right)-a_{3}\left(\frac{\sqrt{2}}{2}+1\right)\right]
\end{aligned}
$$

For the two different cases of four or three tension ties the values $c_{1}, c_{2}, B_{1}$, and $a_{3} / a_{1}$ have been calculated previously and therefore the reaction forces can be found as linear functions of $\theta$ and $a_{1}$.

$$
\begin{aligned}
\underline{m}=4 \quad B=1 \quad C_{1}=6 \theta_{j} C_{2}=13.11419 \theta_{j} a_{3}=\frac{1}{4.82033} a_{1} \\
R_{1}=R_{3}=4.687027 \theta a_{1} \\
R_{2}=0.8715523 \theta a_{1}
\end{aligned}
$$


43

$$
\begin{gathered}
\frac{m=3}{B}=1 / 2 \quad C_{1}=8 \theta ; C_{2}=2.805837 \theta_{;} a_{3}=1.174379 a_{1} \\
R_{1}=R_{3}=8.194398 \theta a_{1} \\
R_{2}=-4.088532 \theta a_{1}
\end{gathered}
$$

5.3.2 Reaction forces for the antisymmetric mode

From equation (97) the reaction forces

are obtainable as

$$
\begin{aligned}
& R_{1}=c_{1}\left[a_{1}(\sqrt{2}-1)+2 a_{2}+a_{3}(\sqrt{2}+1)\right] \\
& R_{2}=-C_{1} B\left[a_{1}\left(\frac{\sqrt{2}}{2}-1\right)+a_{2}+a_{3}\left(\frac{\sqrt{2}}{2}+1\right)\right]+\left[a_{1}\left(\frac{\sqrt{2}}{2}-1\right)-a_{2}+a_{3}\left(\frac{\sqrt{2}}{2}+1\right)\right] \\
& R_{3}=c_{1}\left[a_{1}(\sqrt{2}-1)-2 a_{2}+a_{3}(\sqrt{2}+1)\right]
\end{aligned}
$$

Independent of the number of tension ties it is obtrained that

$$
a_{3}=-0.171573 a_{1}
$$

Therefore

$$
\begin{aligned}
\underline{m=4} \quad R_{1}=-R_{3} & =12 \theta a_{2} \\
R_{2} & =0 \\
\underline{m=3} & R_{1}=-R_{3}=8 \theta a_{2} \\
R_{2} & =4 \theta a_{2}
\end{aligned}
$$


5.3.3 Reaction forces as functions of buckling load $P$

As in equation (72) an assumption for the maximum deflection must be made. Introducing the same value as for the case of a column with one strut results in stresses in the tension ties of nearly three times the yield stress of the chosen material. After some trials it is found that the tension stresses reach the yield stresses of the tie material for values of $\mathrm{P} / \mathrm{L}^{2}$, which are calculated for the highest stresses, if $a_{1}=L / 400$ for $m=4$, and $a_{1}=L / 800$ for $m=3$.

Assuming, furthermore, that the reaction force $R_{1}$ is the same for the symmetric and antisymmetric mode gives a constraint for the magnitude of $a_{2}$.

From equation (99)

$$
\theta=\frac{P_{\pi}^{2}}{16 I}
$$

With these assumptions

$$
\begin{aligned}
& \underline{m=4} \quad a_{1}=2.510^{-3} \mathrm{~L} \quad a_{2}=0.97646310^{-3} \mathrm{~L} \\
& \mathrm{R}_{1}=7.227984710^{-3} \mathrm{P} \quad \mathrm{R}_{2}=1.344043210^{-3} \mathrm{P} \\
& \underline{\mathrm{m}=3} \quad \mathrm{a}_{1}=1.2510^{-3} \mathrm{~L} \quad \mathrm{a}_{2}=1.27974910^{-3} \mathrm{~L} \\
& \mathrm{R}_{1}=6.31839610^{-3} \mathrm{P} \quad \mathrm{R}_{2}=3.152515110^{-3} \mathrm{P}
\end{aligned}
$$




\subsection{Weight assumptions}

The weight of the assembled column consists of five parts. A sketch of the parts is shown in Fig. 16 .

5.4.1 Weight of the column

$$
\frac{w_{1}}{L^{3}}=\frac{w \frac{P}{L^{2}}}{\sigma}
$$

5.4.2 Weight of the long wires

$$
w_{2}=\frac{m w_{1} A_{1} L}{\cos \alpha_{1}}
$$

and with equation (88)

$$
\begin{aligned}
E_{1} A_{1} & =\frac{C_{1} L}{4 \sin ^{2} \alpha_{1} \cos \alpha_{1}} \\
\frac{W_{2}}{L^{3}} & =\frac{m w_{1} C_{1}}{4 E_{1} L \sin ^{2} \alpha_{1} \cos ^{2} \alpha_{1}}
\end{aligned}
$$

Again this results in a constraint on $\sigma$ in the tension ties.

$$
\begin{aligned}
& \sigma_{1}=\frac{4 E_{1}}{I}\left[\frac{a_{1}}{2} \sqrt{2}+a_{2}+\frac{a_{3}}{2} \sqrt{2}\right] \cos \alpha_{1} \sin \alpha_{1} \\
& \sigma_{2}=\frac{4 E_{1}}{L}\left[\frac{a_{1}}{2} \sqrt{2}+a_{2}+\frac{a_{3}}{2} \sqrt{2}-a_{1}+a_{3}\right] \cos \alpha_{1} \sin \alpha_{1}
\end{aligned}
$$


46

$$
\begin{aligned}
& \sigma_{3}=\frac{4 E_{1}}{L}\left[\frac{a_{1}}{2} \sqrt{2}-a_{2}+\frac{a_{3}}{2} \sqrt{2}-a_{1}+a_{3}\right] \cos \alpha_{1} \sin \alpha_{1} \\
& \sigma_{4}=\frac{4 E_{1}}{L}\left[\frac{a_{1}}{2} \sqrt{2}-a_{2}+\frac{a_{3}}{2} \sqrt{2}\right] \cos a_{1} \sin \alpha_{1}
\end{aligned}
$$

5.4 .3 Weight of the short wires

$$
w_{3}=\frac{m w_{2} A_{2} L}{2 \cos a_{2}}
$$

from equation (89)

$$
\begin{aligned}
E_{2} A_{2} & =\frac{C_{2} L}{4 \sin ^{2} a_{2} \cos \alpha_{2}} \\
\frac{W_{3}}{L^{3}} & =\frac{m w_{2} C_{2}}{8 E_{2} L \sin ^{2} \alpha_{2} \cos ^{2} \alpha_{2}}
\end{aligned}
$$

The constraints for o are, in this case

$$
\begin{aligned}
& \sigma_{2}=\frac{4 E_{2}}{L}\left[a_{1}-a_{3}-\frac{a_{1}}{2} \sqrt{2}-a_{2}-\frac{a_{3}}{2} \sqrt{2}\right] \sin \alpha_{2} \cos \alpha_{2} \\
& \sigma_{3}=\frac{4 E_{2}}{L}\left[a_{1}-a_{3}-\frac{a_{1}}{2} \sqrt{2}+a_{2}-\frac{a_{3}}{2} \sqrt{2}\right] \sin \alpha_{2} \cos a_{2}
\end{aligned}
$$


5.4.4 Weight of the strut assembly at $x=L / 4$ and $x=3 \mathrm{~L} / 4$

As in the case of the column with one strut assembly, the struts are assumed to be simple columns with circular cross section and shall buckle in the elastic region of the material used.

The optimum buckling load for these simple columns is given by

$$
\frac{R_{1}}{h_{1}^{2}}=\frac{8 \sigma^{3}}{\pi k_{2} E^{2}} \quad \text { and } \quad \sigma=\left[\frac{\pi k_{2} E^{2}}{8} \frac{R_{1}}{h_{1}^{2}}\right]^{1 / 3}
$$

Introducing

$$
h_{1}=\frac{L}{4} \tan \alpha_{1}
$$

yields

$$
\sigma=\left[\frac{2 \pi k_{2} E^{2}}{\tan ^{2} \alpha_{1}}\left(\frac{R_{1}}{L^{2}}\right)\right]^{1 / 3}
$$

The weight of the strut assemblies is now

$$
\begin{array}{rlrl}
\text { at } x & =\mathrm{L} / 4 & \mathrm{w}_{4} \mathrm{I} & =(\mathrm{m}+1) \mathrm{w} \frac{\mathrm{R}_{1}}{\sigma} \frac{\mathrm{It \operatorname {tan } \alpha _ { 1 }}}{4} \\
\text { at } \mathrm{x}=3 \mathrm{~L} / 4 & \mathrm{w}_{4} \mathrm{II} & =(\mathrm{m}+1) \mathrm{w} \frac{\mathrm{R}_{3}}{\sigma} \frac{\mathrm{Itan} \alpha_{1}}{4}
\end{array}
$$


With equation (131) and the consideration that $R_{1}=R_{3}$

$$
\frac{W_{4}}{L^{3}}=\frac{W_{4}{ }^{I}}{L^{3}}+\frac{W_{4}{ }^{I I}}{L^{3}}=\frac{m+1}{2} w\left(\frac{R_{1}}{L^{2}}\right)^{2 / 3}\left[\frac{1}{2 \pi k_{2} E^{2}}\right]^{1 / 3} \tan a_{1} 5 / 3
$$

\subsubsection{Weight of the strut assembly at $x=L / 2$}

Here

$$
\frac{R_{2}}{h^{2}}=\frac{8 \sigma^{3}}{\pi k_{2} E^{2}} \text { and } \sigma=\left[\frac{\pi k_{2} E^{2}}{8} \frac{R_{2}}{h^{2}}\right]^{1 / 3}
$$

Introducing

$$
h=\frac{L}{4}\left(\tan \alpha_{1}+\tan \alpha_{2}\right)
$$

yields

$$
\sigma=\left[\frac{2 \pi k_{2} E^{2}}{\left(\tan \alpha_{1}+\tan \alpha_{2}\right)^{2}} \frac{R_{2}}{L^{2}}\right]^{1 / 3}
$$

The weight of the strut assembly is therefore

$$
\frac{W_{5}}{L^{3}}=\frac{(m+1)}{4} w\left(\frac{R_{2}}{L^{2}}\right)^{2 / 3}\left(\tan \alpha_{1}+\tan \alpha_{2}\right)^{5 / 3}\left[\frac{1}{2 \pi k_{2} E^{2}}\right]^{1 / 3}
$$




\subsubsection{Weight of the whole column with three struts}

As can be seen from Fig. 16

$$
\begin{aligned}
\frac{W}{L^{3}}= & \frac{W_{1}}{L^{3}}+\frac{W_{2}}{L^{3}}+\frac{W_{3}}{L^{3}}+\frac{W_{4}}{L^{3}}+\frac{W_{5}}{L^{3}} \\
= & \frac{W \frac{P}{L^{2}}}{\sigma}+\frac{m w_{1}}{E_{1}} \frac{C_{1}}{4 L \sin ^{2} a_{1} \cos ^{2} a_{1}}+\frac{m w_{2}}{E_{2}} \frac{C_{2}}{8 L \sin ^{2} \alpha_{2} \cos ^{2} \alpha_{2}} \\
& +\frac{(m+1)}{2} w\left(\frac{R_{1}}{L^{2}}\right)^{2 / 3}\left[\frac{1}{2 \pi k_{2} E^{2}}\right]^{1 / 3}\left(\tan \alpha_{1}\right)^{5 / 3} \\
& +\frac{(m+1)}{4} w\left(\frac{R_{2}}{L^{2}}\right)^{2 / 3}\left[\frac{1}{2 \pi k_{2} E^{2}}\right]^{1 / 3}\left(\tan a_{1}+\tan \alpha_{2}\right)^{5 / 3}
\end{aligned}
$$

Assuming again that the tension ties, the column and the struts, are all made from the same material, then the weight equation can be simplified to

$$
\begin{aligned}
\frac{W}{L^{3}}= & {\left[\frac{\frac{P}{L^{2}}}{\sigma}+\frac{m}{8 E_{1} L}\left\{\frac{2 c_{1}}{\sin ^{2} \alpha_{1} \cos ^{2} \alpha_{1}}+\frac{c_{2}}{\sin ^{2} \alpha_{2} \cos ^{2} \alpha_{2}}\right]\right.} \\
& \left.+\frac{m+1}{4}\left\{\frac{1}{2 k_{2} E^{2}}\right\}^{1 / 3}\left[\left(\frac{R}{L^{2}}\right)^{2 / 3} 2\left(\tan \alpha_{1}\right)^{5 / 3}+\left(\frac{R_{2}}{L^{2}}\right)^{\left(\tan a_{1}\right.}+\tan _{2}\right)^{5}\right]
\end{aligned}
$$




\subsection{Optimization}

Since $C_{1}, C_{2}, R_{1}$, and $R_{2}$ are functions of $P$ only, the weight equation is again a function of $\mathrm{P} / \mathrm{L}^{2}$ with the angles $a_{1}$ and $\alpha_{2}$ as parameters. For the optimum design the partial derivatives of equation (137) with respect to $\alpha_{1}$ and $a_{2}$ must be zero.

$$
\frac{\partial}{\partial \alpha 1}\left(\frac{W}{L^{3}}\right)=\frac{\partial}{\partial \alpha 2}\left(\frac{W}{L^{3}}\right)=0
$$

Differentiating equation (137) the following two equations are obtained.

$\frac{m c_{1}}{E_{1} L} \frac{\left(2 \sin ^{2} \alpha_{1}-1\right)}{\cos \alpha_{1} \sin ^{3} \alpha_{1}}+\frac{5(m+1)}{6}\left[\frac{1}{2 \pi k_{2} E^{2}}\right]^{1 / 3}\left\{2\left(\frac{R_{1}}{L^{2}}\right)^{2 / 3} \tan \alpha_{1}^{2 / 3}\right.$

$\left.+\left(\frac{R_{2}}{I^{3}}\right)^{2 / 3}\left(\tan \alpha_{1}+\tan a_{2}\right)^{2 / 3}\right\}=0 \quad$ (139)
$\frac{m c_{2}}{E_{1} L} \frac{\left(2 \sin ^{2} a_{2}-1\right)}{\cos a_{2} \sin ^{3} a_{2}}+\frac{5(m+1)}{3}\left[\frac{1}{2 \pi k_{2} E^{2}}\right]^{1 / 3}\left(\frac{R_{2}}{L^{3}}\right)^{2 / 3}\left(\tan a_{1}+\tan a_{2}\right)=0$

For further calculations all trigonometric functions are expressed as tangent functions. It can be verified that 


$$
\frac{2 \sin ^{2} \alpha-1}{\sin ^{3} \alpha \cos \alpha}=\frac{\tan ^{4} \alpha-1}{\tan ^{3} \alpha}
$$

Equations (139) and (140) are now

$$
\begin{aligned}
& c_{1}=z \frac{\tan ^{3} \alpha_{1}}{1-\tan ^{4} \alpha_{1}}\left[2\left(\frac{R_{1}}{L^{2}}\right)^{2 / 3} \tan \alpha_{1} 2 / 3+\left(\frac{R_{2}}{L^{2}}\right)^{2 / 3}\left(\tan \alpha_{1}+\tan \alpha_{2}\right)^{2 / 3}\right] \\
& C_{2}=22 \frac{\tan ^{3} \alpha_{2}}{1-\tan ^{4} \alpha_{2}}\left[\left(\frac{R_{2}}{L^{2}}\right)^{2 / 3}\left(\tan \alpha_{1}+\tan \alpha_{2}\right)^{2 / 3}\right]
\end{aligned}
$$

where $z$ is defined by

$$
z=\frac{5(m+1)}{6}\left[\frac{1}{2 \pi k_{2} E^{2}}\right]^{1 / 3} \frac{E_{1} L}{m}
$$

For further calculations the cases $m=4$ and $m=3$ must be considered separately.

$\underline{m}=4$

With $m=4$ and $k_{2}$ again chosen as 0.4 , equation (144) yields

$$
Z=0.7661367 \mathrm{E}^{1 / 3} \mathrm{~L}
$$

From equation (118)

$$
\begin{aligned}
& R_{1}=7.227984710^{-3} \mathrm{P} \\
& R_{2}=1.344043210^{-3} \mathrm{P}
\end{aligned}
$$


and from equation (110)

$$
\begin{aligned}
& c_{1}=6 \theta=3.7011018 \frac{P}{L} \\
& c_{2}=13.11419 \theta=8.0894920 \frac{P}{L}
\end{aligned}
$$

With these values equations (142) and (143) can be simplified, and after some algebraic manipulations the following two equations are obtained.

$$
F \frac{1-\tan ^{4} \alpha_{1}}{\tan ^{3} \alpha_{1}}=1.547667 \tan _{1}{ }^{2 / 3}+0.2520965\left(\tan \alpha_{1}+\tan \alpha_{2}\right)^{2 / 3}
$$

$$
\tan \alpha_{1}=9.02438888 \mathrm{~F}^{3 / 2}\left[\frac{1-\tan ^{4} \alpha_{2}}{\tan ^{3} \alpha_{2}}\right]^{3 / 2}-\tan \alpha_{2}
$$

where

$$
F=10^{2}\left[\frac{\frac{P}{L^{2}}}{E}\right]
$$

$\underline{m}=3$

For $m=3$ equation (144) yields

$$
Z=0.8172125 \quad E^{1 / 3} L
$$

From equation (119)

$$
\begin{aligned}
& R_{1}=6.31839610^{-3} \mathrm{P} \\
& R_{2}=3.152515110^{-3} \mathrm{P}
\end{aligned}
$$


and from equation (111)

$$
\begin{aligned}
& C_{1}=80=4.9351108 \frac{P}{L} \\
& C_{2}=2.805837 \theta=1.7307814 \frac{P}{L}
\end{aligned}
$$

With these values, two equations are obtained which must be solved for $a_{1}$ and $a_{2}$.

$$
\begin{aligned}
F \frac{1-\tan ^{4} \alpha_{1}}{\tan ^{3} \alpha_{1}} & =1.1318870 \tan \alpha_{1}{ }^{2 / 3}+0.3560137\left(\tan \alpha_{1}+\tan \alpha_{2}\right)^{2 / 3} \\
\tan \alpha_{1} & =0.34563669 \mathrm{~F}^{3 / 2}\left[\frac{1-\tan ^{4} \alpha_{2}}{\tan ^{3} \alpha_{2}}\right]^{3 / 2}-\tan \alpha_{2}
\end{aligned}
$$

Equations (145), (146), (147), and (148) must be solved for a relatively large number of values of $F$, and because equations are not independent the actual calculations would be very involved. It is therefore advisable that a computer be used to solve the problem. The solution can be obtained by using a "Newton Iteration Process."

$$
x^{(i+1)}=x^{(i)}-\frac{y^{(i)}}{y^{\prime(i)}}
$$

Introducing now the notation

$$
\begin{aligned}
x & =\tan \alpha_{2} \\
x_{1} & =\tan \alpha_{1}
\end{aligned}
$$


$\underline{m=4}$

(Equation (145) can be written as

$$
\begin{aligned}
y & =-x_{1}+\frac{1}{x_{1}^{3}}-\frac{1.547667}{F} x_{1}^{2 / 3}-1.0928455\left(\frac{1}{x^{3}}-x\right) \quad(150) \\
y^{\prime} & =\frac{\partial y}{\partial x}=\frac{\partial y}{\partial x_{1}} \frac{\partial x_{1}}{\partial x} \\
& =-x_{1}{ }^{\prime}-\frac{3}{x_{1}^{4}}-\frac{2 / 31.547667}{F} x_{1}^{-1 / 3} x_{1}^{\prime}+1.0928455\left(1-\frac{3}{x^{4}}\right)
\end{aligned}
$$

From equation $(146), x_{1}$ and $x_{1}$ ' can be expressed in $x$.

$$
\begin{aligned}
& x_{1}=9.025389 \mathrm{~F}^{3 / 2}\left[\frac{1-\mathrm{x}^{4}}{\mathrm{x}^{3}}\right]^{3 / 2}-\mathrm{x} \\
& \mathrm{x}_{1}^{\prime}=3 / 29.024389 \mathrm{~F}^{3 / 2}\left[\frac{1-\mathrm{x}^{4}}{\mathrm{x}^{3}}\right]^{1 / 2}\left(1+\frac{3}{\mathrm{x}^{4}}\right)-1
\end{aligned}
$$

$\underline{m}=3$

In the same way equation (147) becomes

$$
\begin{gathered}
y=-x_{1}+\frac{1}{x_{1}^{3}}-\frac{1.131887}{F} x_{1}^{2 / 3}-0.17535375\left(\frac{1}{x^{3}}-x\right) \\
y^{\prime}=-x_{1}^{\prime}-\frac{3}{x_{1}^{4}}-\frac{2 / 31.131887}{F} x_{1}^{-1 / 3} x_{1}^{\prime}+0.17535375\left(1-\frac{3}{x^{4}}\right)
\end{gathered}
$$


And with equation (148)

$$
\begin{aligned}
& x_{1}=0.34563669 \mathrm{~F}^{3 / 2}\left[\frac{1-\mathrm{x}^{4}}{\mathrm{x}^{3}}\right]^{3 / 2}-\mathrm{x} \\
& \mathrm{x}_{1}^{\prime}=3 / 20.34563669 \mathrm{~F}^{3 / 2}\left[\frac{1-\mathrm{x}^{4}}{\mathrm{x}^{3}}\right]^{1 / 2}\left(1+\frac{3}{\mathrm{x}^{4}}\right)-1
\end{aligned}
$$

For an initial assumed value of $x$ the values of $x_{1}$ and $x_{1}$ ' are obtainable. The three values $x_{1} x_{1}$, and $x_{1}$ ' allow the calculation of $y$ and $y^{\prime}$.

With equation (149) a new value of $x$ is obtained. This procedures has to be repeated until a specified accuracy of $x$ is reached. For the calculation of the cross sectional area, the expressions

$$
\frac{1}{\sin ^{2} \alpha_{1} \cos \alpha_{1}} \text { and } \frac{1}{\sin ^{2} \alpha_{2} \cos \alpha_{2}}
$$

are necessary. Therefore they have also been calculated by the computer. The computer program listed $1 \mathrm{~s}$ in Appendix II of this paper.

With the optimum angles $a_{1}$ and $a_{2}$ calculated, the weight of the column with three struts can be obtained and plotted against the structural index to give the desired results. 


\section{SECTION 6}

CALCULATIONS AND RESULTS FOR THE COLUMN WITH ONE STRUT

\subsection{Material properties}

For the application of the theory developed on the previous pages a typical aluminum-alloy and steel are chosen.

$$
\begin{array}{ll}
\text { Al-alloy } & 2024-\mathrm{T} 4-\mathrm{Al}-\mathrm{Alloy} \\
& \text { (material properties, see Fig. 10) } \\
\text { Steel } & \text { Stainless steel } 3 / 4 \text { hard } \\
& \text { (material properties, see Fig. 17) }
\end{array}
$$

From Ref. 6 the following material data for the tension ties are found.

$$
\begin{array}{ccc}
\text { Al-alloy } & \sigma_{\text {yield }}=60(\mathrm{ksi}) \\
E=10.5 \times 10^{6}(\mathrm{psi}) & W=0.10\left(1 \mathrm{~b} / \mathrm{in} .{ }^{3}\right) \\
\text { Carbon steel } & \sigma_{\text {yield }}=130(\mathrm{ksi}) \quad W=0.283\left(1 \mathrm{~b} / \mathrm{in} .{ }^{3}\right) \\
E=28.3 \times 10^{6} \text { (psi) } &
\end{array}
$$

\subsection{Application for aluminum-alloy}

6.2.1 Four tension ties.

$$
\text { With } m=4 \text { and } B=1 \text { equation (79) takes the }
$$

form

$$
f(\alpha)=\frac{11.9197}{\left(P / L^{2}\right)^{1 / 3}}
$$


Considering the fact that at the instant of buckling the tension ties are stress free as long as there is no lateral deflection, this allows the definition

$$
P=\sigma A
$$

where $A$ is the cross section of the column.

With this relation equation (75) can be modified and gives a relation between wire and column cross sections.

$$
\frac{A}{A}=\frac{8.455410^{-5}}{\sin ^{2} \alpha \cos \alpha} \quad \sigma \quad(k s i)
$$

The weight equation for this particular case is obtained from equation (77)

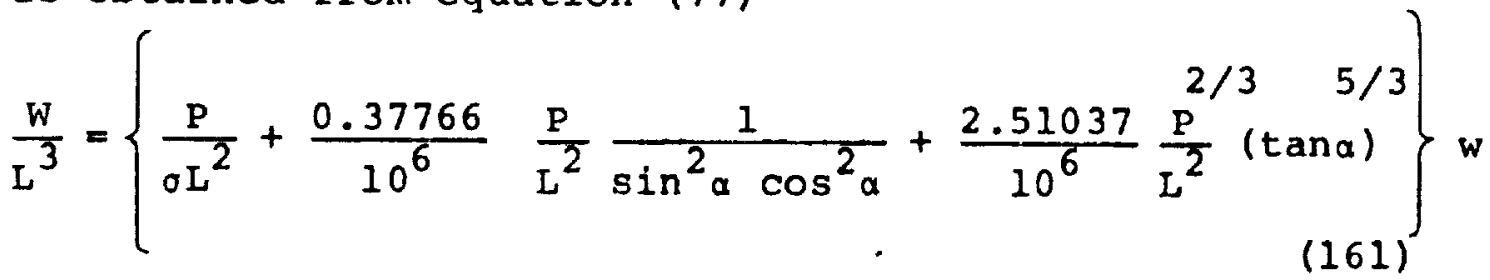

Now all equations necessary for the solution are obtained and the steps which are followed in solving the problem are shown by solving for one value of $\sigma$.

$$
\text { For a stress of } \sigma=20 \text { (ksi) Fig. } 10 \text { gives }
$$

$$
\tau=1.0
$$

Equation (40) gives now the structural index for the simple column

$$
{\frac{P}{L^{2}}}^{(I)}=0.45070(\mathrm{psi})
$$


In the same way equation (61) gives the structural index for the optimum column with one strut.

$$
{\frac{P}{L^{2}}}^{(I I)}=0.11267(p s i)
$$

Now $f(\alpha)$ can be calculated and Fig. 13 gives an optimum value of

$$
a=22.65^{\circ}
$$

The weight of the simple column can be calculated by introducing equation (163) in (41)

$$
\frac{W}{L^{3}}=2.2535\left(1 b / 1 n \cdot{ }^{3}\right)
$$

With equations (164) and (165) all information is obtained to calculate the weight of the column with one strut. Equation (161) yields

$$
\frac{\mathrm{W}}{\mathrm{L}^{3}}=0.6107\left(1 \mathrm{~b} / \mathrm{in} .^{3}\right)
$$

Equation (160) gives

$$
\frac{A_{1}}{A}=1.235510^{-2}
$$

The results for different values of $\sigma$ are noted in Table 1 in Appendix $I$.

The constraint for the tension ties is

$$
\sigma_{1}=\frac{2 E_{1} \cos \alpha \sin \alpha}{150}
$$


For the example

$$
0.2605<\cos \alpha \sin \alpha<0.4655
$$

or

$$
\sigma_{\min }=36.47(\mathrm{ksi}) \quad \sigma_{\max }=65.17 \quad(\mathrm{ksi})
$$

$\sigma_{y i e l d}$ for aluminum wires goes up to 70 (ksi), so that the wires are safe.

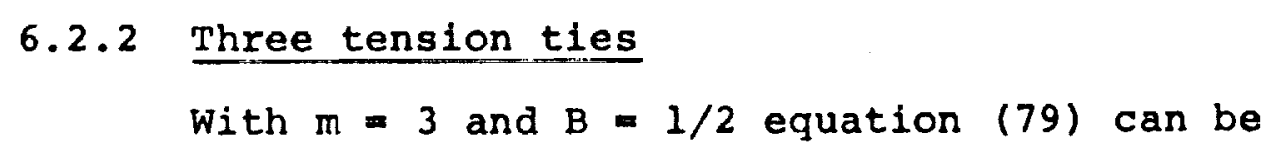

written as

$$
f(\alpha)=\frac{6.3572}{P / L^{2} 1 / 3}
$$

Equation (75) in modified form writes

$$
\frac{{ }^{A} 1}{A}=\frac{16.910810^{-5}}{\sin ^{2} \alpha \cos \alpha} \sigma \quad(k s 1)
$$

The weight equation is

$$
\frac{W}{L^{3}}=W\left\{\frac{P}{\sigma L^{2}}+\frac{0.56649}{10^{6}} \frac{P}{L^{2}} \frac{1}{\sin ^{2} \alpha \cos ^{2} \alpha}+\frac{2.0083}{10^{6}}(\tan \alpha) \frac{5 / 3}{L^{2}}{\frac{p^{2 / 3}}{(173)}}^{2}\right.
$$

The calculations are similar to the calculation in section 6.2.1. The results are noted in Table 2 in the Appendix.

$$
\begin{aligned}
& \text { The stresses in the tension ties are } \\
& \sigma_{\min }=42.77(\mathrm{ksi}) \quad \sigma_{\max }=67.55(\mathrm{ksi})
\end{aligned}
$$


The tension ties are therefore safe.

The various results for a column with one strut, made from aluminum are shown in Fig. (18), (19), and (26).

6.3 Application for steel

$$
\begin{aligned}
6.3 .1 \text { Four tension ties } & \\
\text { With equation } & (79) \\
f(\alpha) & =\frac{16.588}{\left(P / L^{2}\right)^{1 / 3}}
\end{aligned}
$$

From equation (75)

$$
\frac{A_{1}}{A}=\frac{3.101810^{-5}}{\sin ^{2} a \cos \alpha} \quad(k s i)
$$

The weight equation for this case is

$$
\left.\frac{W}{L^{3}}=w\left\{\frac{P}{\sigma L^{2}}+\frac{0.14012}{10^{6}}\left(\frac{P}{L^{2}}\right) \frac{1}{\sin ^{2} a \cos ^{2} \alpha}+\frac{1.2958}{10^{6}}\left(\frac{P}{L^{2}}\right)^{2 / 3}(\tan \alpha)\right\}^{5 / 3}\right\}
$$

The results are noted in Table 3 in Appendix I. The stresses in the tension ties are calculated as

$$
\sigma_{\min }=78.63510^{3}\left(\text { psi) } \quad \sigma_{\max }=176.48510^{3}(\mathrm{psi})\right.
$$

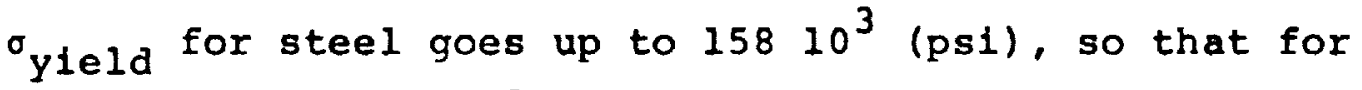
higher values of $\mathrm{P} / \mathrm{L}^{2}$ (over 2.0 ) the deflection should be restricted to a value of $L / 200$. 


\subsubsection{Three tension ties}

The angle $\alpha$, the cross section relation $A_{1} / A$, and the weight $\mathrm{W} / \mathrm{L}^{3}$ can be calculated using the following equations :

$$
f(\alpha)=\frac{8.84693}{\left(P / L^{2}\right)^{1 / 3}}
$$

$$
\frac{A_{1}}{A}=\frac{7.006110^{-5}}{\sin ^{2} \alpha \cos \alpha} \quad(k s i)
$$

$$
\frac{W}{L^{3}}=W\left\{\frac{P}{\sigma L^{2}}+\frac{0.21018}{10^{6}}\left(\frac{P}{L^{2}}\right) \frac{1}{\sin ^{2} a \cos ^{2} a}+\frac{1.03695}{10^{6}}(\tan \alpha)\left(\frac{P}{L^{2}}\right)^{2 / 3}\right\}
$$

The results are noted in Table 4 in Appendix I.

The tension stresses are calculated as

$$
\sigma_{\min }=91.17810^{3}(\mathrm{psi}) \quad \sigma_{\max }=182.73710^{3}(p s i)
$$

Therefore again the deflections of $\mathrm{P} / \mathrm{L}^{2}>2.0$

should be restricted to values of $\mathrm{L} / 200$.

The various results for a column with one strut, made from steel, are shown in Fig. $(20,(21)$, and (31). 
SECTION 7

CALCULATIONS AND RESULTS FOR THE COLUMN WITH THREE STRUTS

\subsection{Optimum angles}

With the computer program given on previous pages, the angles $\alpha_{1}$ and $\alpha_{2}$ are obtained for various values of $F$, which are functions of $\sigma$.

The computed results are printed in Appendix II.

\subsection{Application for aluminum-alloy}

7.2.1 Four tension ties

With the definitions of $c_{1}$ and $c_{2}$ lequations

(88) and (89) l and the results for these variables for the various cases, the cross sections are obtainable. Introducing $E_{1}=E_{2}=10.510^{6}\left(1 \mathrm{~b} / \mathrm{in} .^{2}\right)$ yields

$$
\begin{aligned}
& \frac{A_{1}}{A}=0.088121510^{-3} \frac{\sigma(k s i)}{\sin ^{2} a_{1} \cos \alpha_{1}} \\
& \frac{A_{2}}{A}=0.192606910^{-3} \frac{\sigma(k s i)}{\sin ^{2} a_{2} \cos \alpha_{2}}
\end{aligned}
$$

The weight can be obtained from equation (137) and is written here in such a form that a computer program can be set up easily. 


$$
\begin{aligned}
\frac{W}{L^{3}} 10^{6}=10^{3} 0.105\left\{\frac { P } { L ^ { 2 } } \left[\frac{1}{\sigma}\right.\right. & \left.+\frac{0.35248588}{F_{1}}+\frac{0.38483295}{F_{2}}\right] \\
& \left.+\frac{0.19173222}{10}\left(F_{3}+F_{4}\right)\right\}
\end{aligned}
$$

where

$$
\begin{aligned}
& F_{1}=10^{3} \sin ^{2} \alpha_{1} \cos ^{2} \alpha_{1} \\
& F_{2}=10^{3} \sin ^{2} \alpha_{2} \cos ^{2} \alpha_{2} \\
& F_{3}=2 \tan \alpha_{1}^{5 / 3}\left(\frac{7.2279847}{10^{3}} \frac{P}{L^{2}}\right)^{2 / 3} \\
& F_{4}=\left(\tan \alpha_{1}+\tan \alpha_{2}\right)^{5 / 3}\left(\frac{1.3440432}{10^{3}} \frac{\mathrm{P}}{L^{2}}\right)^{2 / 3}
\end{aligned}
$$

Again the computing of all necessary values for the solution of the problem are indicated for one example. Choosing again a stress of $\sigma=20$ (ksi) the structural index for the optimal column with three struts can be calculated from equation (98).

$$
{\frac{P}{L^{2}}}^{(I V)}=0.028168693
$$

With the help of equations (150), (151), (152), and (153) the computer calculates the optimum angles $a_{1}$ and $\alpha_{2}$ using the above value of the structural index and the modulus of elasticity for aluminum to calculate the value $F$. The following is obtained: 


$$
\begin{aligned}
& a_{1}=25.420^{\circ} \\
& \alpha_{2}=36.012^{\circ}
\end{aligned}
$$

The calculation of $A_{1} / A$ and $A_{2} / A$ can be done now with equations (180) and (181).

$$
\begin{aligned}
& A_{1} / A=1.0590210^{-2} \\
& A_{2} / A=1.3775810^{-2}
\end{aligned}
$$

with this information given, the computer solves equation (182)

$$
\frac{W}{L^{3}} 10^{6}=0.16701
$$

The program for the calculation of the weight is printed in Appendix II for all different cases, discussed in the next three sections.

The results for various values of $\mathrm{P} / \mathrm{L}^{2}$ are noted in Table 5 in Appendix $I$ and in the tables containing the computer results (Appendix II).

\subsubsection{Three tension ties \\ For this case the following two cross section} relations are obtained

$$
A_{1} / A=0.11749510^{-3} \frac{\sigma(k s i)}{\sin ^{2} \alpha_{1} \cos \alpha_{1}}
$$




$$
A_{2} / A=0.04120910^{-3} \frac{\sigma(k s i)}{\sin ^{2} \alpha_{2} \cos \alpha_{2}}
$$

The weight equation is written in a similar form as equation (182).

$$
\begin{aligned}
\frac{W}{L^{3}} 10^{6}=10^{3} 0.105\left\{\frac { P } { L ^ { 2 } } \left[\frac{1}{\sigma}\right.\right. & \left.+\frac{0.35250792}{F_{1}}+\frac{0.618136095}{F_{2}}\right] \\
& \left.+\frac{0.15338577}{10^{4}}\left(F_{3}+F_{4}\right)\right\}
\end{aligned}
$$

where

$$
\begin{aligned}
& F_{1}=10^{3} \sin ^{2} \alpha_{1} \cos ^{2} \alpha_{1} \\
& F_{2}=10^{3} \sin ^{2} \alpha_{2} \cos ^{2} \alpha_{2} \\
& F_{3}=2 \tan \alpha_{1} 5 / 3\left(\frac{6.318396}{10^{3}} \frac{\mathrm{P}}{\mathrm{L}^{2}}\right)^{2 / 3} \\
& F_{4}=\left(\tan \alpha_{1}+\tan \alpha_{2}\right)^{5 / 3}\left(\frac{3.1525151}{10^{3}} \frac{\mathrm{P}}{\mathrm{L}^{2}}\right)^{2 / 3}
\end{aligned}
$$

The results are noted in Table 5 in Appendix I and in the results of the computer calculations (Appendix II).

The various results for a column with three struts made from aluminum-alloy are shown in Figs. (22), (23), (24), (25), and (26).

\subsection{Application for steel}

7.3.1 Four tension ties 
Introducing $\mathrm{E}_{1}=\mathrm{E}_{2}=28.3 \quad 10^{6}\left(\mathrm{lb} / \mathrm{in} .^{2}\right)$

yields here for the cross section relations

$$
\begin{aligned}
& \frac{A_{1}}{A}=0.03269510^{-3} \frac{\sigma(k s i)}{\sin ^{2} \alpha_{1} \cos \alpha_{1}} \\
& \frac{A_{2}}{A}=0.0714619 \quad 10^{-3} \frac{\sigma(k s i)}{\sin ^{2} \alpha_{2} \cos \alpha_{2}}
\end{aligned}
$$

The weight equation reads

$$
\begin{aligned}
\frac{W}{L^{3}} 10^{6}=10^{3} 0.283\left\{\frac{P}{L^{2}\left[\frac{I}{\sigma}\right.}\right. & \left.+\frac{0.13078098}{F_{1}}+\frac{0.14278254}{F_{2}}\right] \\
& \left.+\frac{0.098999204}{10}\left(F_{3}+F_{4}\right)\right\}
\end{aligned}
$$

where $F_{1}, F_{2}, F_{3}$, and $F_{4}$ are defined as in equations (183), $(184),(185)$, and (186).

The results for various values of $\mathrm{P} / \mathrm{L}^{2}$ are noted in Table 6 in Appendix $I$ and in the tables containing the computer results (Appendix II).

\subsubsection{Three tension ties}

The cross section relations are

$$
\begin{aligned}
& \frac{A_{1}}{A}=0.043594 \quad 10^{-3} \frac{\sigma(k s i)}{\sin ^{2} \alpha_{1} \cos \alpha_{1}} \\
& \frac{A_{2}}{A}=0.015289 \quad 10^{-3} \frac{\sigma(k s i)}{\sin ^{2} \alpha_{2} \cos \alpha_{2}}
\end{aligned}
$$


The weight equation is

$$
\begin{aligned}
\frac{W}{L^{3}} 10^{6}=10^{3} \quad 0.283\left\{\frac{\mathrm{P}}{\mathrm{L}^{2}\left[\frac{1}{\sigma}\right.}\right. & \left.+\frac{0.13078916}{\mathrm{~F}_{1}}+\frac{0.02293438}{\mathrm{~F}_{2}}\right] \\
& \left.+\frac{0.079197485}{10}\left(\mathrm{~F}_{3}+\mathrm{F}_{4}\right)\right\}
\end{aligned}
$$

where $F_{1}, F_{2}, F_{3}$, and $F_{4}$ are defined as in equations (196), (197), (198), and (199).

The results for various values of $\mathrm{P} / \mathrm{L}^{2}$ are

noted in Table 7 in Appendix $I$ and in the tables containing the computer results (Appendix II).

The results for a column with three struts are plotted in Figs. (27), (28), (29), (30), and (31).

7.4 Check of the tension stresses in the ties

$$
\text { 7.4.1 Tension stresses for a construction with }
$$

four tension ties

From equation (118) it can be seen that $a_{1}=2.510^{-3} \mathrm{~L}, a_{2}=0.97646310^{-3} \mathrm{~L}, a_{3}=0.207455 \mathrm{a}_{1}$. In looking at equations (123), (124), (125), and (126) it can be seen that only $\sigma_{1}$ is critical. It is obtained

$$
\sigma_{1}=12.4438510^{-3} \mathrm{E}_{1} \cos \alpha_{1} \sin \alpha_{1}
$$

Rquations (128) and (129) again yield only one critical stress $\sigma_{2}$. 


$$
\sigma_{2}=10.462488 \quad 10^{-3} \mathrm{E}_{2} \cos \alpha_{2} \sin \alpha_{2}
$$

The maximum values of the product $\cos \alpha \sin \alpha$ can be calculated from the computer results

Aluminum

$$
\begin{aligned}
& \left(\cos \alpha_{1} \sin \alpha_{1}\right)_{\max }=0.48059 \text { and } \sigma_{1}=62.794 \text { (ksi) } \\
& \left(\cos \alpha_{2} \sin \alpha_{2}\right)_{\max }=0.49867 \text { and } \sigma_{2}=54.782(\mathrm{ksi})
\end{aligned}
$$

Steel

$$
\left(\cos \alpha_{1} \sin \alpha_{1}\right)_{\max }=0.48223 \text { and } \sigma_{1}=169.822(\mathrm{ksi})
$$

$$
\left(\cos \alpha_{2} \sin \alpha_{2}\right)_{\max }=0.49882 \text { and } \sigma_{2}=147.650 \text { (ksi) }
$$

\subsubsection{Tension stresses for a construction with three tension ties}

From equation (119)

$a_{1}=1.2510^{-3} \mathrm{~L}, a_{2}=1.2797510^{-3} \mathrm{~L}, a_{3}=0.58789 \mathrm{a}_{1}$

Again there are two critical stresses $\sigma_{1}$ and $\sigma_{2}$

$$
\begin{aligned}
& \sigma_{1}=10.7305510^{-3} E_{1} \cos \alpha_{1} \sin \alpha_{1} \\
& \sigma_{2}=0.4102810^{-3} E_{2} \cos \alpha_{2} \sin \alpha_{2} \text { (not critical) }
\end{aligned}
$$


With the maximum values of $\cos a_{1} \sin a_{1}$ the maximum tension stresses are obtained as

Aluminum

$$
\left(\cos \alpha_{1} \sin \alpha_{1}\right)_{\max }=0.48566 \text { and } \sigma_{1}=54.720 \text { (ksi) }
$$

Steel

$$
\left(\cos \alpha_{1} \sin \alpha_{1}\right)_{\max }=0.48428 \text { and } \sigma_{1}=147.064(\mathrm{ksi})
$$

The results indicate that the maximum deflections are chosen in such a way that the tension ties are never loaded beyond their yield strength. 


\section{SECTION 8 \\ DISCUSSION AND CONCLUSIONS}

The weight curves Figs. (29) and (31) indicate that for small values of the structural index the column supported by tension ties is up to 508 lighter than the simple column. To demonstrate for which values of the structural index a wire supported column configuration is advisable, a weight savings factor is defined as

weight of the simple column - weight of the wire supported column weight of the simple column

Using the weight curves in Figs. (29) and (31), respectively, this weight saving function can be found graphically and is plotted in Figs. (32) and (33) for the two used materials. There is hardly any difference between the weight of the column with three and four ties, so that the weight figures and the weight saving figures are drawn without the distinction between a column with three and four ties.

During the calculations it was found that the angles $\alpha$ are always smaller than $45^{\circ}$ and in a very reasonable region. The cross sectional area of a tension tie is usually from one to five percent that of the central column. 
Both values (the tie angles and tie cross sections) increase with increasing values of the structural index, where the greatest rate of change occurs for very small values of the structural index. This indicates that the additional weight from the tension ties also increases rapidly for these values of the structural index [Figs. $(18),(20),(22),(23),(27),(28))$.

If typical design parameters ( $P$ and $L$ ) are given, a decision must be made whether a column with three or one strut is chosen. When the weight curves indicate significant weight savings and weight savings are important for a particular problem, it seems reasonable to calculate the column with three struts first. Except for large values of $P$ and very large values of $L$, the wall thickness of the central column usually is too small to be manufactured. In this case the column with one strut must be used. To indicate how the calculations in this paper can be used, a typical practical example is calculated. Assuming that a load of 1000 pounds has to be carried over a distance of 10 feet and that an aluminum construction with one strut and three tension ties is chosen. This allows the calculation of the structural index:

$$
\mathrm{P} / \mathrm{L}^{2}=0.0695 \text { and } \sqrt{\mathrm{P} / \mathrm{L}^{2}}=0.2635
$$


Fig. (18) gives immediately

$$
\begin{aligned}
\alpha & =25.15^{\circ} \\
\frac{A_{1}}{A} & =1.73 \quad 10^{-2}
\end{aligned}
$$

From the expression for the second buckling mode, equation (61), the following maximum stress is obtained

$$
\sigma=\sqrt[3]{\frac{p}{L^{2}} \frac{\pi k_{2} E^{2} T^{3 / 2}}{2}}
$$

for the given values

$$
\frac{\sigma}{\tau}=16.34 \text { (ksi) }
$$

To evaluate $\sigma$ an iteration is usually required. In this case however Fig. (10) yields $\tau=1$, so that

$$
\sigma=16.34(\mathrm{ksi})
$$

From equation (159) it is obtained

$$
A=\frac{P}{\sigma}=\frac{1000}{1.63410^{4}}=0.0612\left(\text { in. }{ }^{2}\right)
$$

Now the cross section of the tension ties is

$$
A_{1}=1.73 \quad 10^{-2} \quad A=0.1059 \quad 10^{-2}\left(\text { in. }{ }^{2}\right)
$$

and for a circular cross section

$$
D_{1}=0.0372 \text { (in.) }
$$


From equation (39) it is obtained

$$
\frac{D}{t}=2\left(\frac{k_{2}^{2} E}{\pi / L^{2}}\right)^{1 / 3}=394.95
$$

With $D / t$ and $A$ given, the wall thickness and column diameter $D$ can be calculated

$$
\begin{aligned}
& t=0.71510^{-2}(\mathrm{in.}) \\
& D=2.825 \text { (in.) }
\end{aligned}
$$

The result for $t$ indicates that a column with three struts would be impossible for this particular case.

The required pretension in the tension ties is obtained from equation (12). Introducing in this equation

$$
\begin{aligned}
\sin \theta_{1} & =\cos \alpha \\
c_{1} & =c_{2}=\frac{L}{2 \cos \alpha} \\
m & =3 \\
E_{1} & =E_{c}
\end{aligned}
$$

the following simple equation is obtained

$$
\mathrm{T}_{01}=\frac{\mathrm{P}}{3 \cos a+\frac{\mathrm{A}_{\mathrm{C}}}{\mathrm{A}_{1}}}=16.53 \text { (1bs) }
$$

The fact that the weight of the optimal wire supported column is nearly independent of the number of tension ties leads to the conclusion that an arrangement 
of four ties does not bring any advantages, so that from the manufacturing point of view the column with three tension ties is preferred. Figures (29) and (31) show that for small values of the structural index $\mathrm{P} / \mathrm{L}^{2}$ the colums supported by tension ties has a significantly smaller weight than the simple column. Nevertheless it should be mentioned that the calculated optimum weight for very small values of $\mathrm{P} / \mathrm{L}^{2}$ cannot be reached in practice because the optimum ratio $D / t$ reaches values which give dimensions of a column which is nearly impossible to fabricate. As is often the case in calculations in optimum design, the results presented in this paper have to be considered as the theoretical limit. 
1. Chu, Kuang-Hna and Berge, Sigmon S. "Analysis and Design of Struts with Tension Ties," Journal of the Structural Division, Proceedings of the American Society of Civil Engineers, Vol. No. 89, February 1963, pp. 127-163.

2. Shanley, F. R. Weight-Strength Analysis of Aircraft Structures, New York, Dover Publications, 1960, Second Edition.

3. Timoshenko, Stephen P. and Gere, James M. Theory of Elastic Stability, New York, McGraw-Hill, 1961, Second Edition.

4. Shanley, F. R. Strength of Materials, New York, McGraw-Hill, 1957 .

5. The American Institute of Mining and Metallurgical Engineers, Institute of Metals Division. Rod and Wire Production Practice, New York, 1949.

6. Hurty, Walter C. and Rubinstein, Moshe F. Dynamics of Structures, Englewood Cliffs, New Jersey, Prentice-HalI, Inc., 1964. 
APPENDIX I

Tables and Figures 
TABLE 1 (a)

Colums with one strut, four tension ties, made from aluminum-alloy.

\begin{tabular}{|c|c|c|c|c|c|}
\hline $\begin{array}{c}\sigma \\
{[k s i]}\end{array}$ & $\begin{array}{l}\mathrm{P} / \mathrm{L}^{2(\mathrm{I})} \\
{[\mathrm{psi}]}\end{array}$ & $\sqrt{\mathrm{P} / \mathrm{L}^{2}}{ }^{(\mathrm{I})}$ & $\begin{array}{l}P / \mathrm{I}^{2(\mathrm{II})} \\
{[\mathrm{psi}]}\end{array}$ & $\sqrt{\mathrm{P} / \mathrm{L}^{2}}$ & $\begin{array}{c}W / L^{3} \\
\text { simple column } \\
{\left[1 \mathrm{~b} / \mathrm{inch}^{3}\right]}\end{array}$ \\
\hline 5 & 0.00704 & 0.08391 & 0.00176 & 0.0419 & 0.1408 \\
\hline 10 & 0.05634 & 0.2374 & 0.01408 & 0.1187 & 0.5634 \\
\hline 15 & 0.19014 & 0.4365 & 0.04753 & 0.2182 & 1.2676 \\
\hline 20 & 0.45070 & 0.6713 & 0.11267 & 0.3356 & 2.2535 \\
\hline 25 & 0.88028 & 0.9382 & 0.22007 & 0.4691 & 3.5211 \\
\hline 30 & 1.6527 & 1.2855 & 0.41317 & 0.6427 & 5.5090 \\
\hline 35 & 3.5038 & 1.8718 & 0.87595 & 0.9359 & 10.0109 \\
\hline 40 & 11.1626 & 3.3410 & 2.7906 & 1.6705 & 27.9065 \\
\hline 45 & 53.2218 & 7.2953 & 13.3054 & 3.6476 & 118.2706 \\
\hline 50 & 185.1575 & 13.606 & 46.2894 & 6.803 & - \\
\hline
\end{tabular}


TABLE 1 (b)

Column with one strut, four tension ties, made from aluminum-alloy.

\begin{tabular}{|c|c|c|c|}
\hline $\begin{array}{c}\sigma \\
{[\mathrm{ksi}]}\end{array}$ & $a^{0}$ & $\begin{array}{c}\mathrm{W} / \mathrm{L}^{3} \\
{\left[\mathrm{Ib} / \mathrm{inch}^{3}\right]}\end{array}$ & $\mathrm{A}_{1} / \mathrm{Ax} 10^{2}$ \\
\hline 5 & 15.70 & 0.0366 & 0.5997 \\
10 & 19.20 & 0.1488 & 0.8278 \\
15 & 21.20 & 0.3395 & 1.0403 \\
20 & 22.65 & 0.6107 & 1.2355 \\
25 & 23.85 & 0.9645 & 1.4136 \\
30 & 24.95 & 1.5225 & 1.5726 \\
35 & 26.38 & 2.8124 & 1.6729 \\
40 & 28.72 & 7.7527 & 1.6705 \\
45 & 31.83 & 32.709 & 1.6098 \\
50 & 34.30 & 102.356 & 1.6115 \\
\hline
\end{tabular}


TABLE 2

Column with one strut, three tension ties, made from aluminum-alloy.

\begin{tabular}{|c|c|c|c|}
\hline$\left[\begin{array}{c}\sigma \\
k s i\end{array}\right]$ & $\alpha^{0}$ & $\begin{array}{c}W / L^{3} \\
{\left[1 b / \text { inch }^{3}\right]}\end{array}$ & $A_{1} / A \times 10^{2}$ \\
\hline 5 & 18.83 & 0.0367 & 0.8573 \\
\hline 10 & 22.30 & 0.1499 & 1.2694 \\
\hline 15 & 24.45 & 0.3429 & 1.6266 \\
\hline 20 & 26.12 & 0.6185 & 1.9438 \\
\hline 25 & 27.45 & 0.9794 & 2.2420 \\
\hline 30 & 28.70 & 1.5500 & 2.5080 \\
\hline 35 & 30.22 & 2.8392 & 2.6842 \\
\hline 40 & 32.50 & 7.9345 & 2.7782 \\
\hline 45 & 35.42 & 33.5885 & 2.7804 \\
\hline 50 & 37.40 & 105.4972 & 2.8851 \\
\hline
\end{tabular}




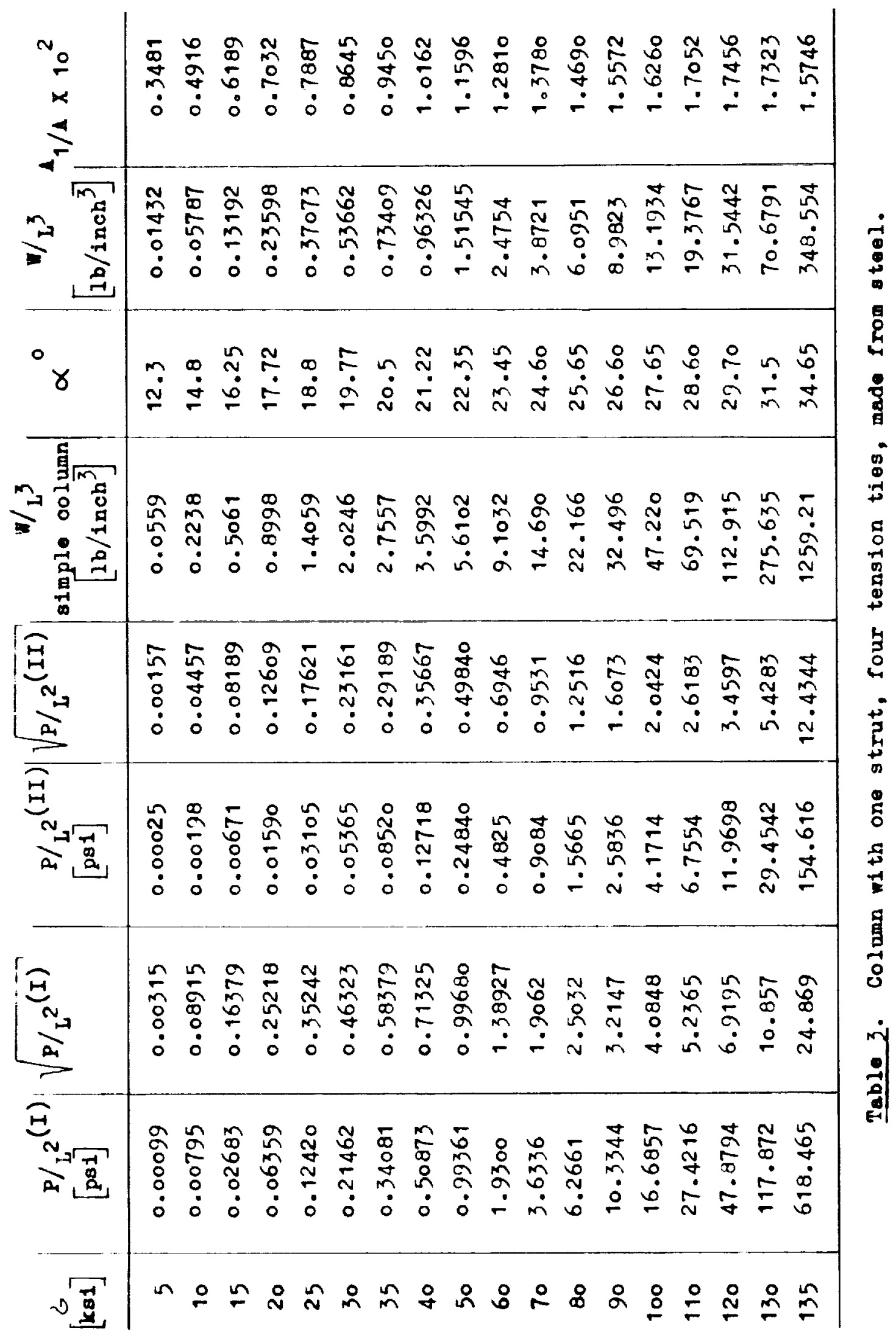


TABLE 4

Column with one strut, three tension ties made from steel.

\begin{tabular}{|c|c|c|c|}
\hline$\stackrel{\sigma}{[k s i]}$ & $a^{0}$ & $\begin{array}{c}W / L^{3} \\
{\left[1 \mathrm{~b} / \text { inch }^{3}\right]}\end{array}$ & $A_{1} / A \times 10^{2}$ \\
\hline 5 & 14.45 & 0.01435 & 0.5809 \\
\hline 10 & 17.35 & 0.05808 & 0.8254 \\
\hline 15 & 19.40 & 0.13243 & 1.0099 \\
\hline 20 & 20.80 & 0.23722 & 1.1886 \\
\hline 25 & 22.0 & 0.37319 & 1.3461 \\
\hline 30 & 22.85 & 0.54095 & 1.5125 \\
\hline 35 & 23.70 & 0.74073 & 1.6575 \\
\hline 40 & 24.45 & 0.97304 & 1.7970 \\
\hline 50 & 25.72 & 1.53041 & 2.0650 \\
\hline 60 & 27.0 & 2.50986 & 2.2890 \\
\hline 70 & 28.27 & 4.0809 & 2.4828 \\
\hline 80 & 29.35 & 6.2036 & 2.6766 \\
\hline 90 & 30.35 & 9.1587 & 2.8620 \\
\hline 100 & 31.35 & 13.3948 & 3.0294 \\
\hline 110 & 32.30 & 19.8377 & 3.1932 \\
\hline 120 & 33.43 & 32.3650 & 3.3186 \\
\hline 130 & 35.15 & 72.7204 & 3.3607 \\
\hline 135 & 37.80 & 359.551 & 3.1865 \\
\hline
\end{tabular}


TABLE 5

Cross-sections for a column with three struts made from aluminum-alloy.

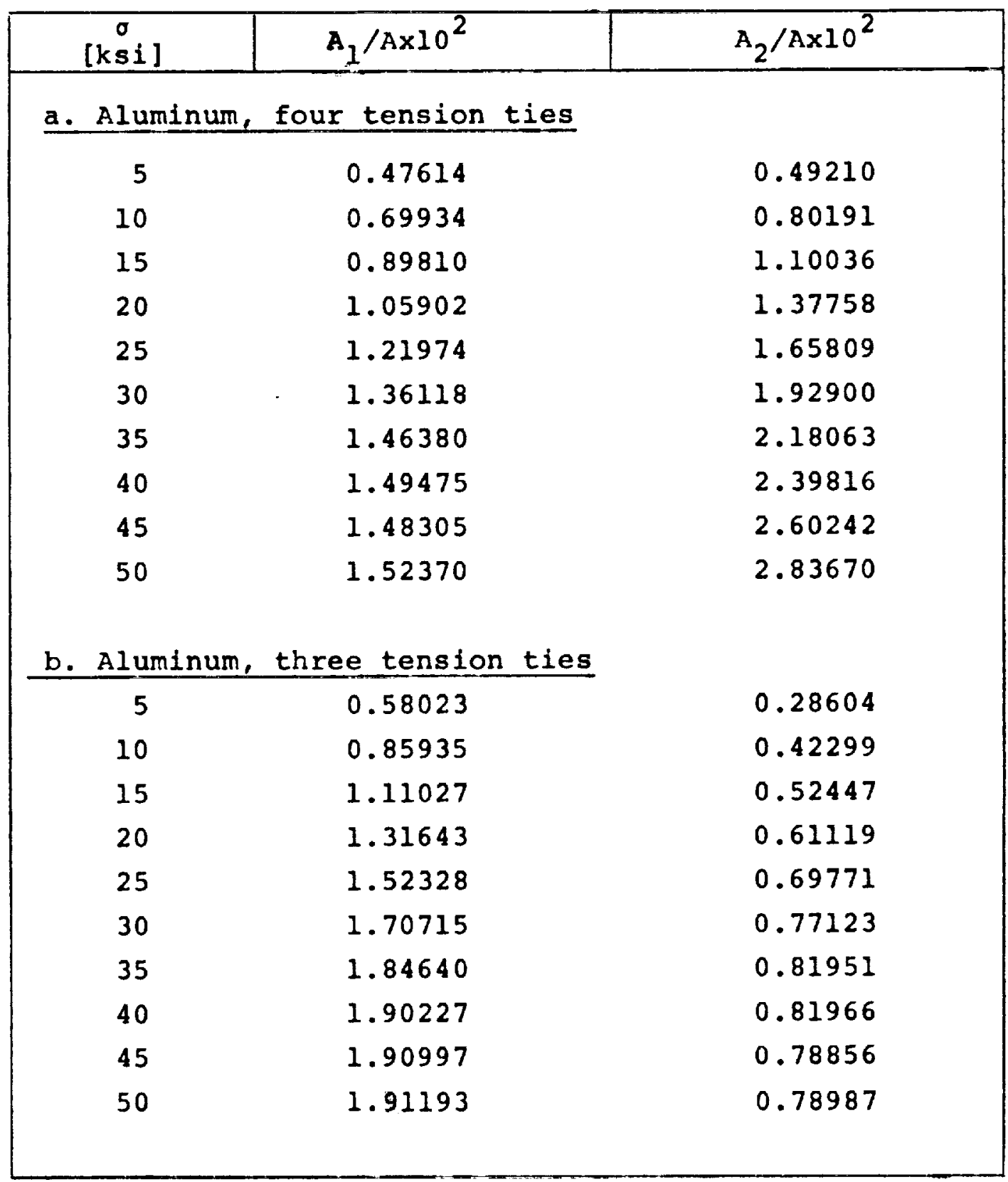


TABLE 6

Cross-sections for a column with three struts, four tension ties, made from steel.

\begin{tabular}{|c|c|c|}
\hline $\begin{array}{c}\sigma \\
{[\mathrm{ksi}]}\end{array}$ & $A_{1} / A \times 10^{2}$ & $A_{2} / A \times 10^{2}$ \\
\hline 5 & 0.28273 & 0.26323 \\
\hline 10 & 0.40425 & 0.40324 \\
\hline 15 & 0.50193 & 0.52712 \\
\hline 20 & 0.58822 & 0.64402 \\
\hline 25 & 0.66732 & 0.74832 \\
\hline 30 & 0.74127 & 0.86311 \\
\hline 35 & 0.81176 & 0.97527 \\
\hline 40 & 0.87901 & 1.08232 \\
\hline 50 & 1.00729 & 1.29371 \\
\hline 60 & 1.11332 & 1.48636 \\
\hline 70 & 1.20580 & 1.68601 \\
\hline 80 & 1.29687 & 1.88009 \\
\hline 90 & 1.38358 & 2.08258 \\
\hline 100 & 1.46535 & 2.26512 \\
\hline 110 & 1.54110 & 2.45446 \\
\hline 120 & 1.59827 & 2.63642 \\
\hline 130 & 1.61218 & 2.79976 \\
\hline 135 & 1.50938 & 2.83423 \\
\hline
\end{tabular}


TABLE 7

Cross-sections for a column with three struts, three tension ties, made from steel.

\begin{tabular}{|c|c|c|}
\hline $\begin{array}{c}\sigma \\
{[k s i]}\end{array}$ & $A_{1} / A \times 10^{2}$ & $A_{2} / A \times 10^{2}$ \\
\hline 5 & 0.34190 & 0.17257 \\
\hline 10 & 0.49116 & 0.24423 \\
\hline 15 & 0.61226 & 0.30337 \\
\hline 20 & 0.72005 & 0.35022 \\
\hline 25 & 0.81920 & 0.39497 \\
\hline 30 & 0.91945 & 0.43648 \\
\hline 35 & 1.00146 & 0.47557 \\
\hline 40 & 1.08718 & 0.51278 \\
\hline 50 & 1.25084 & 0.58295 \\
\hline 60 & 1.38845 & 0.63868 \\
\hline 70 & 1.51025 & 0.68539 \\
\hline 80 & 1.63065 & 0.73089 \\
\hline 90 & 1.74650 & 0.77342 \\
\hline 100 & 1.85632 & 0.81226 \\
\hline 110 & 1.95833 & 0.84622 \\
\hline 120 & 2.04084 & 0.86828 \\
\hline 130 & 2.07327 & 0.86065 \\
\hline 135 & 1.96292 & 0.77879 \\
\hline
\end{tabular}




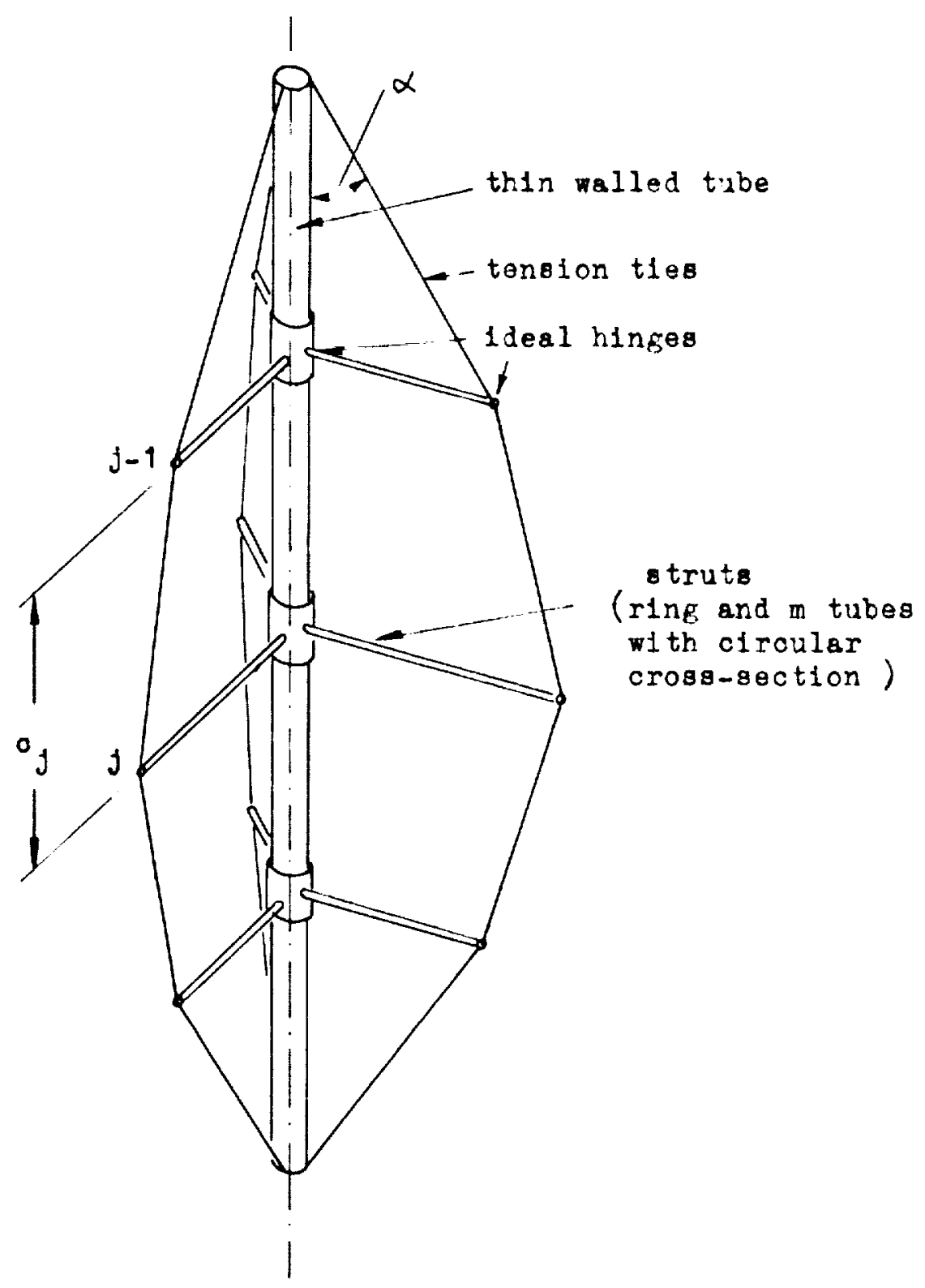

FIG.I: Structural parts of the column supported by tension t1es. 


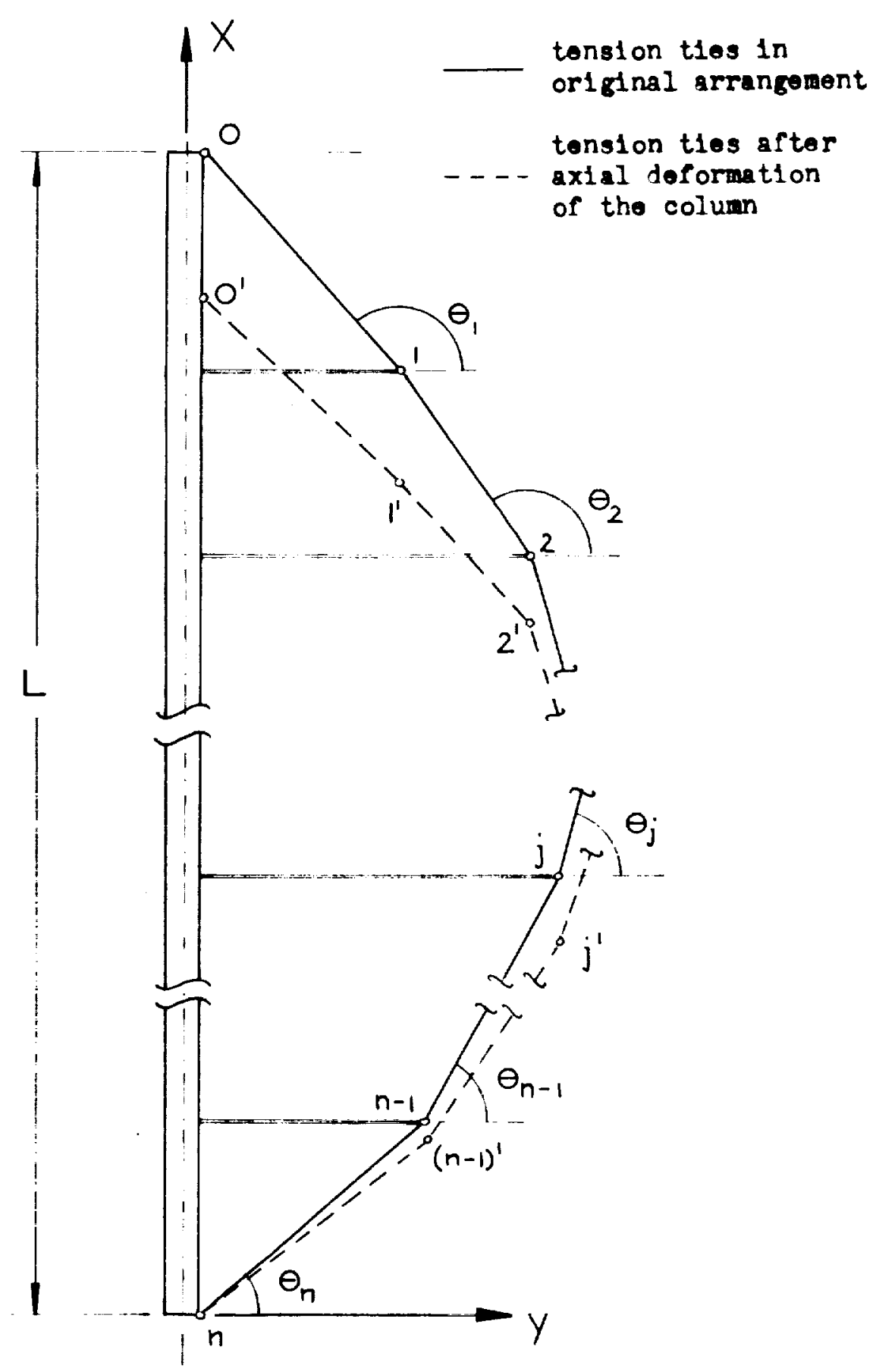

FIG. 2: Dometry of the wro supportod column whth n-1 struts. 


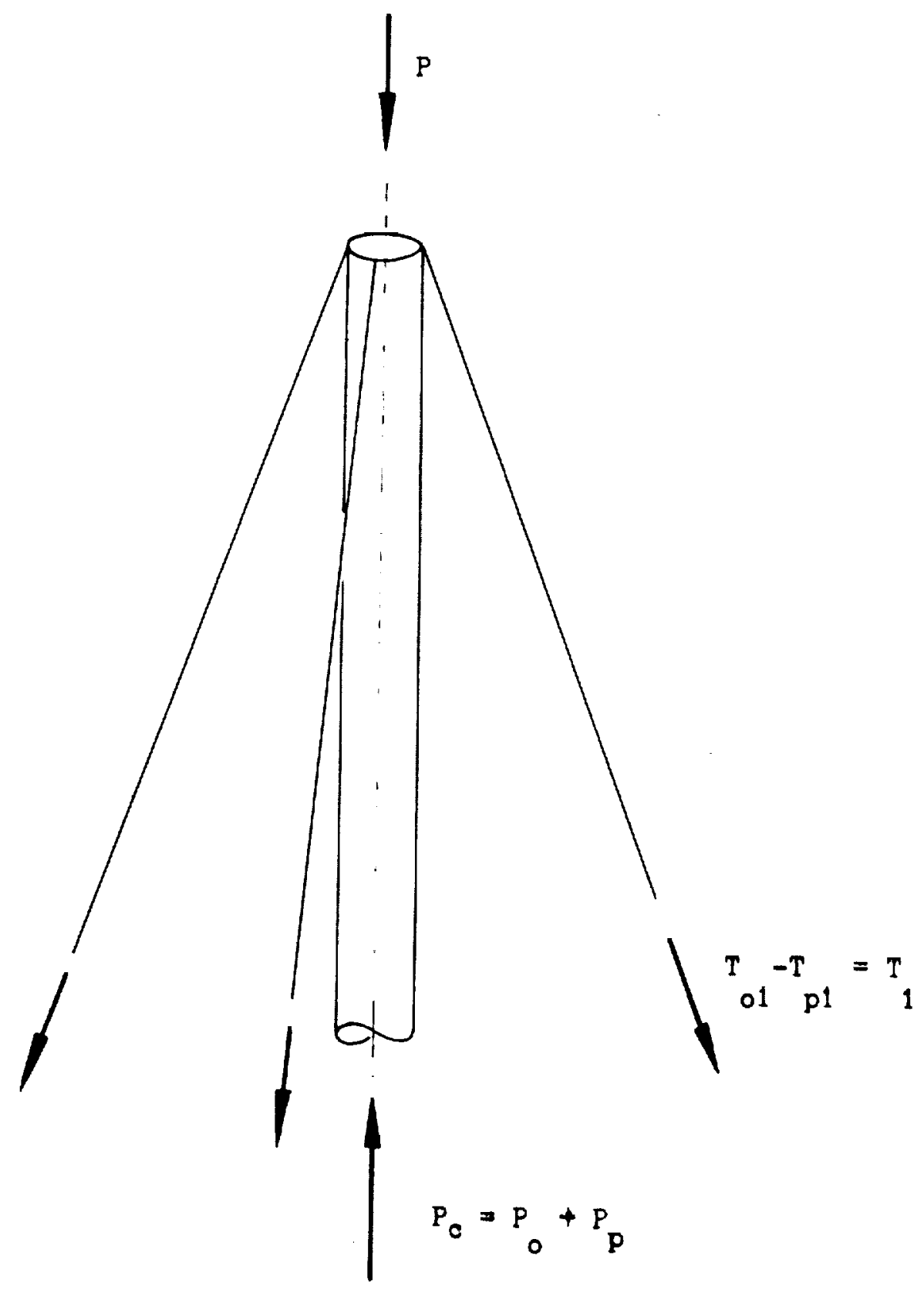

FIG.3: Equilibrium of forces at the end of the colum $(x=L)$. 


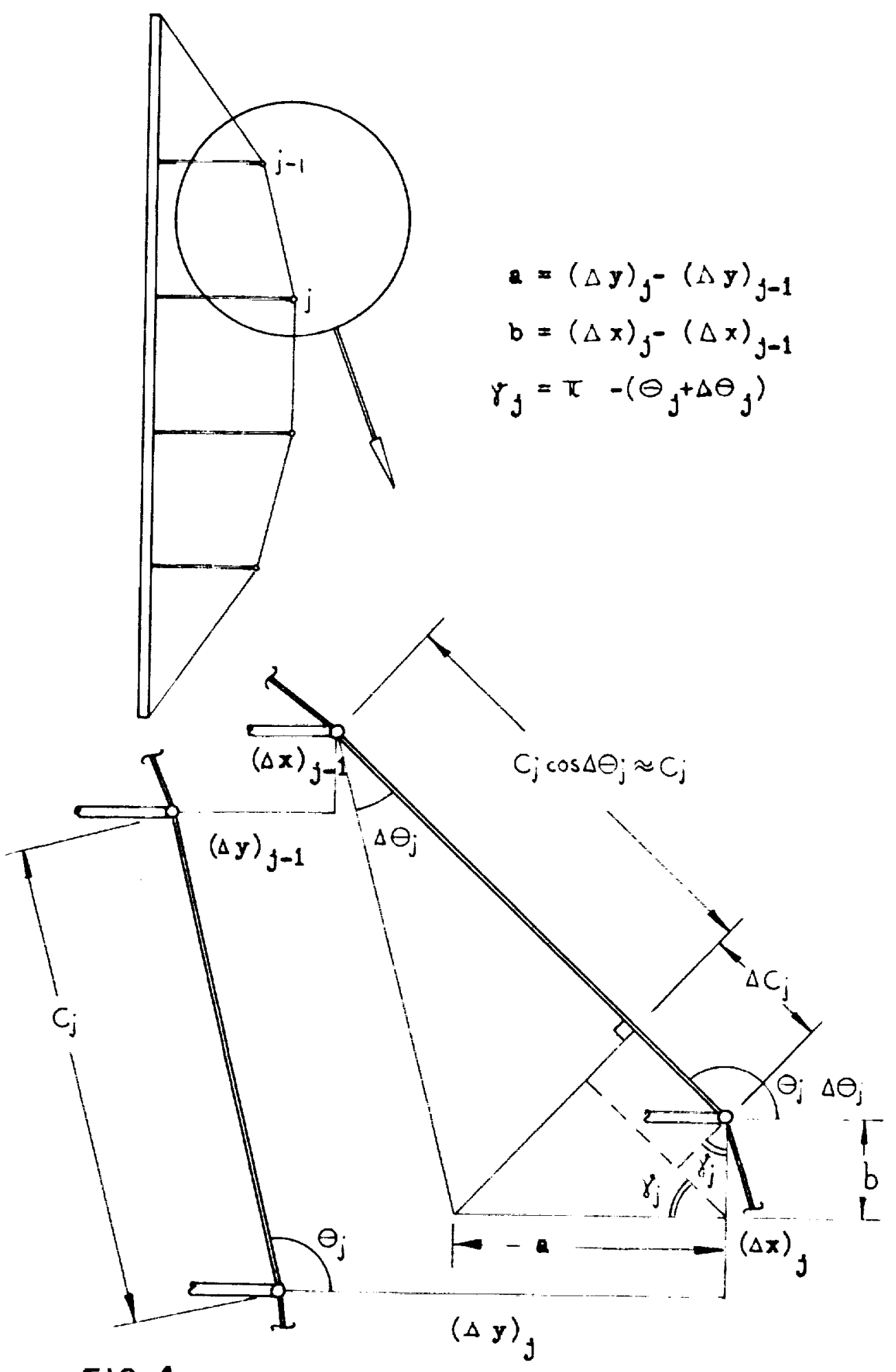

FIG. 4: Geowetry of tie $\mathrm{J}$ before and after lateral deflection. 


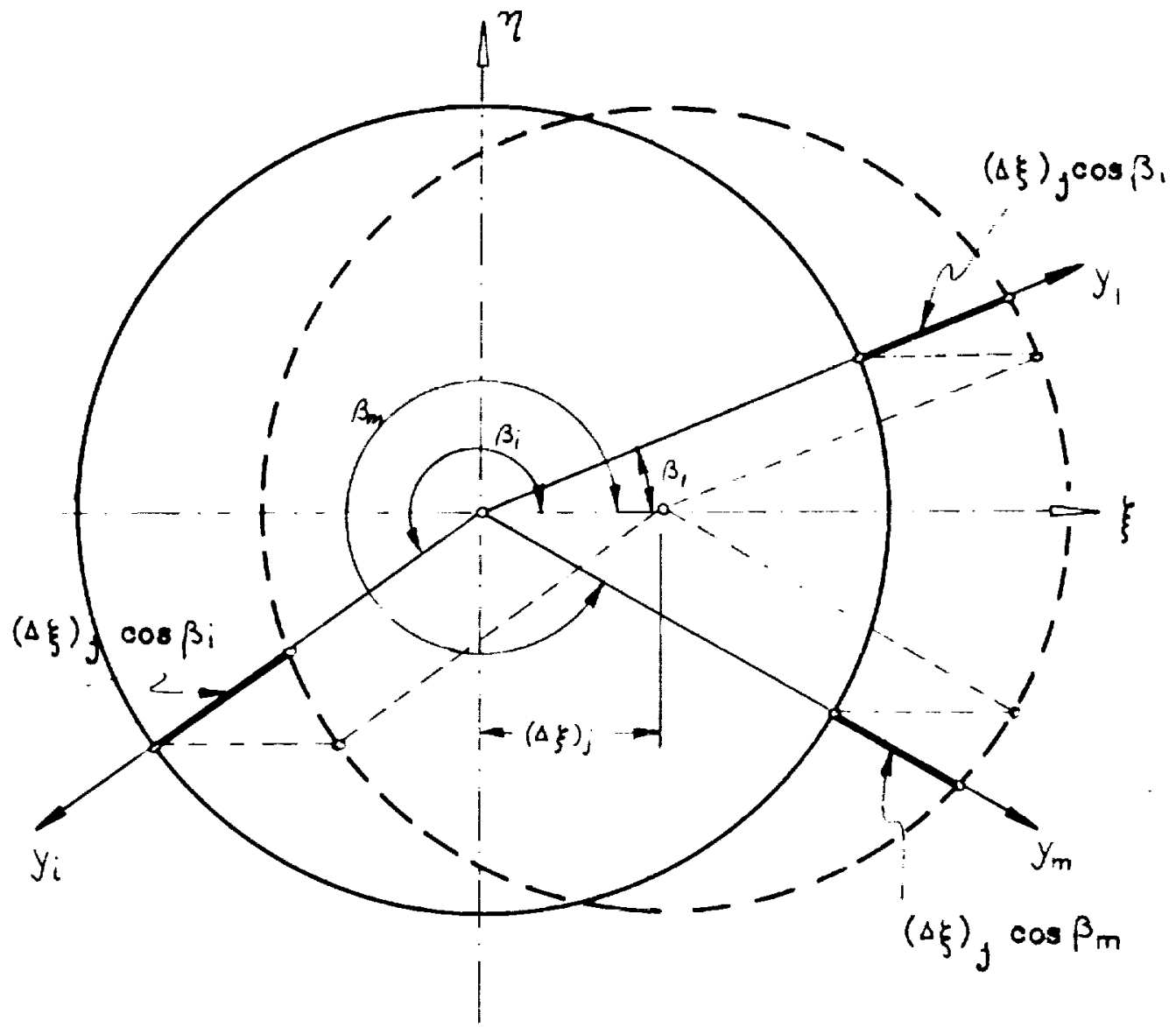

strut plane I before deflection

- $\ldots$ strut plane $j$ after deflection $(\Delta \xi)_{j}$

FIG.5: Deflection geometry at the plane of strut $f$. 


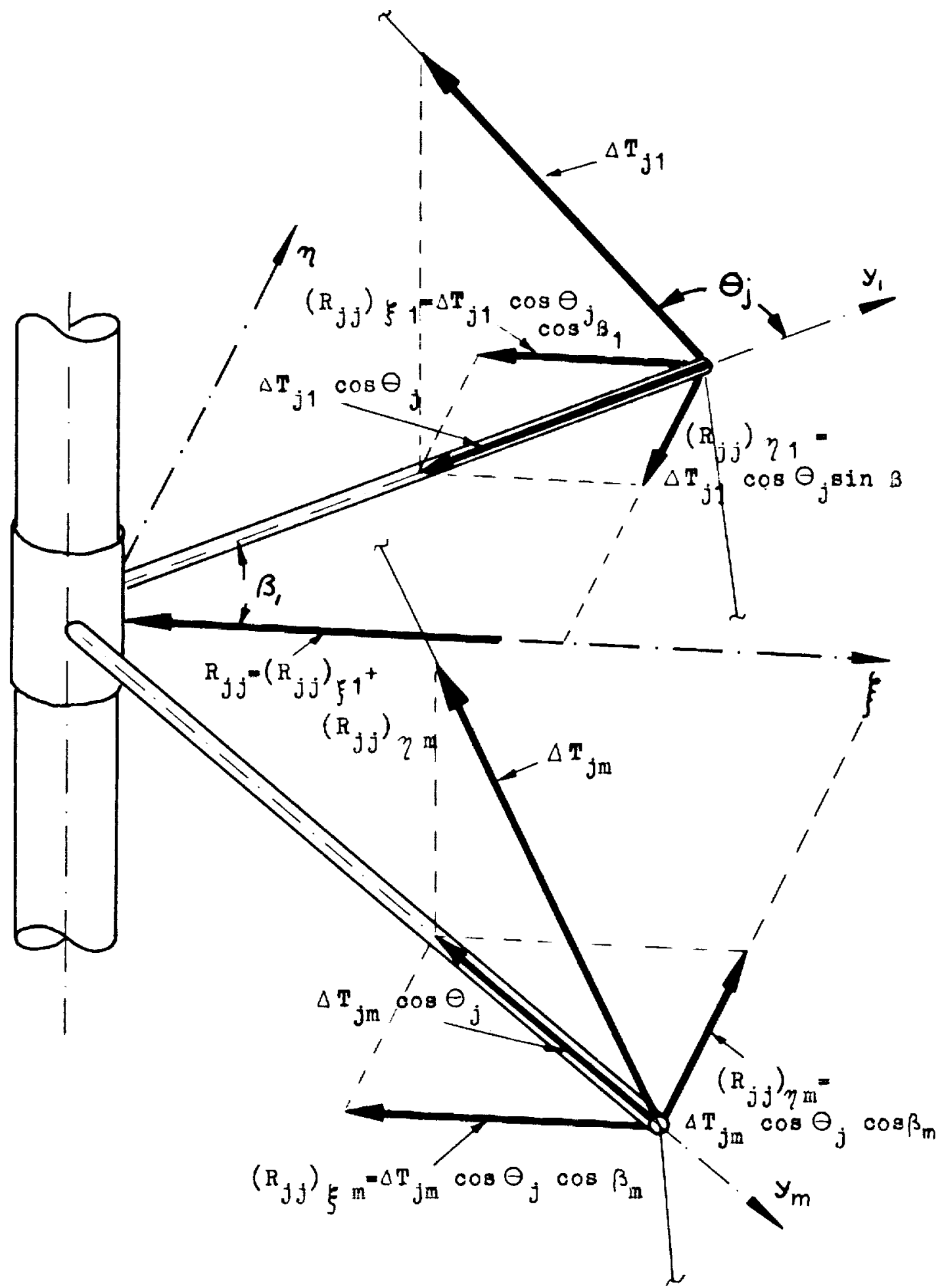

FIG.6, Components of tensile forces in direction $\xi$ in the strut panel $j$ 。 

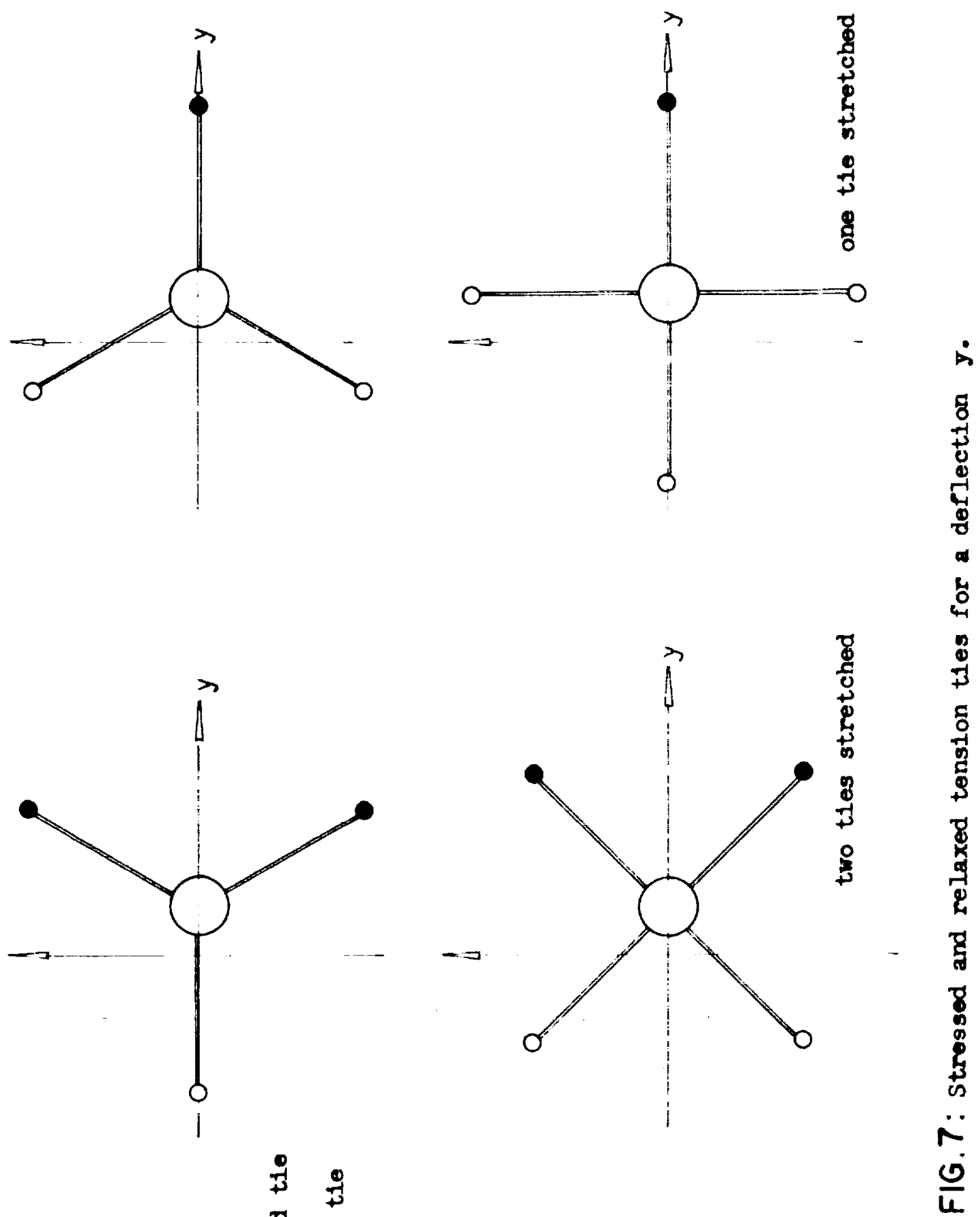

$\sim$

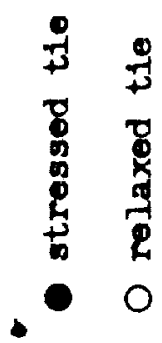




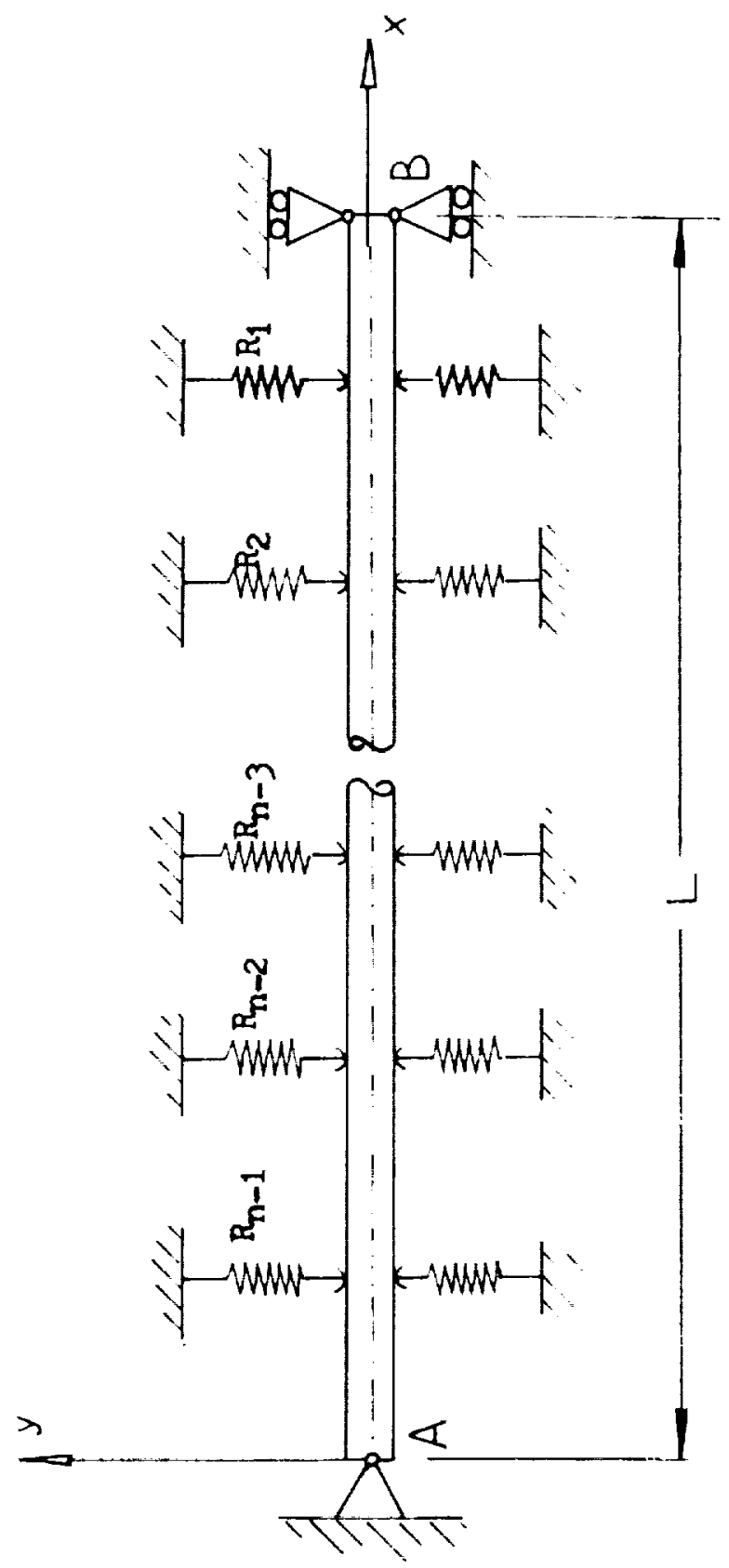

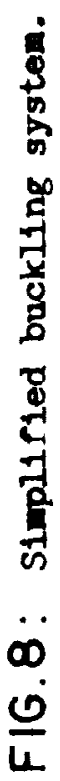




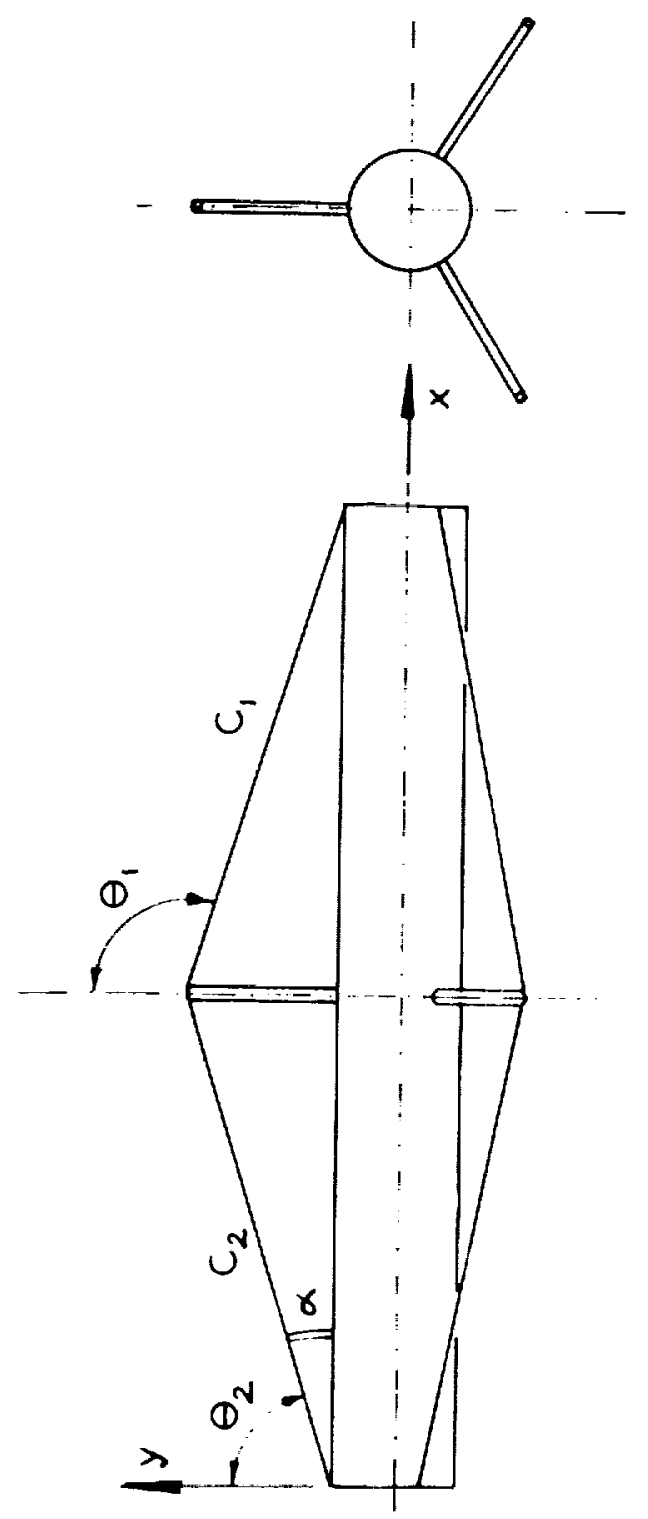

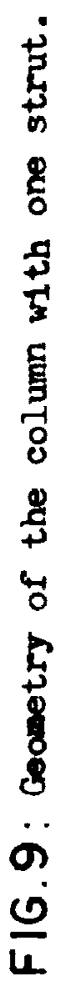




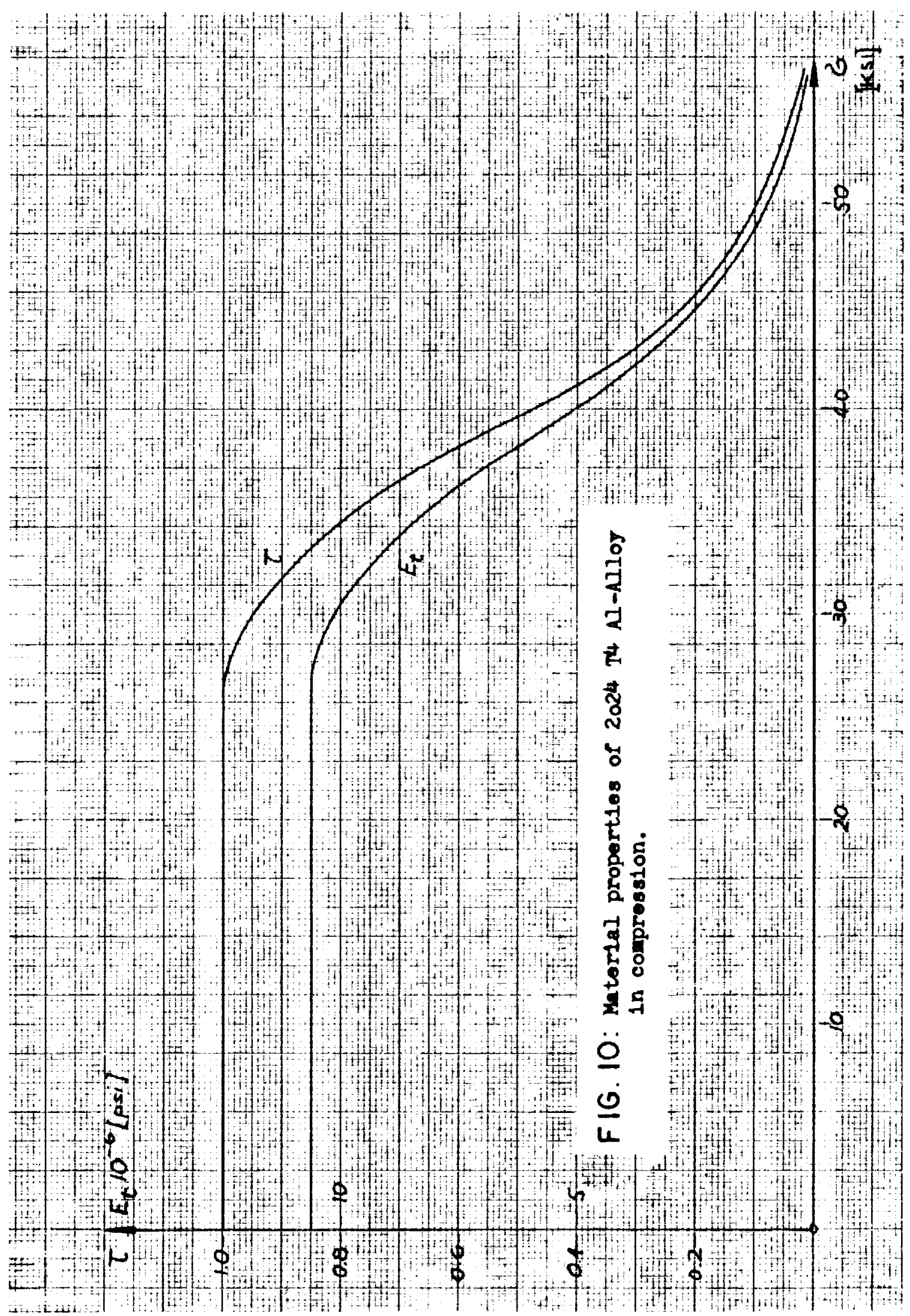




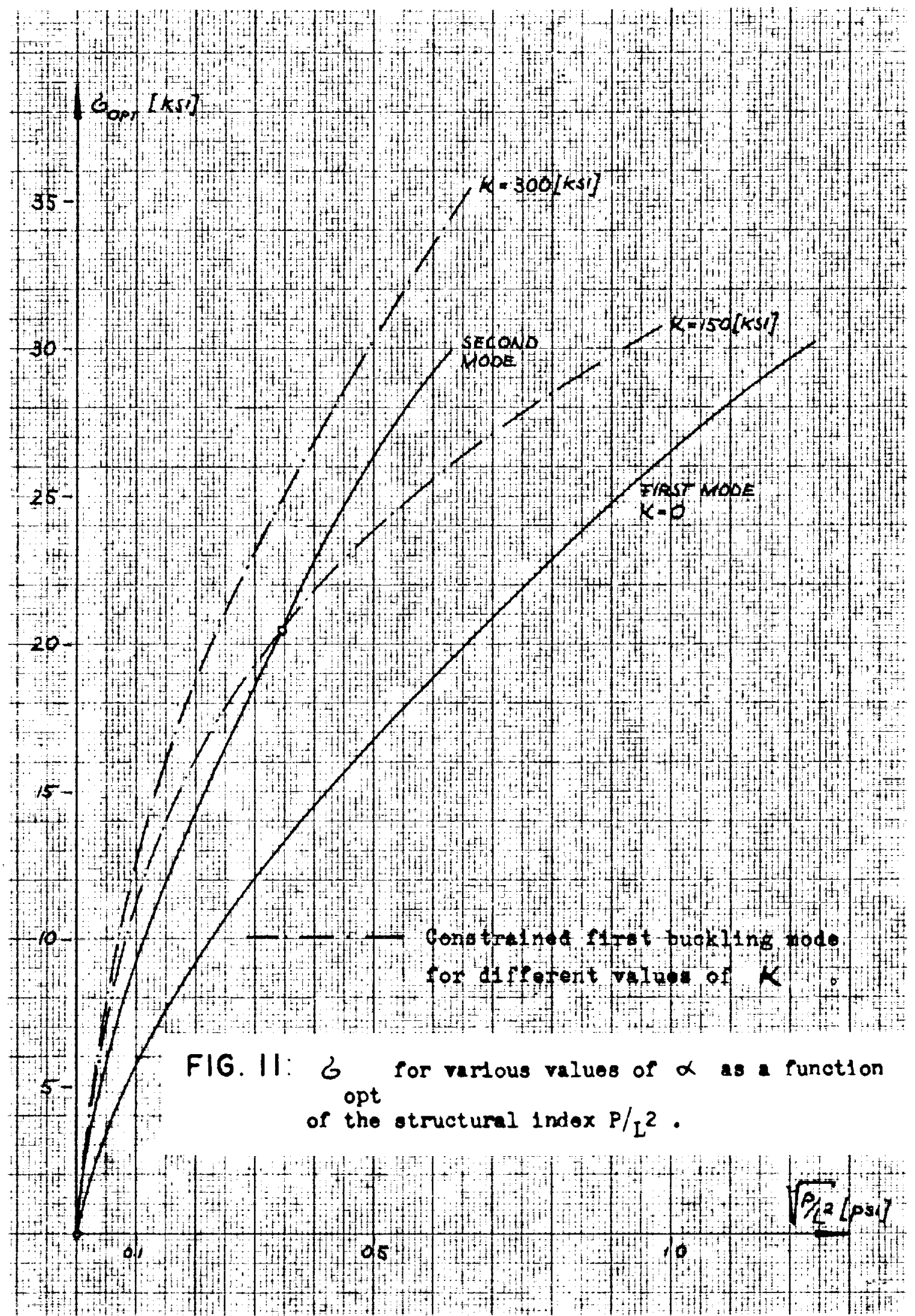



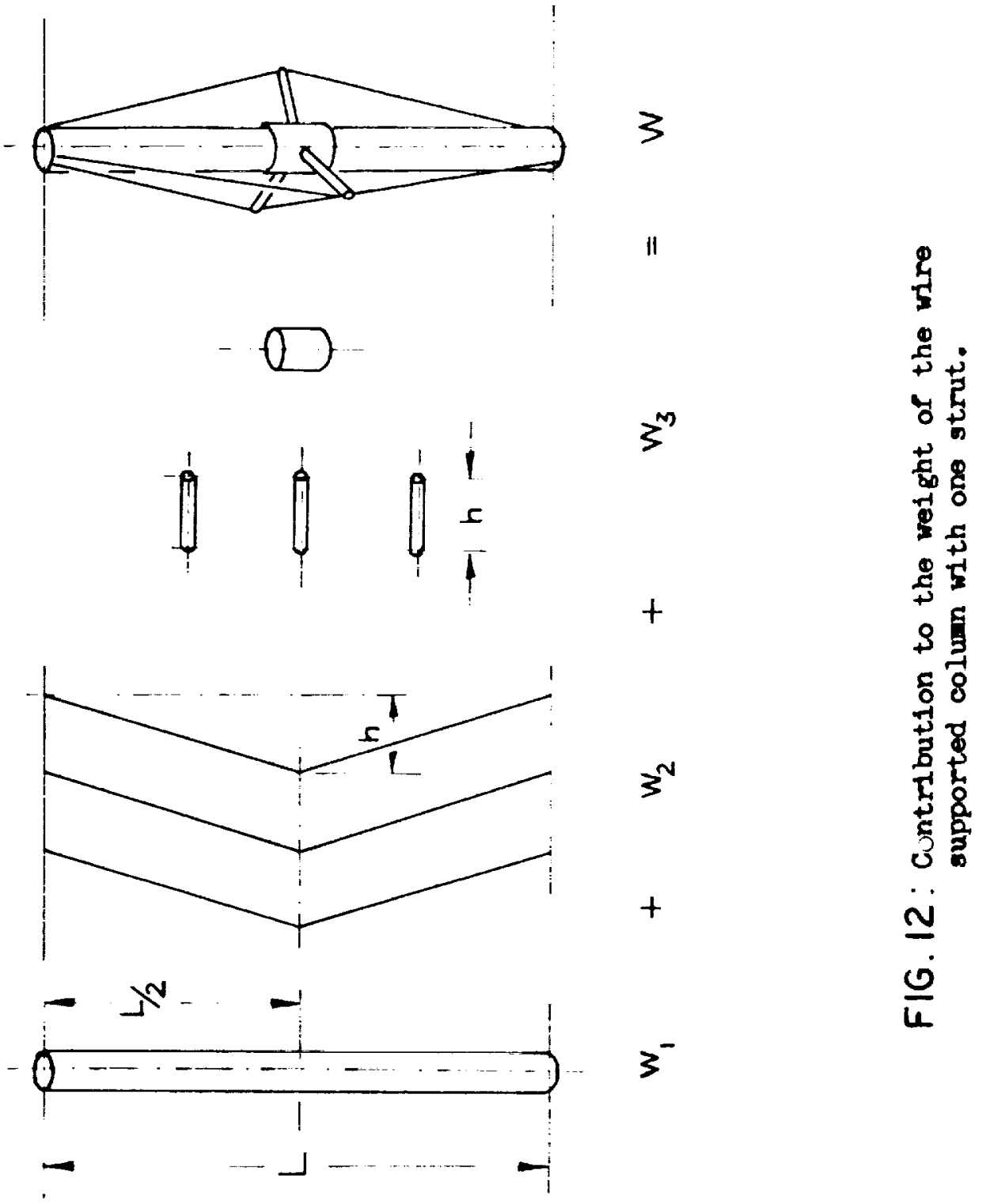


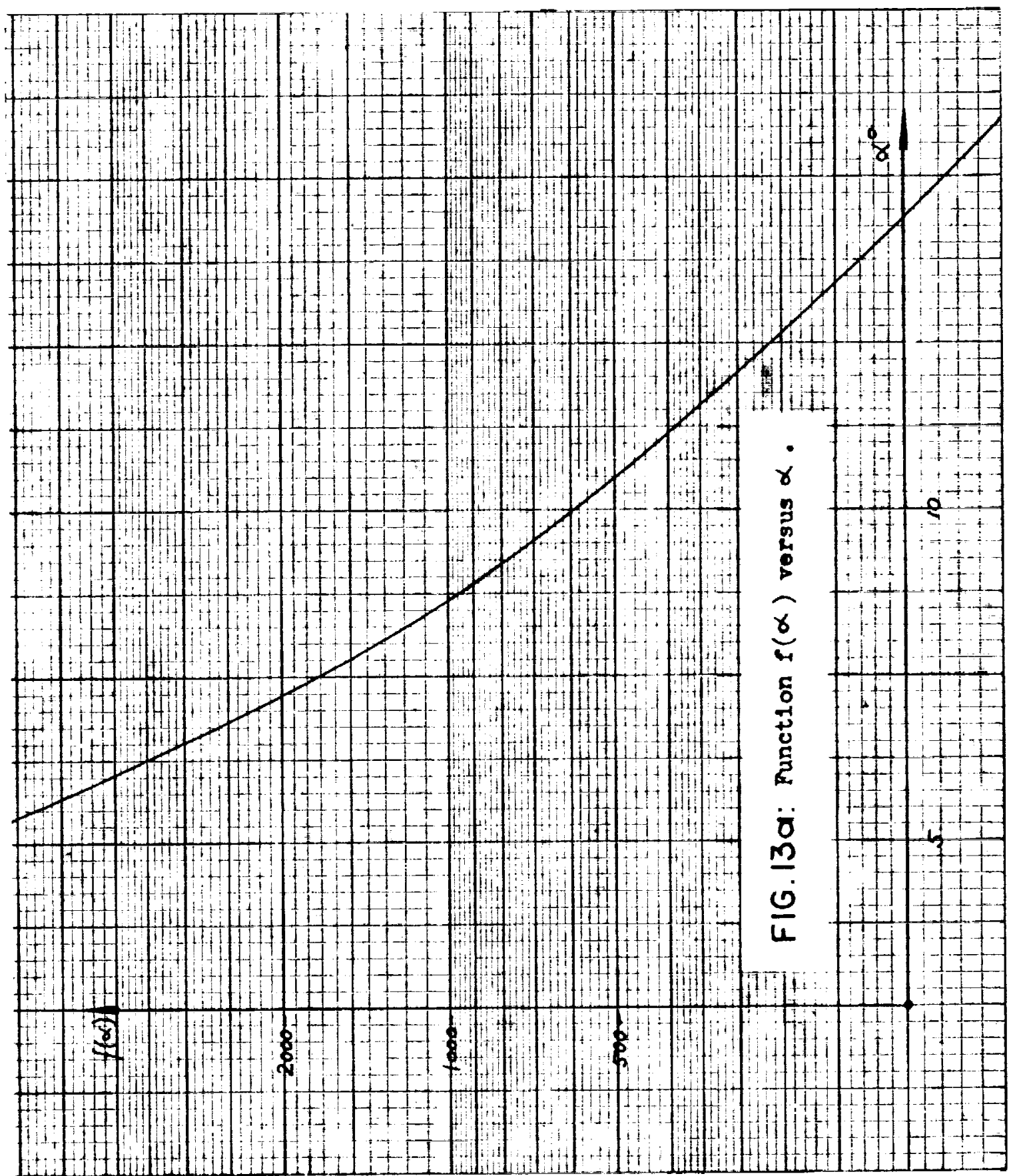




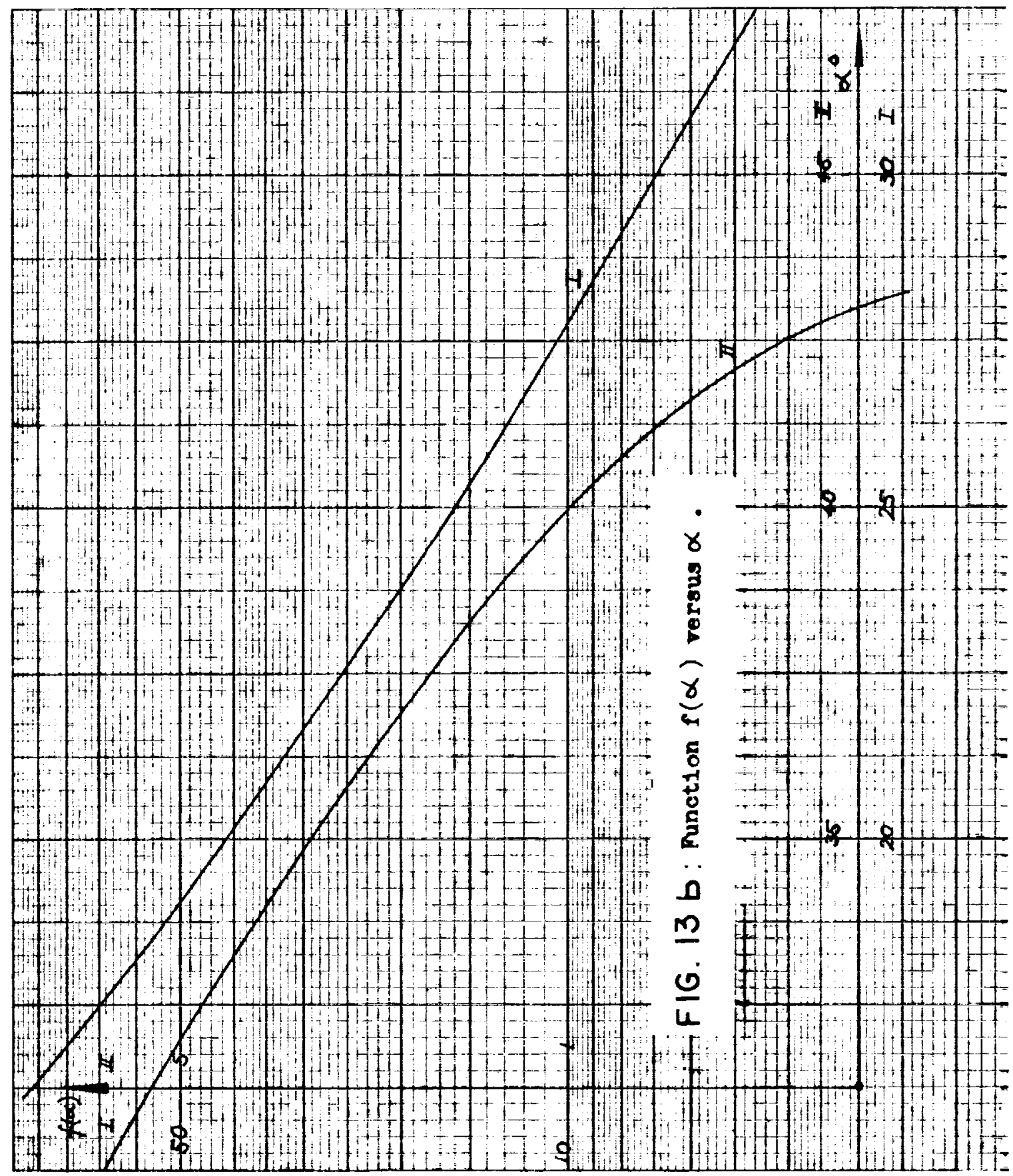



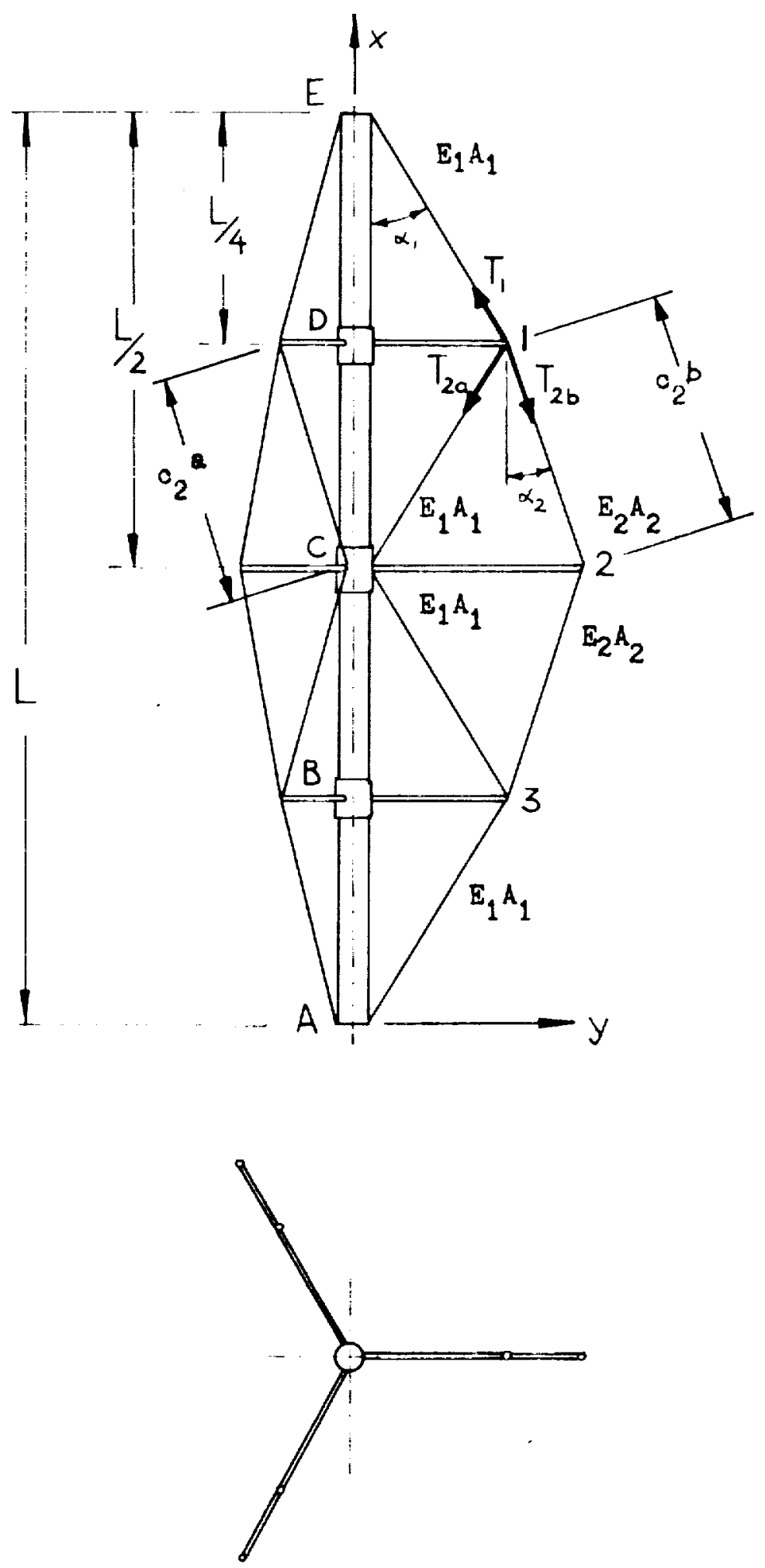

F IG. 14: Coometry of the system with three struts. 

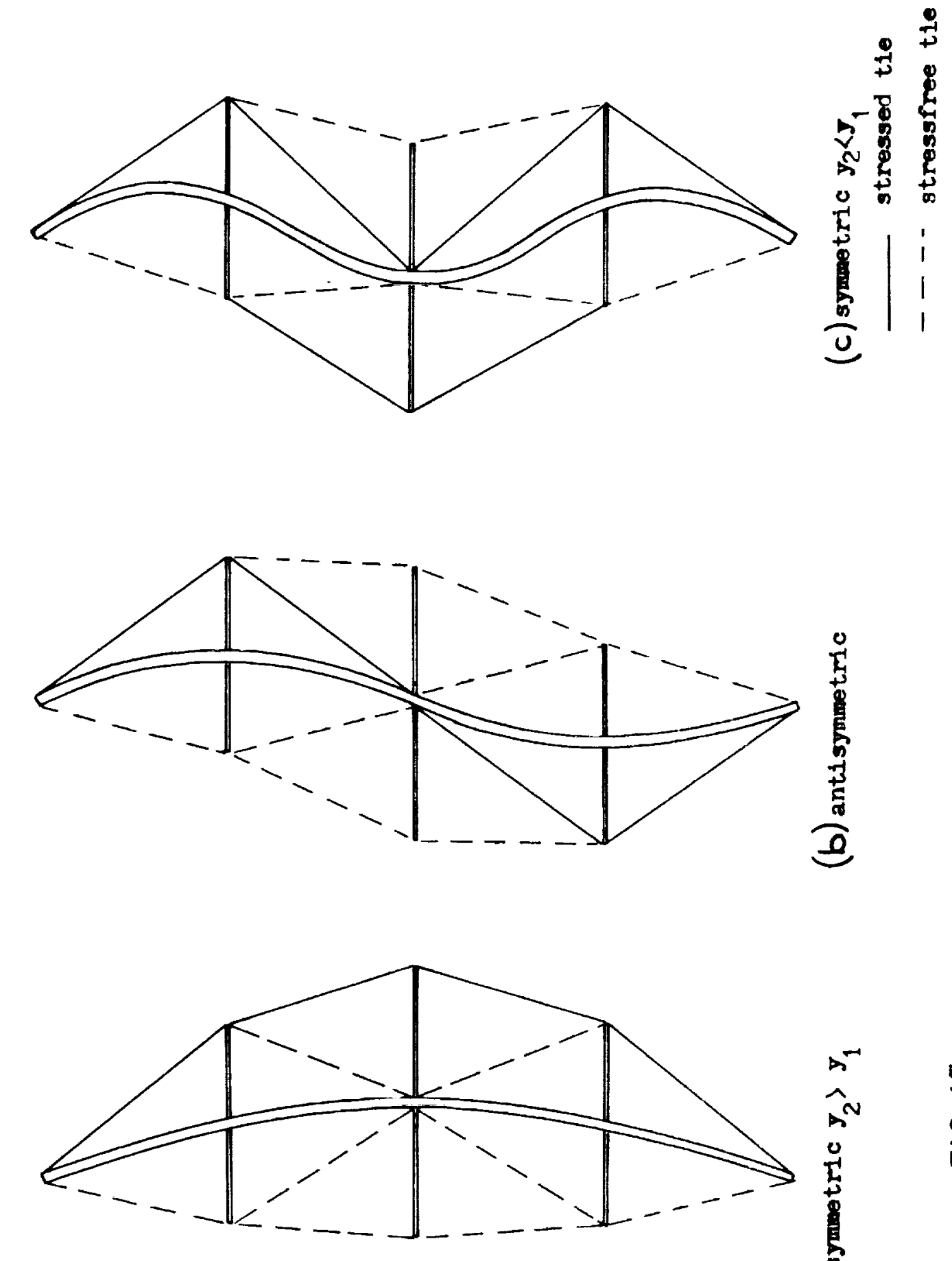

อ

章

車
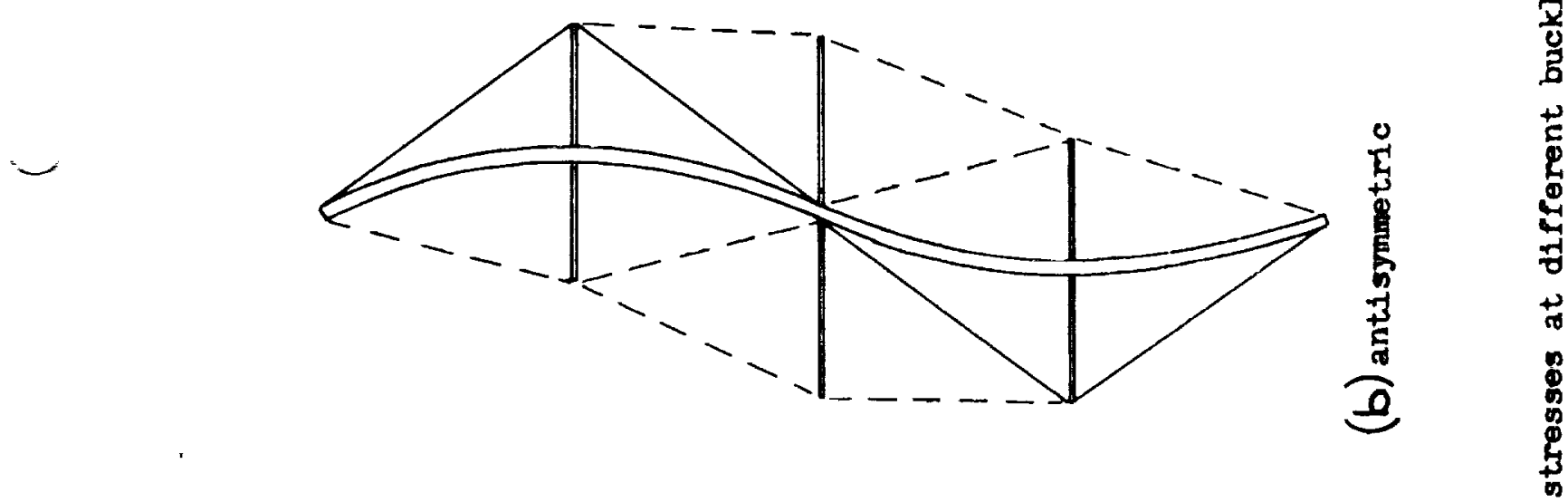

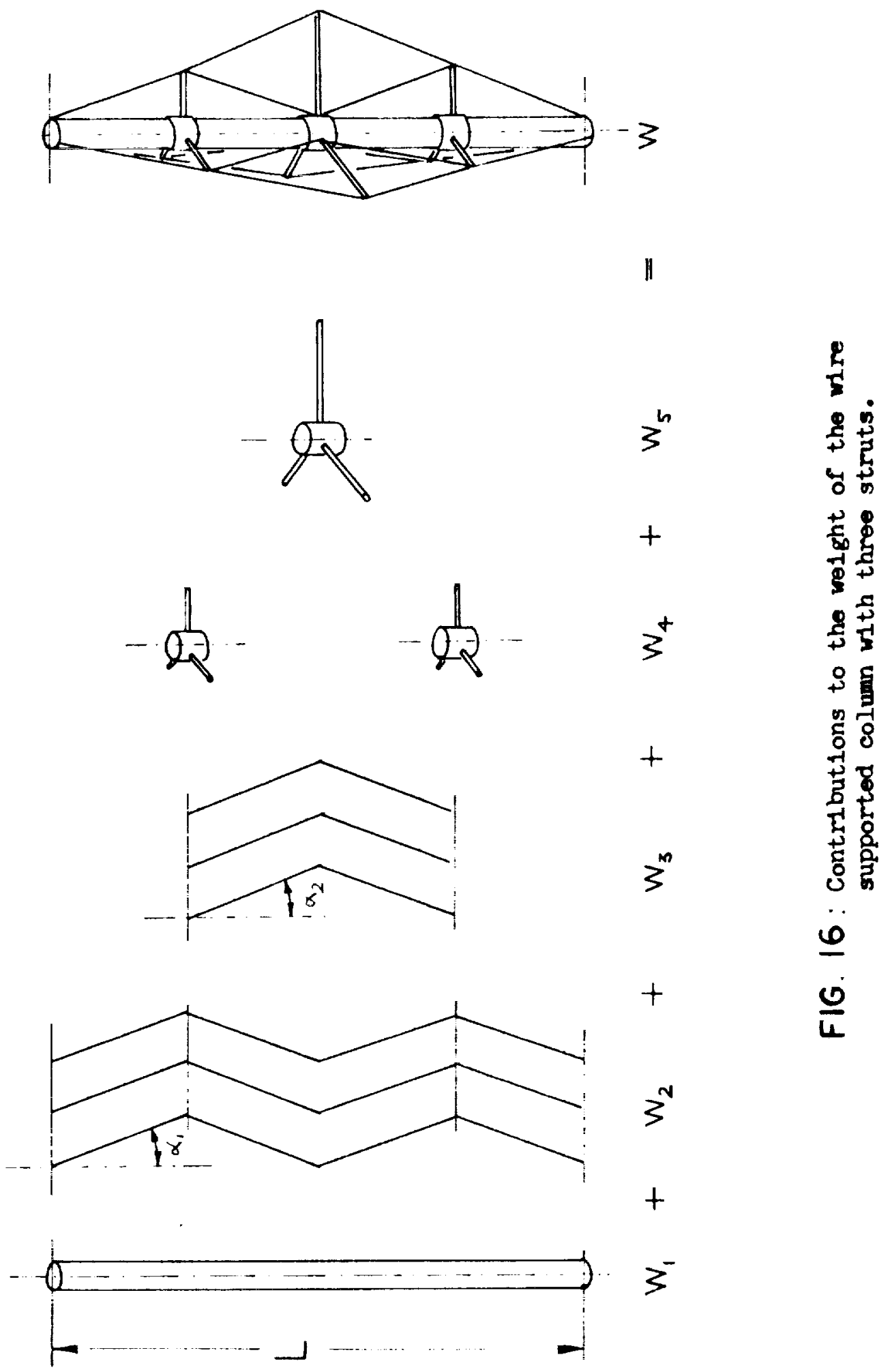


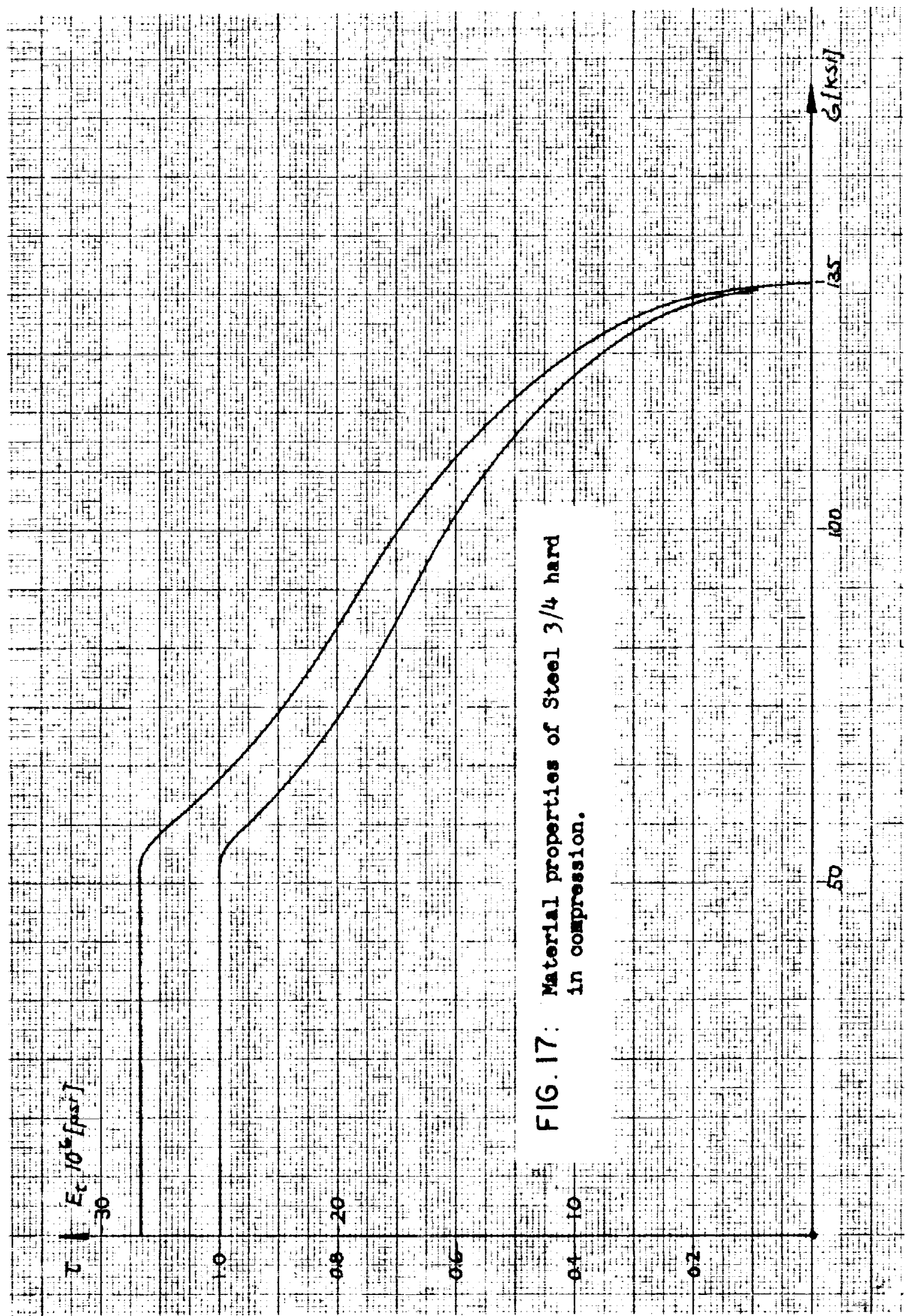




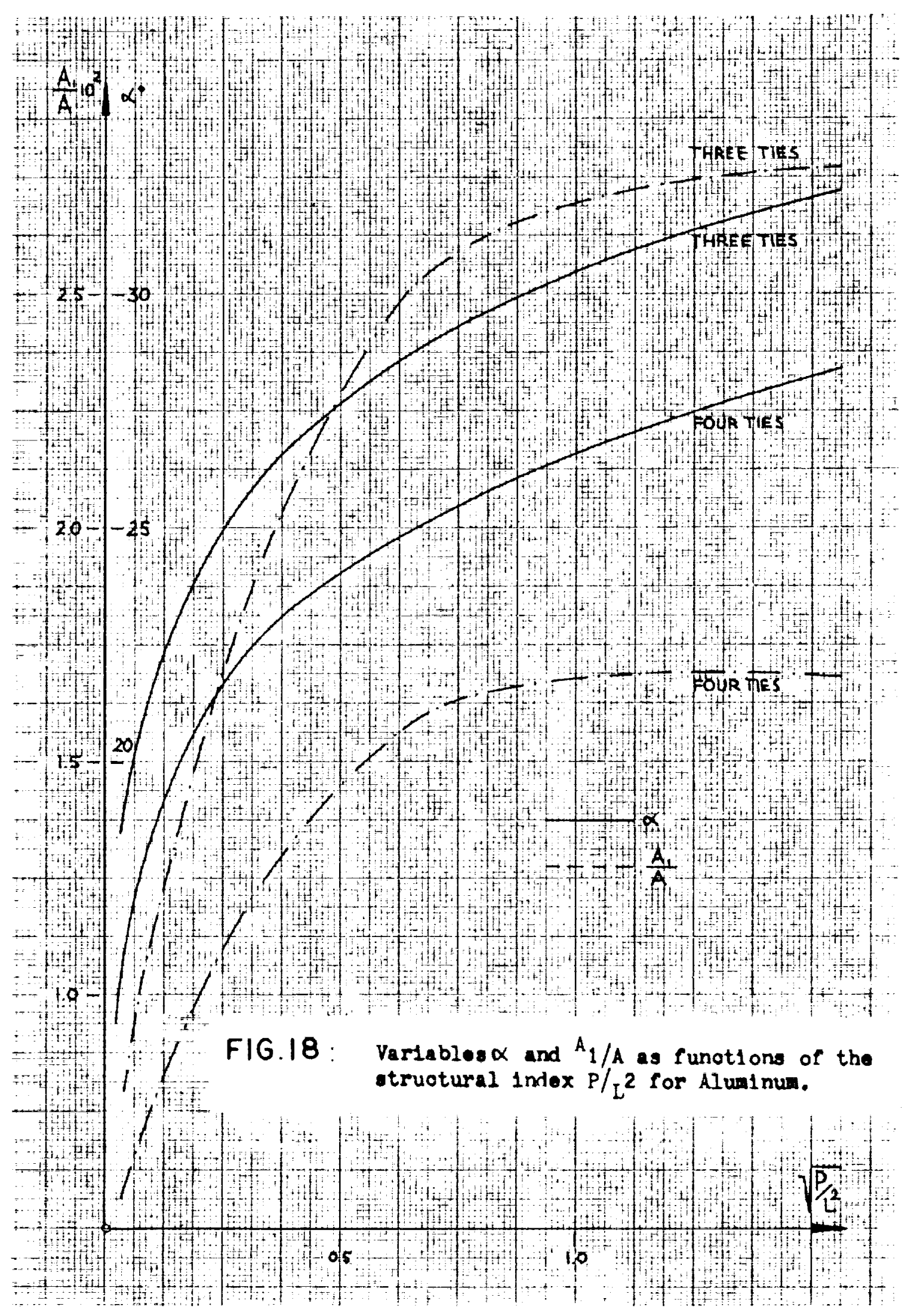




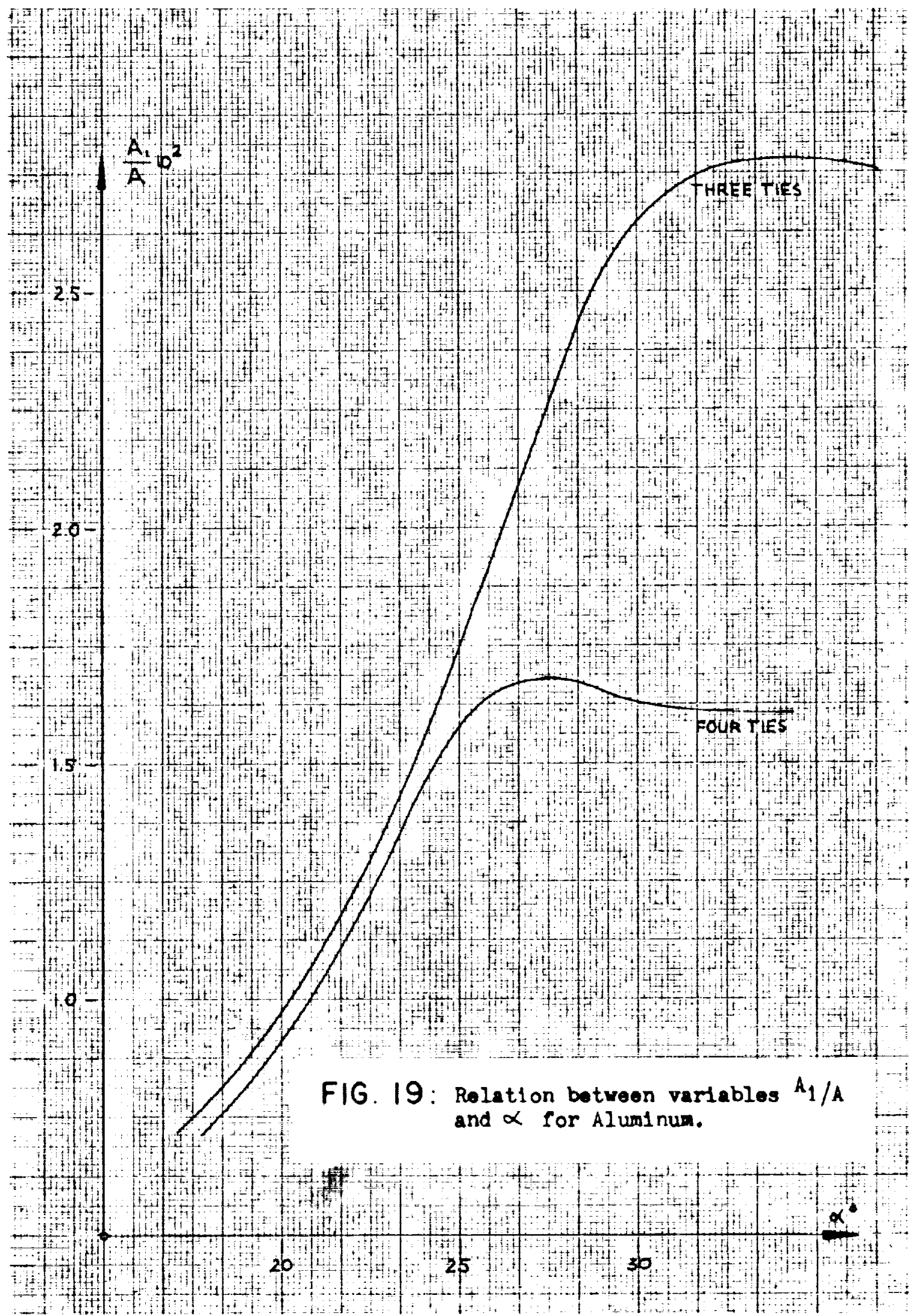




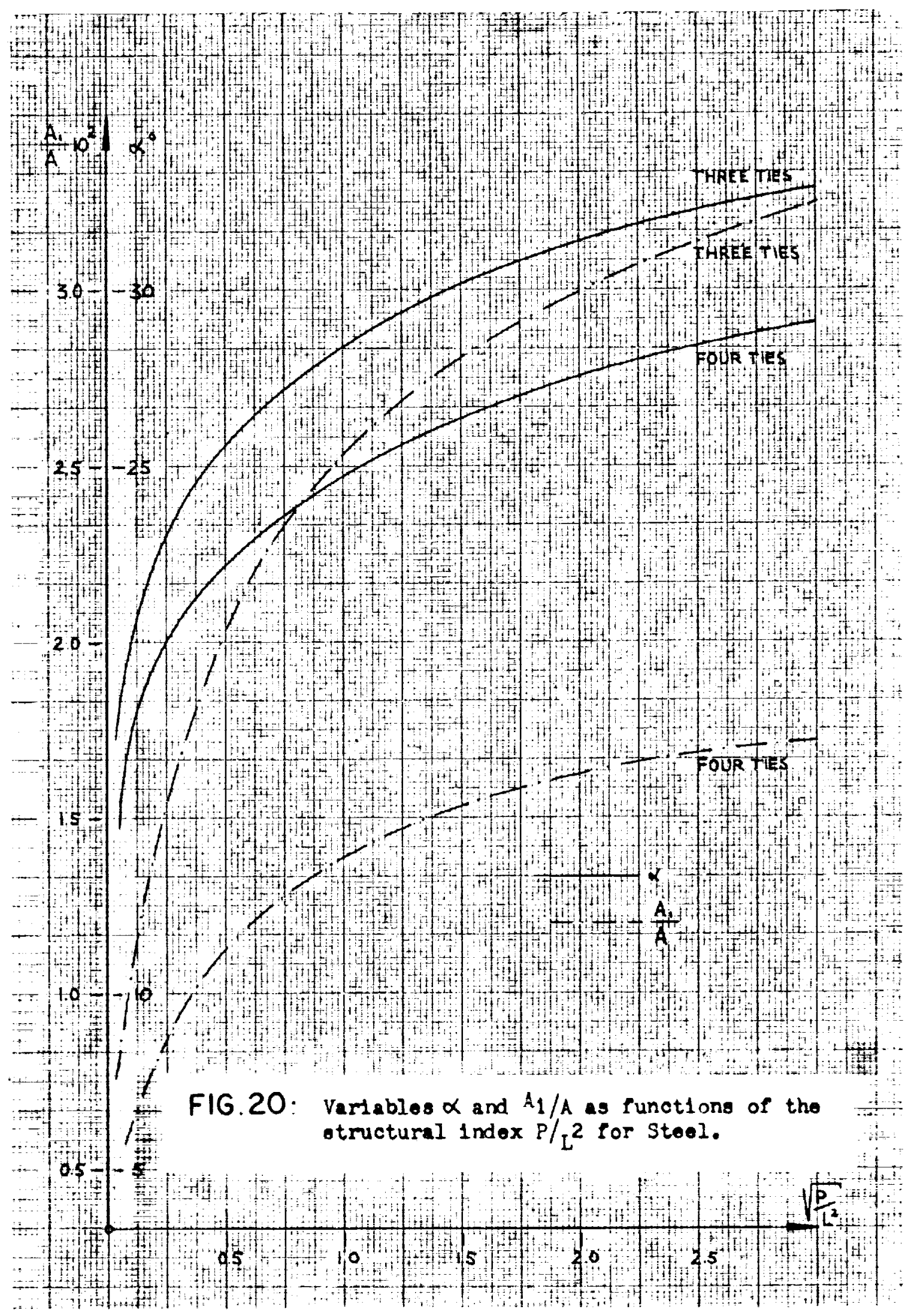




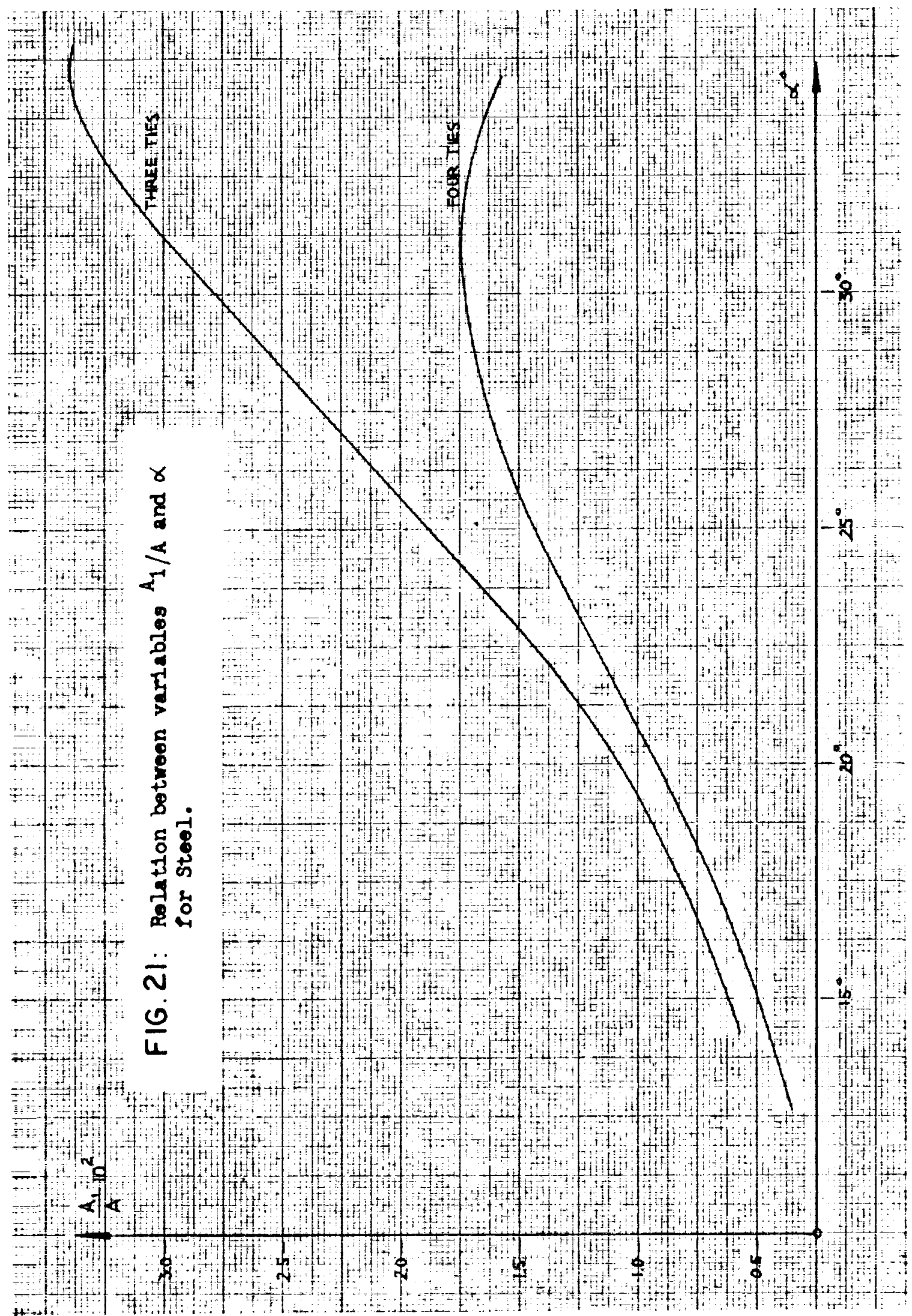




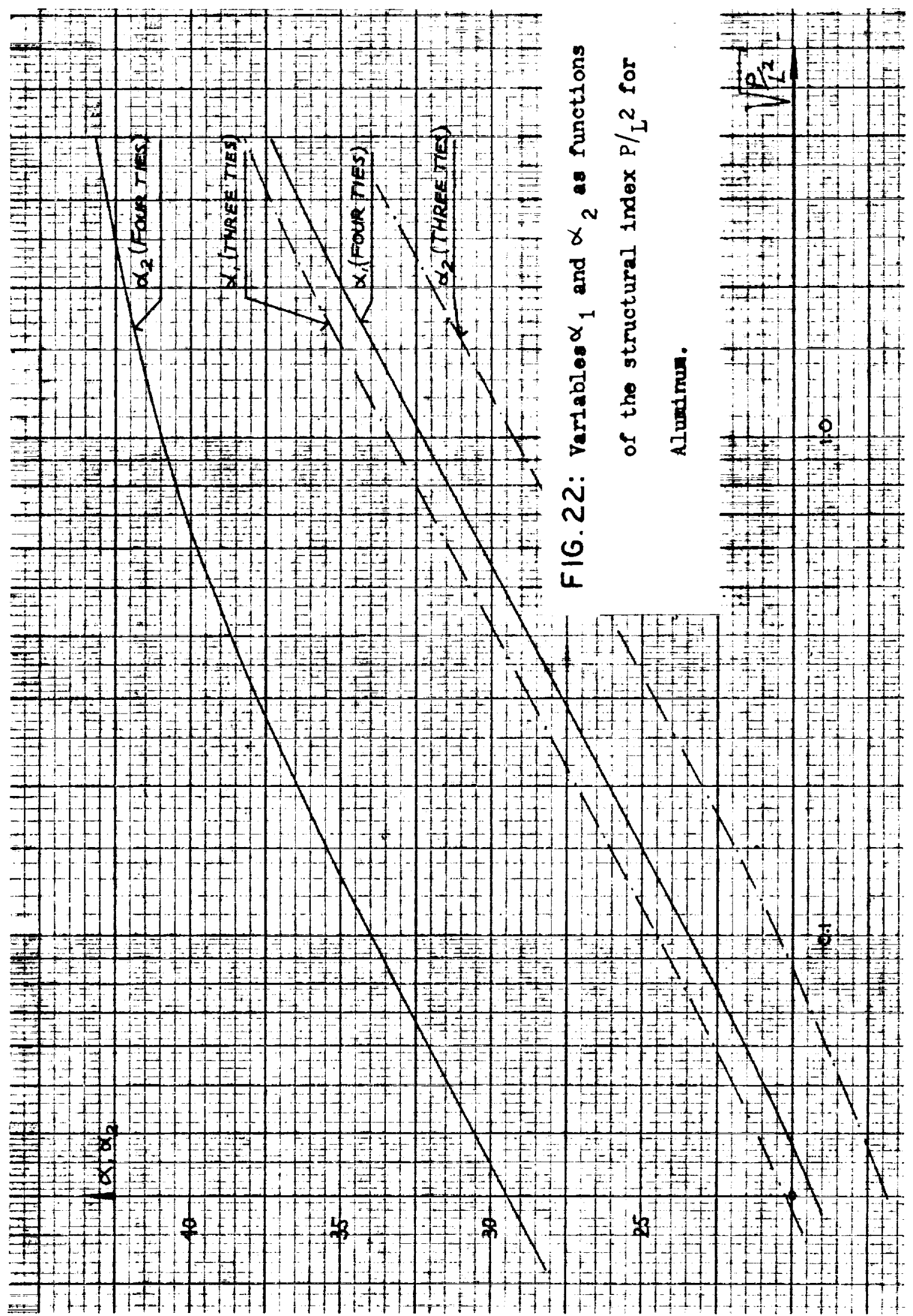




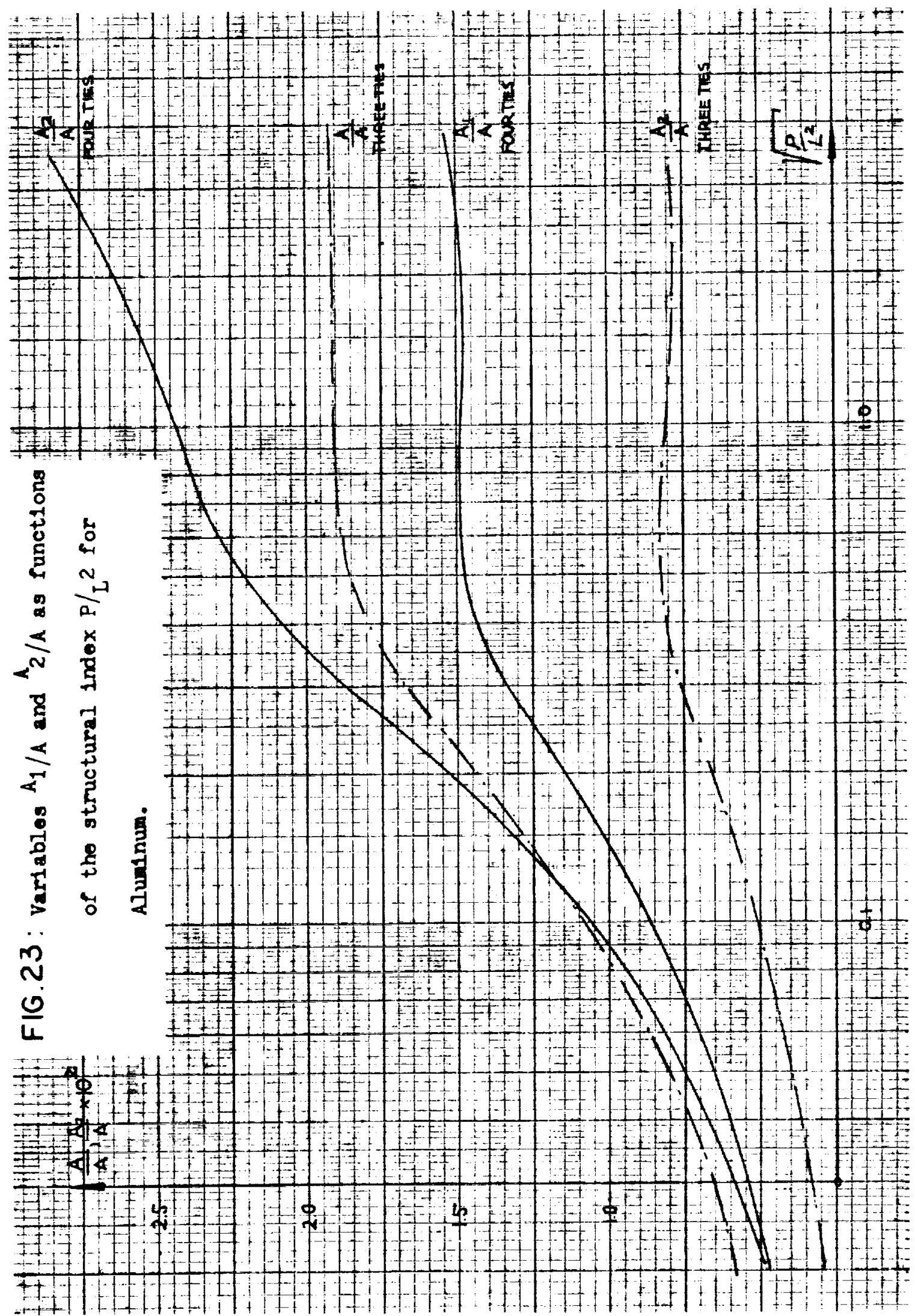




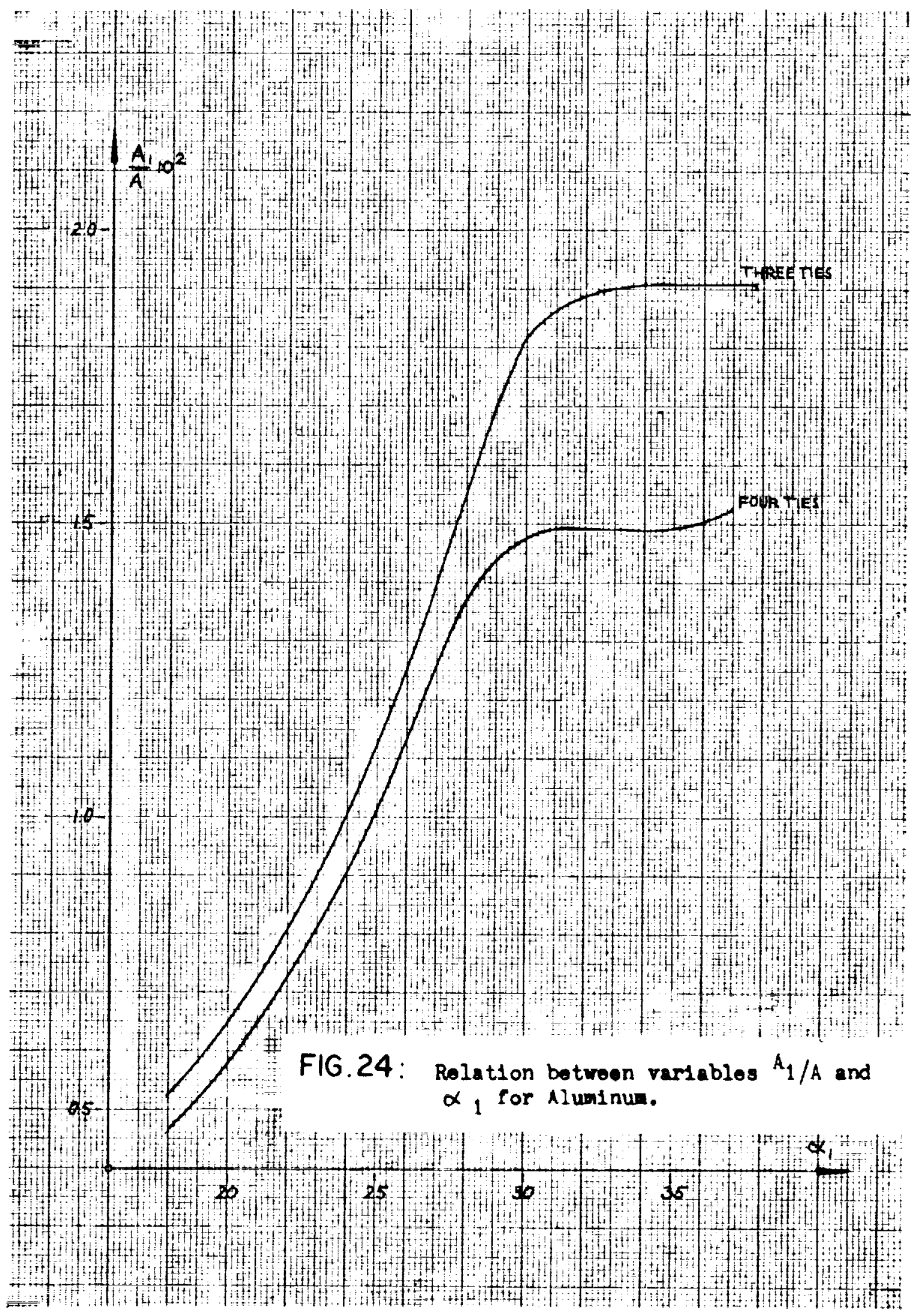




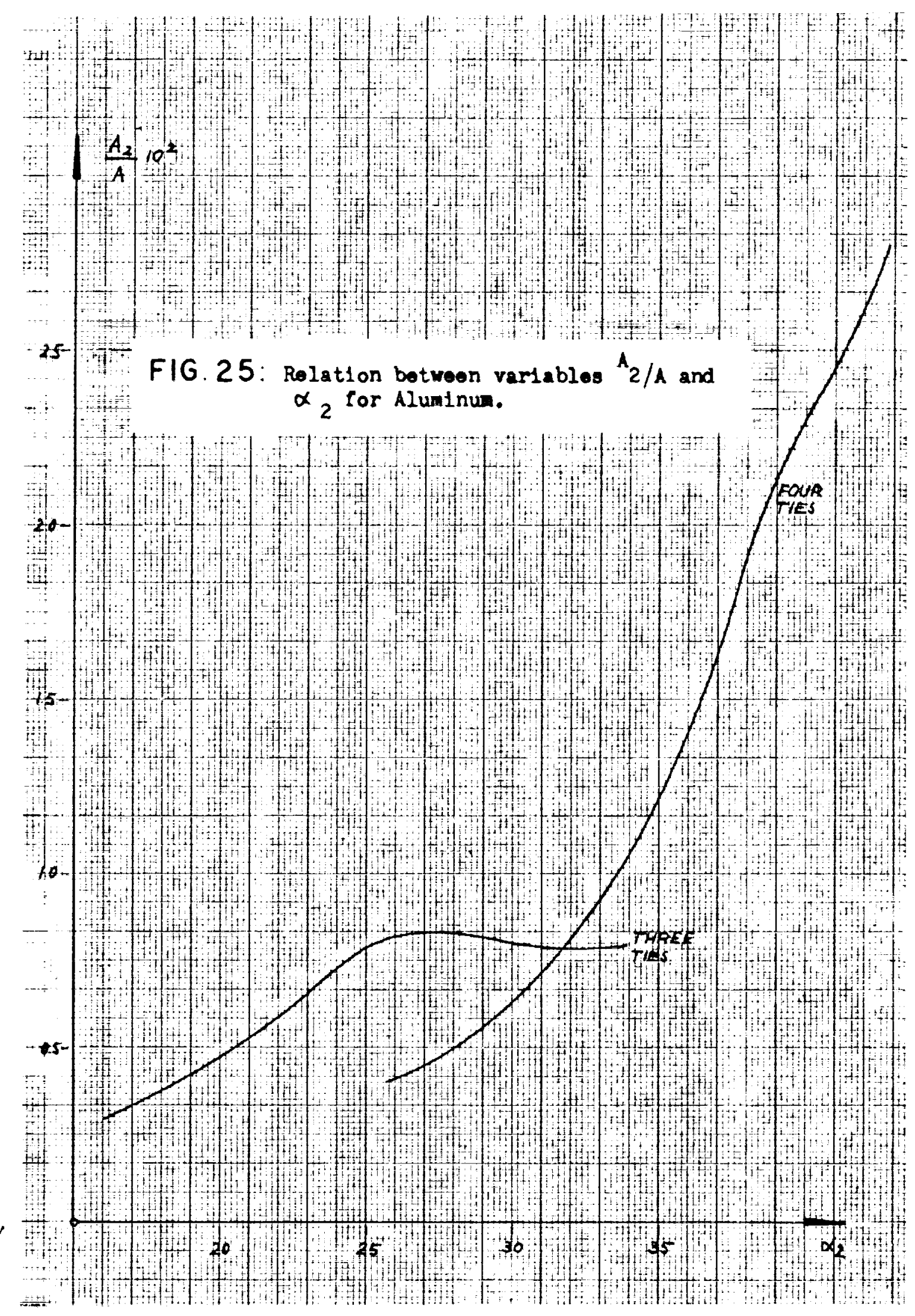




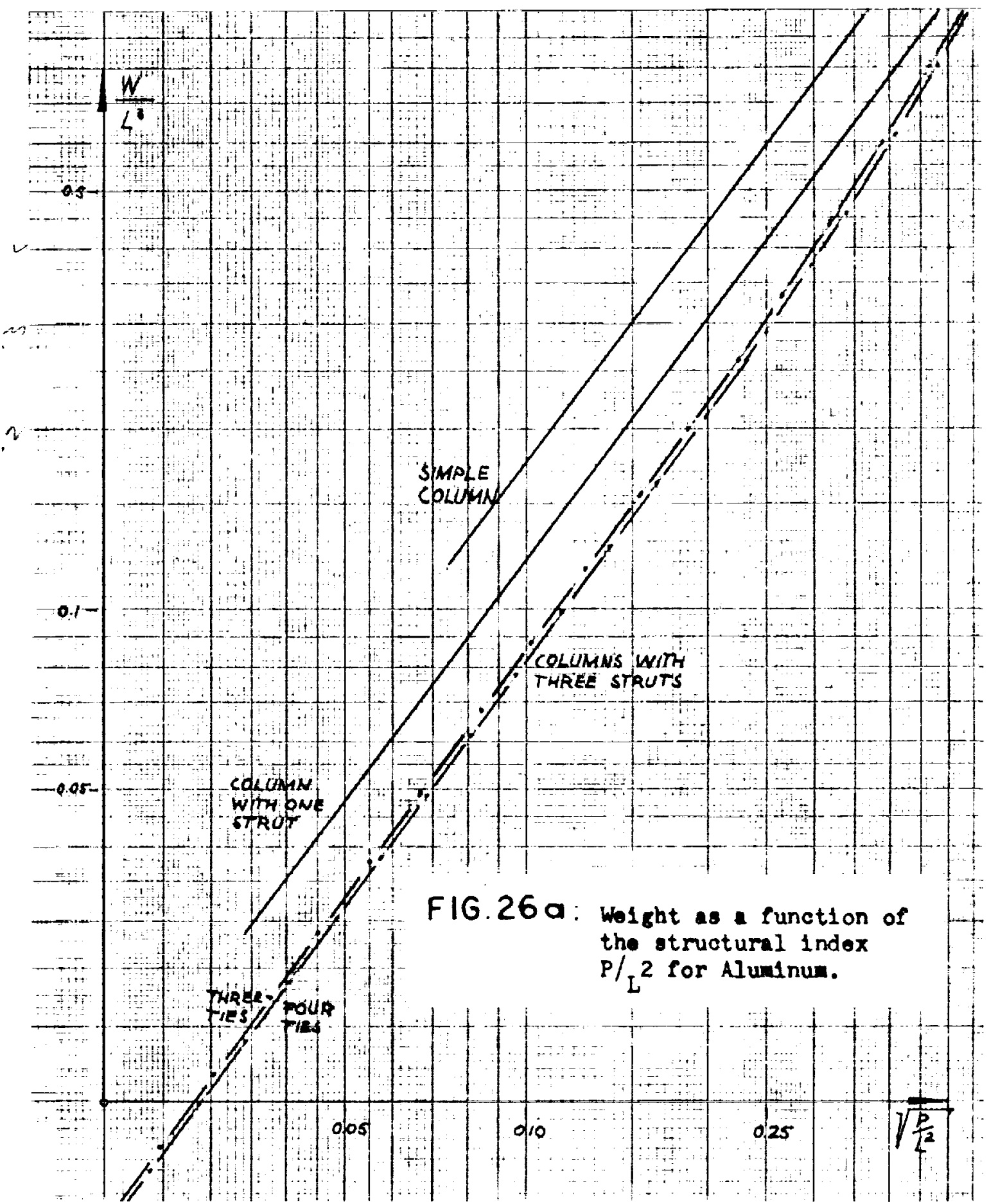




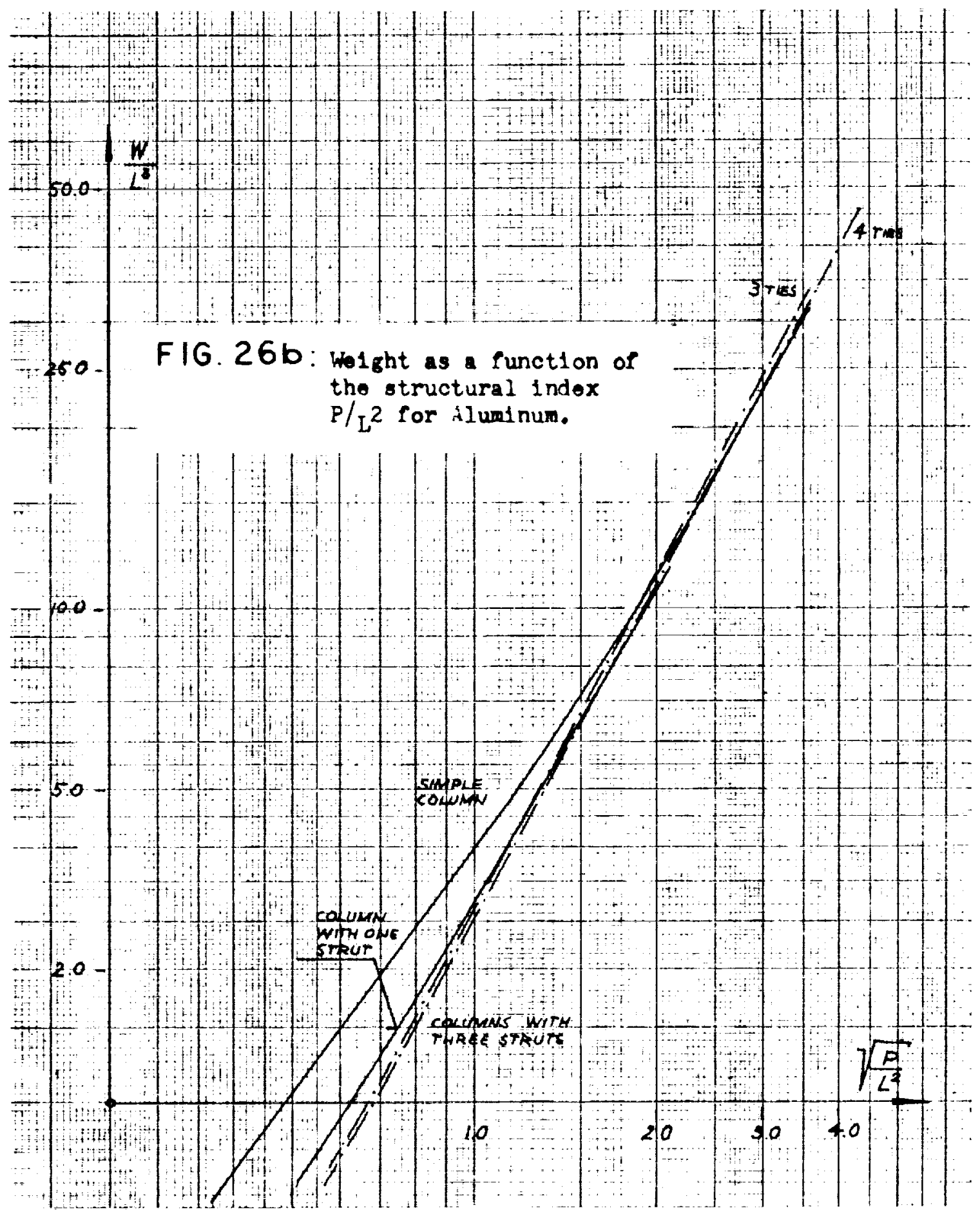




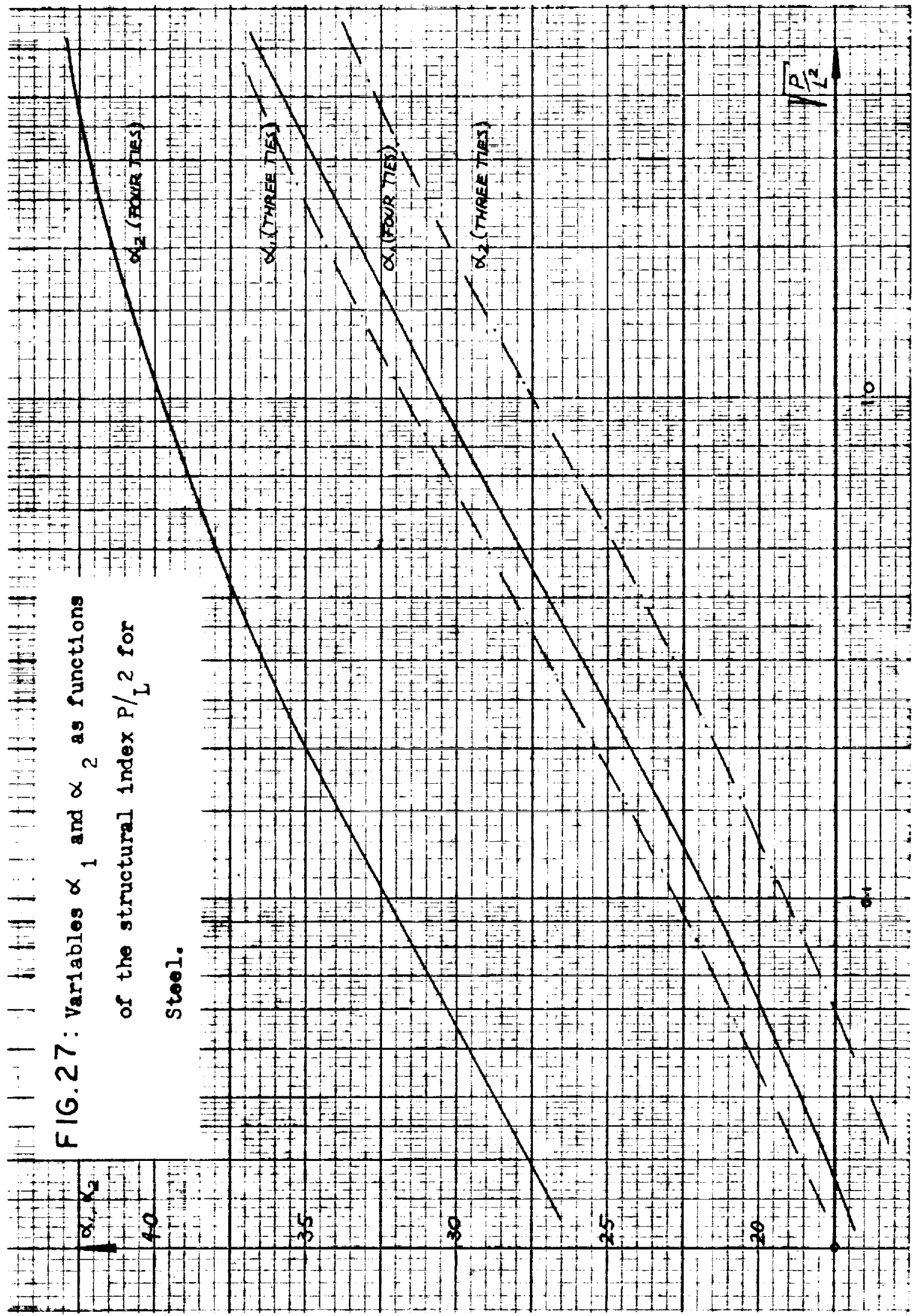




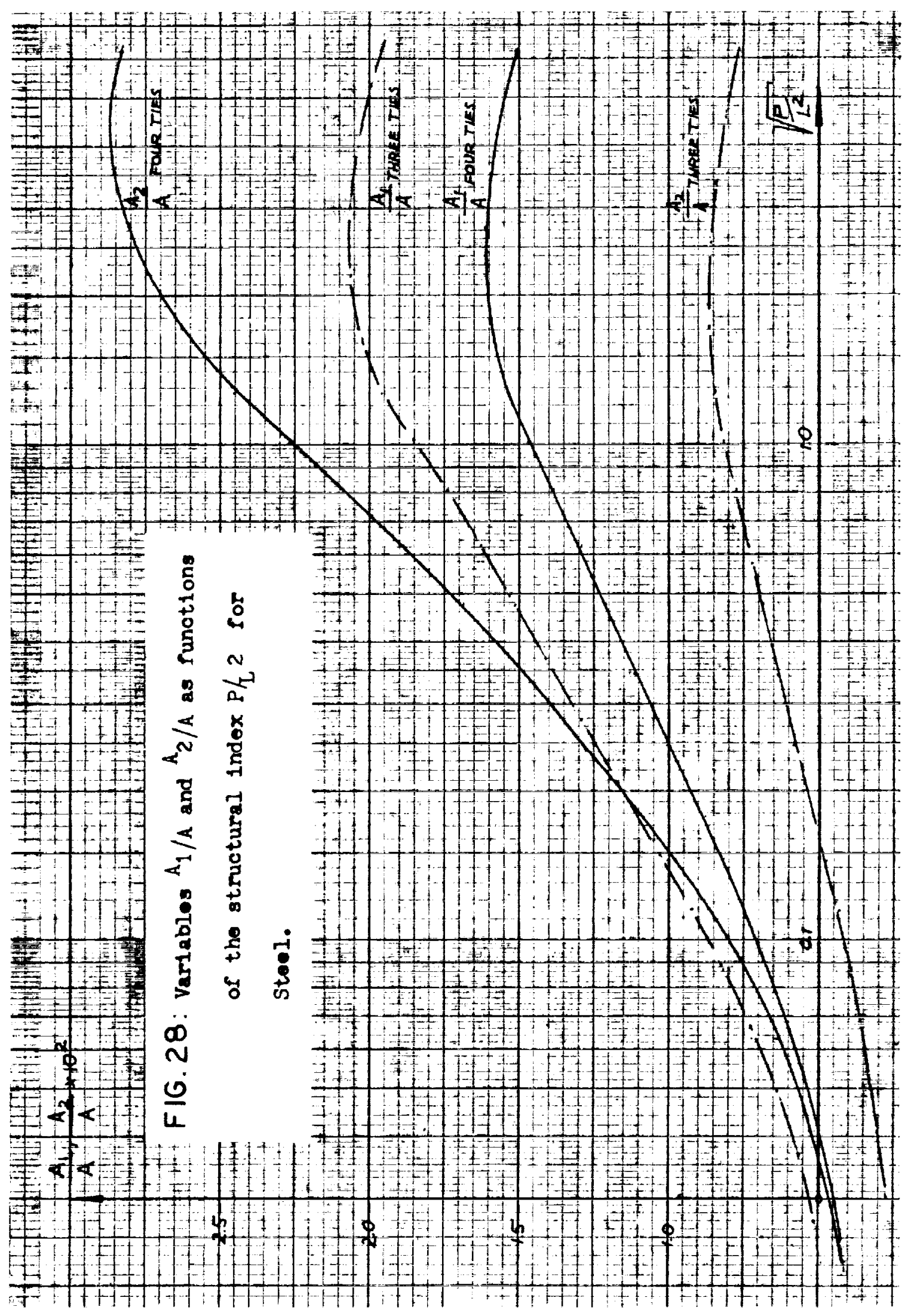




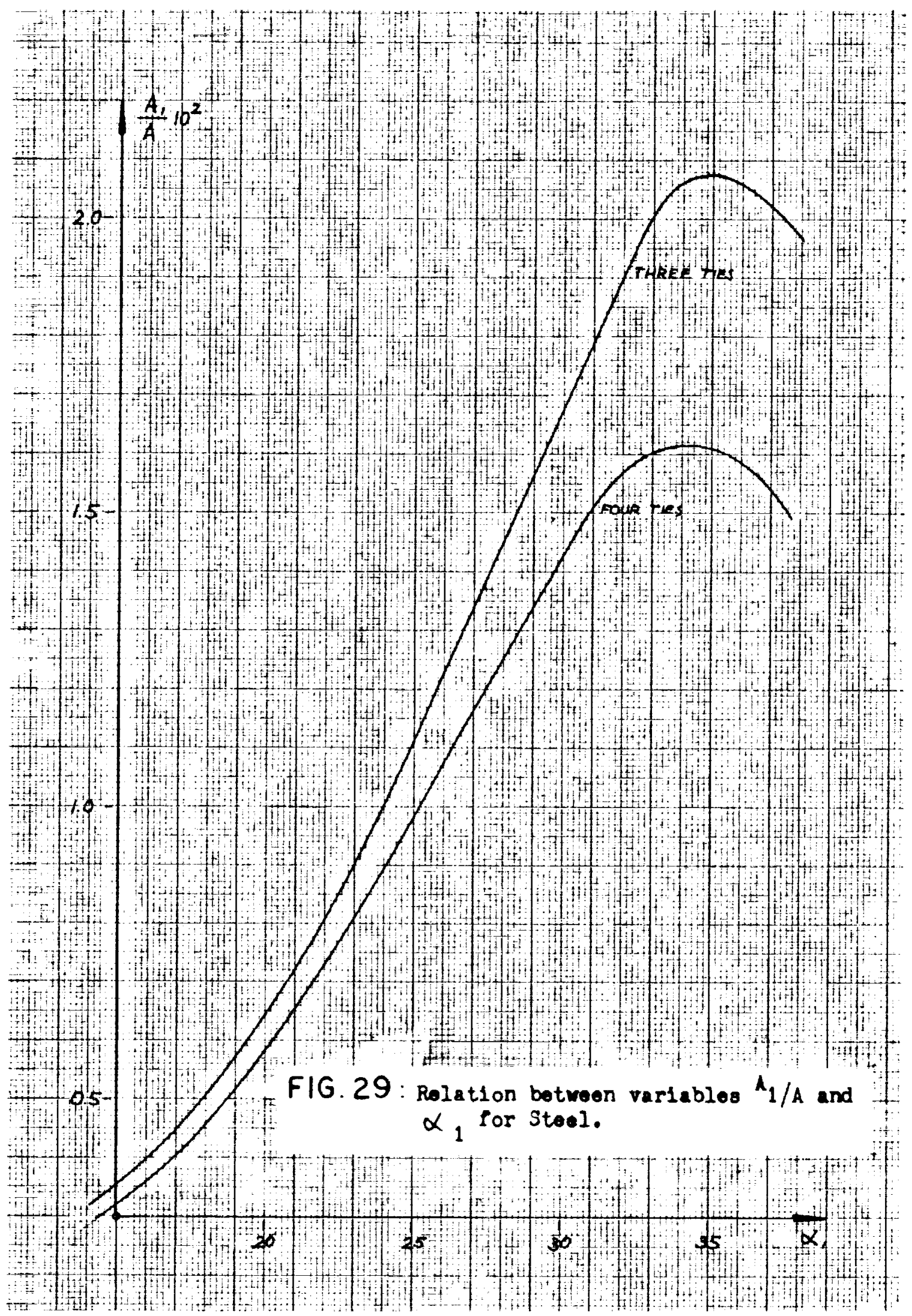




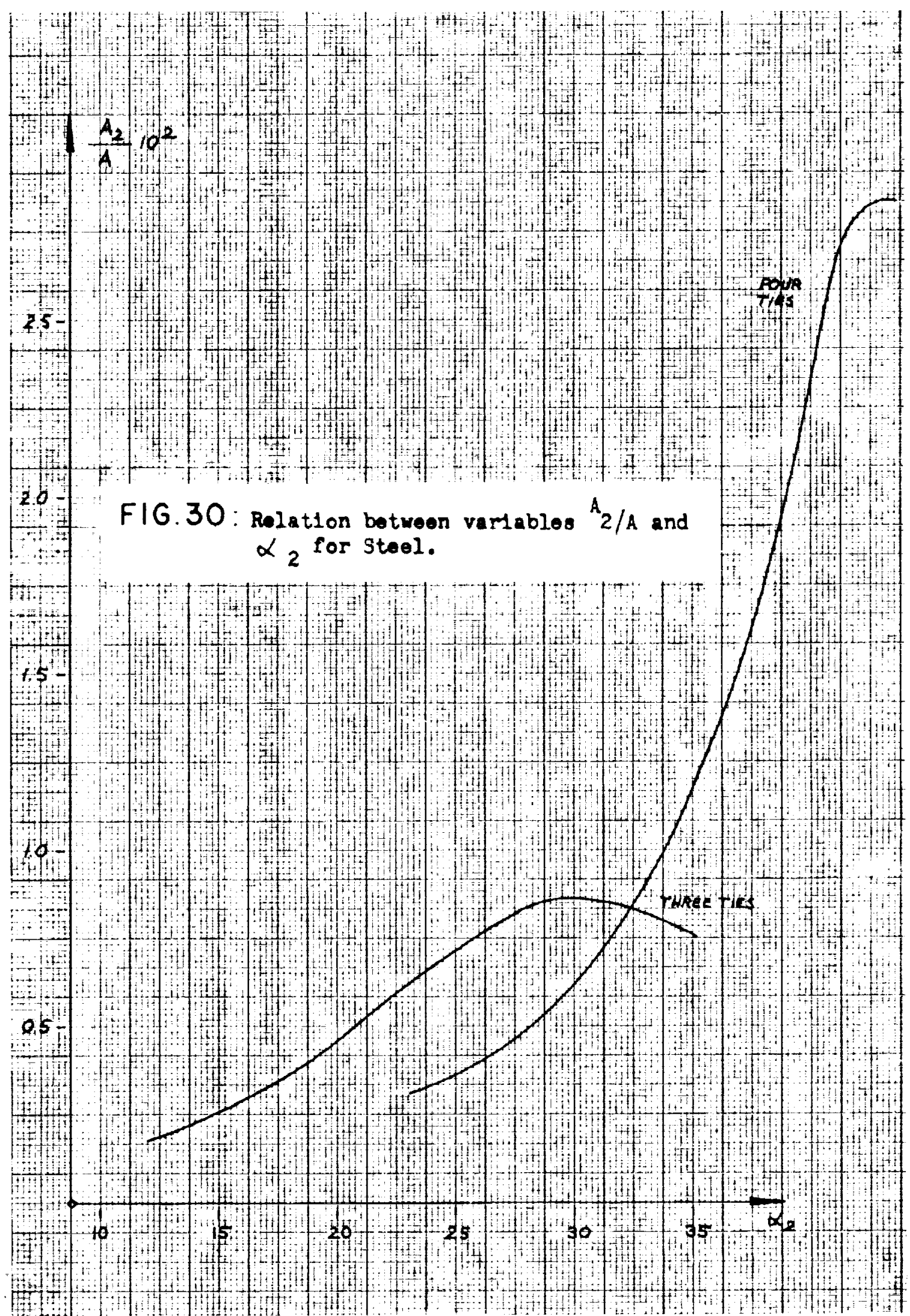




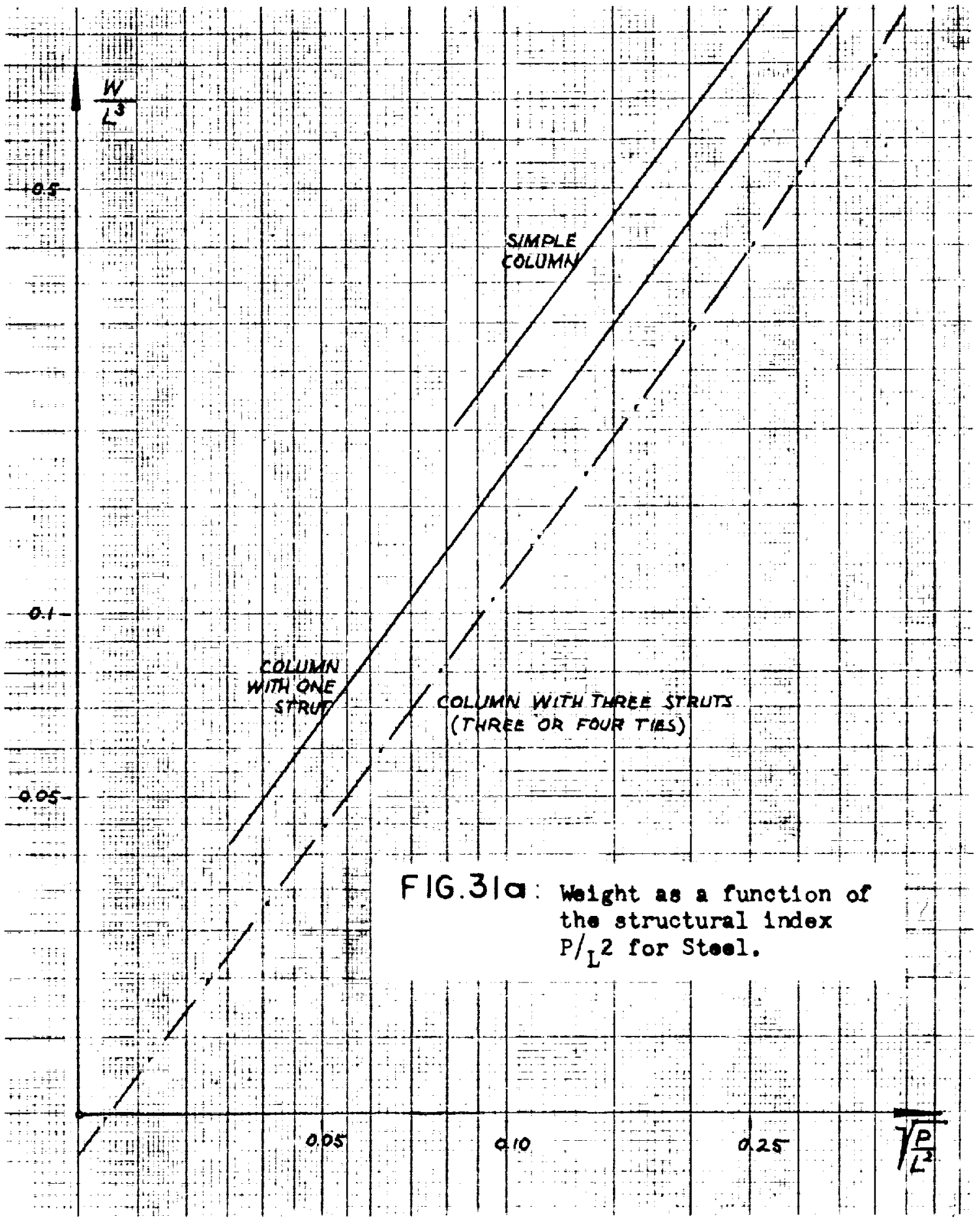




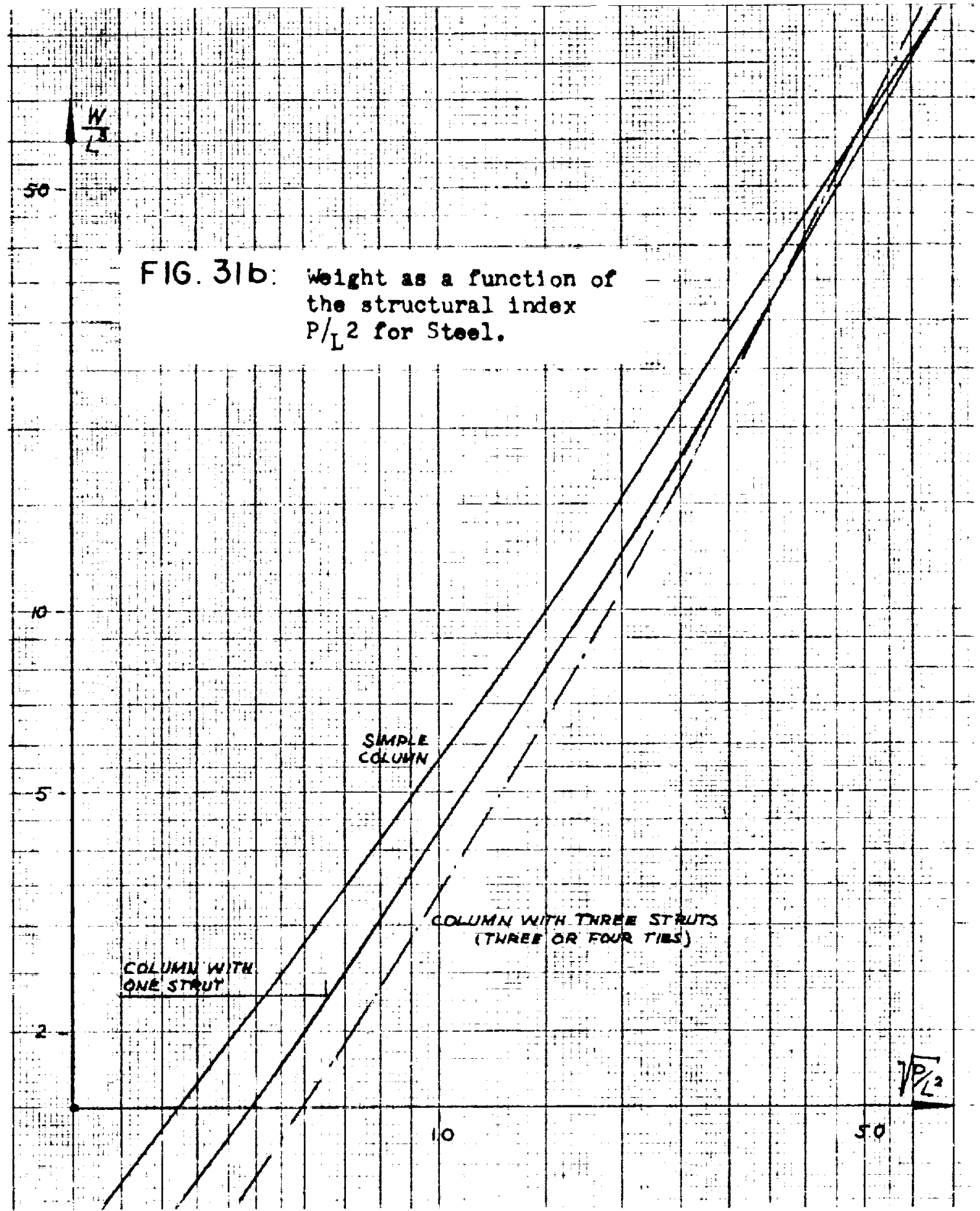




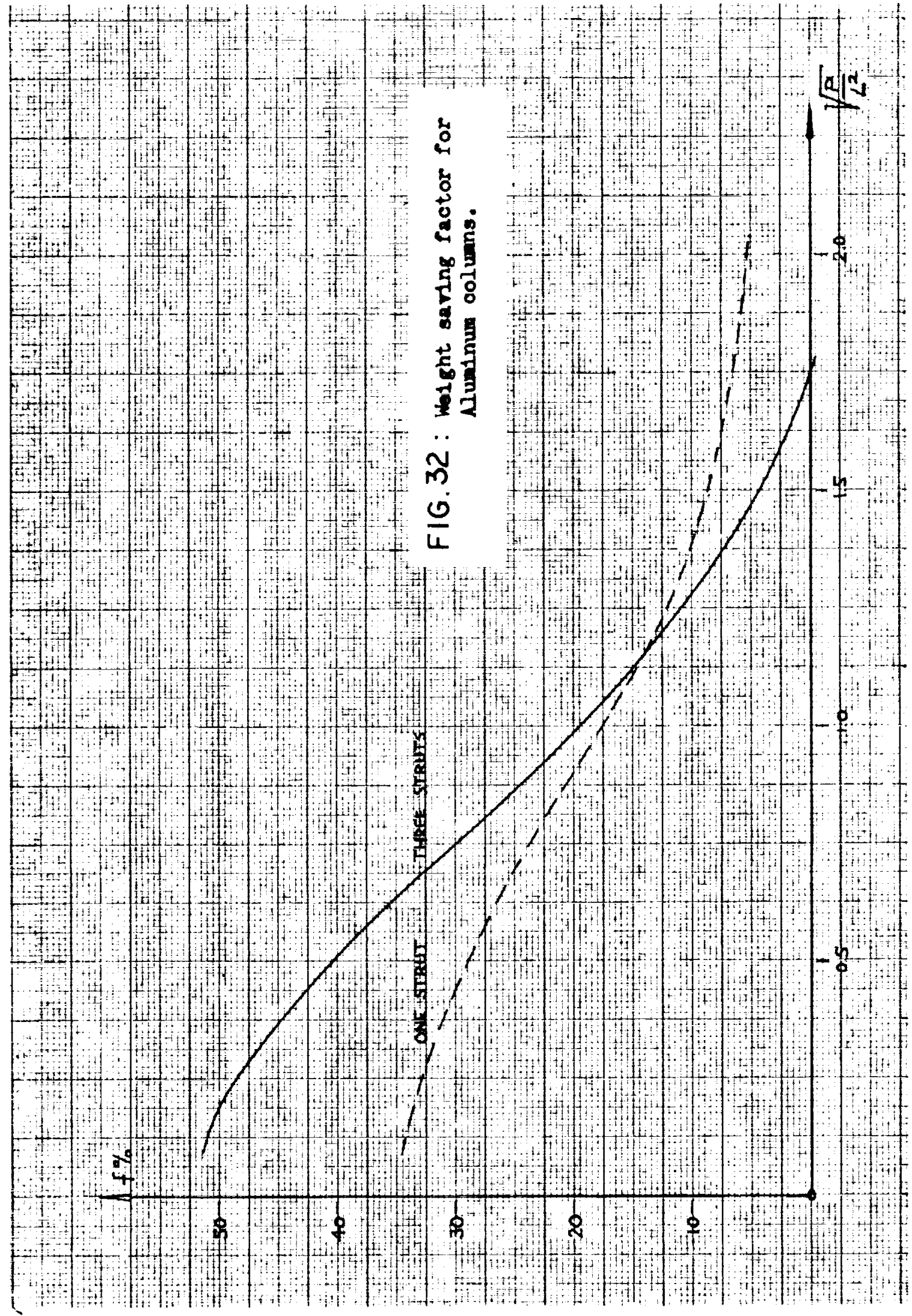




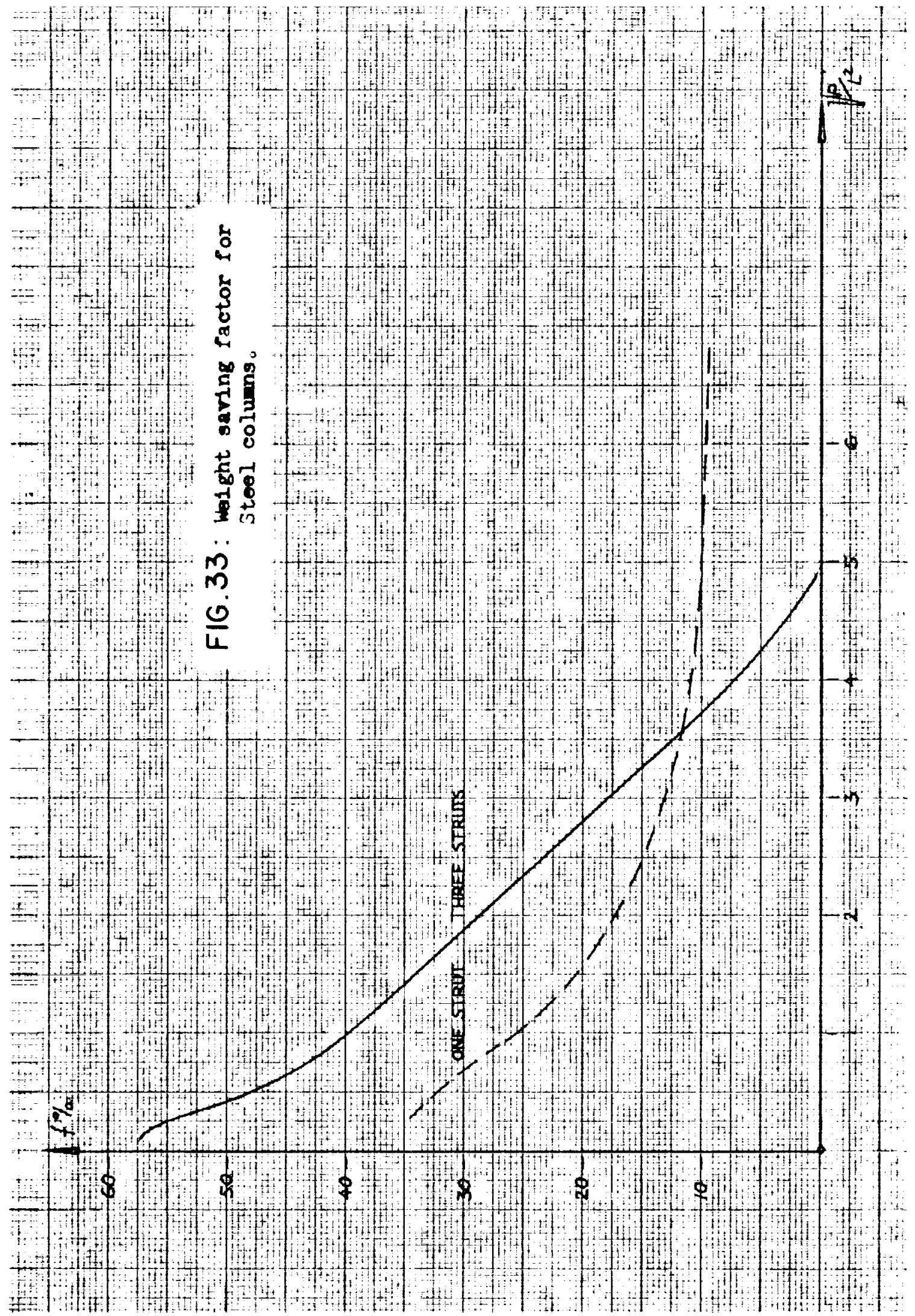


APPENDIX II

Computer Programs and Results 


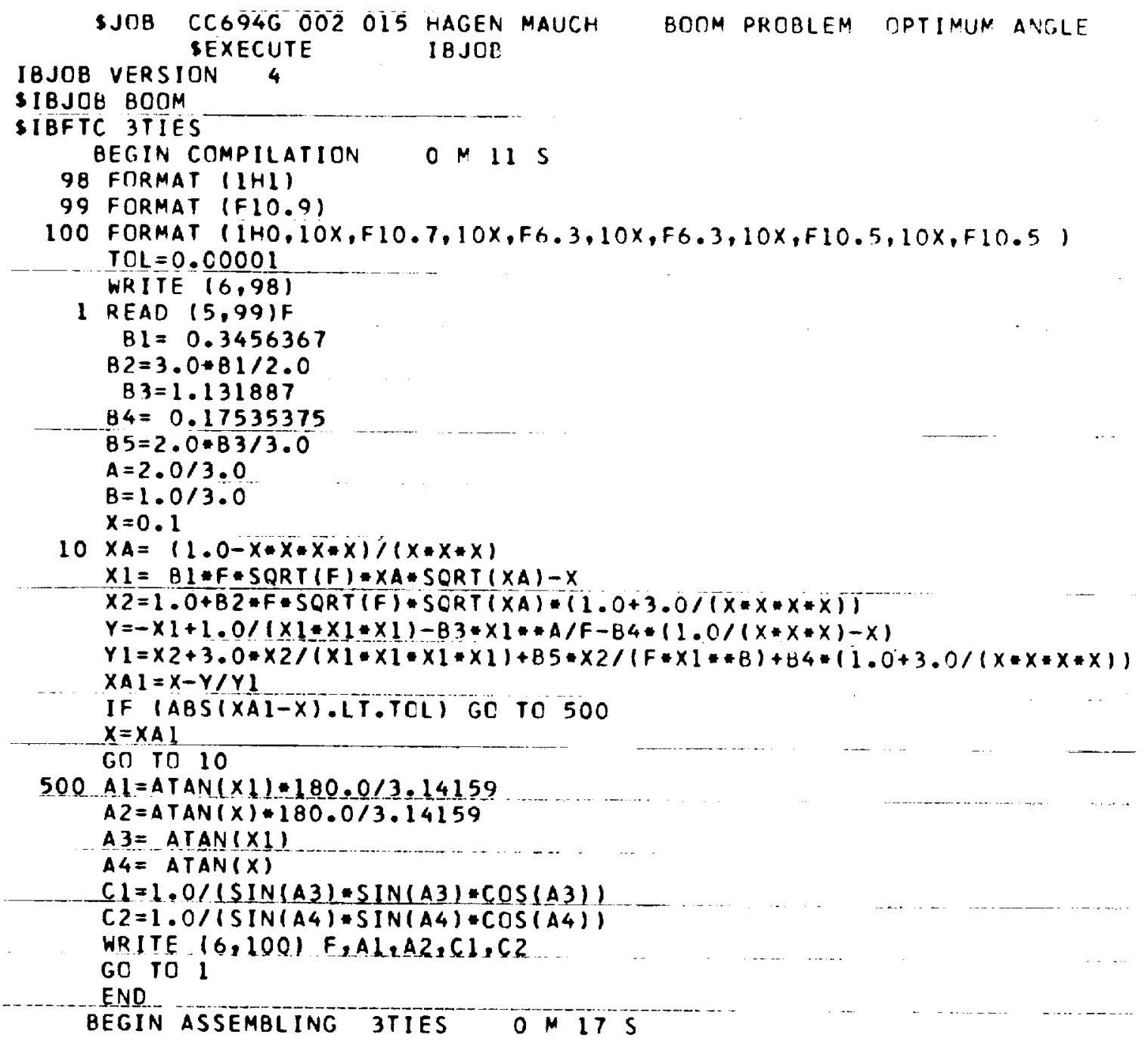




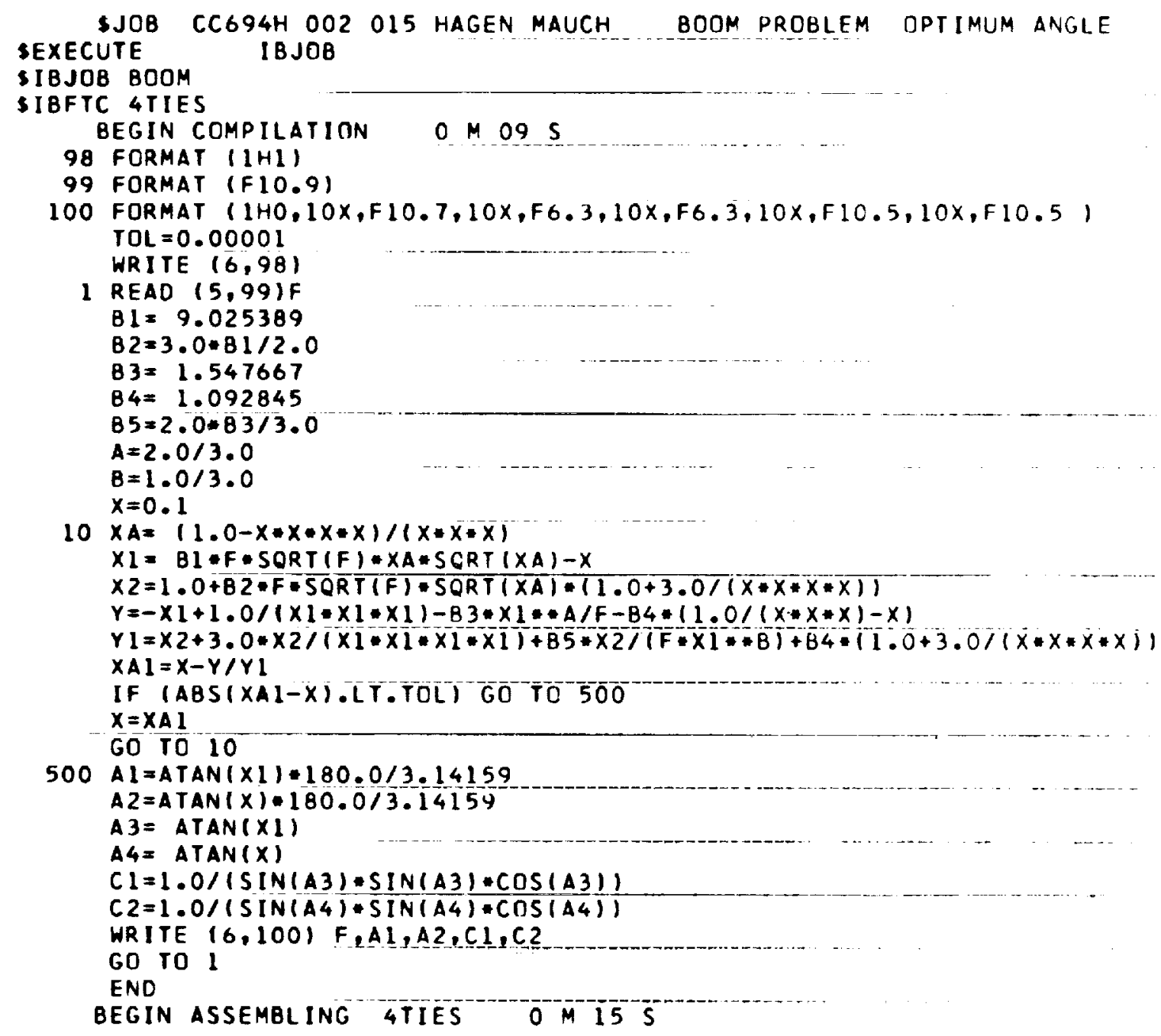



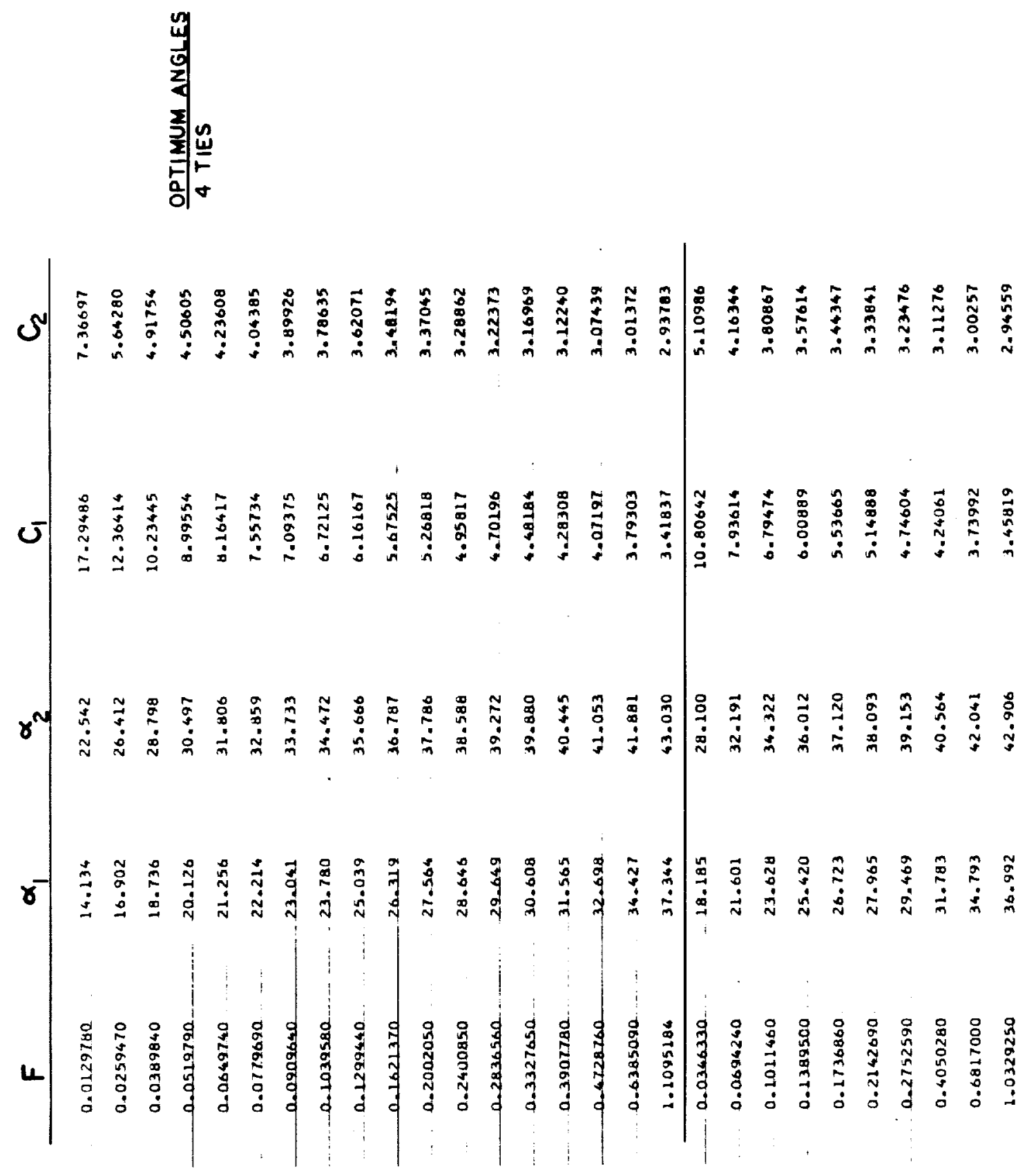

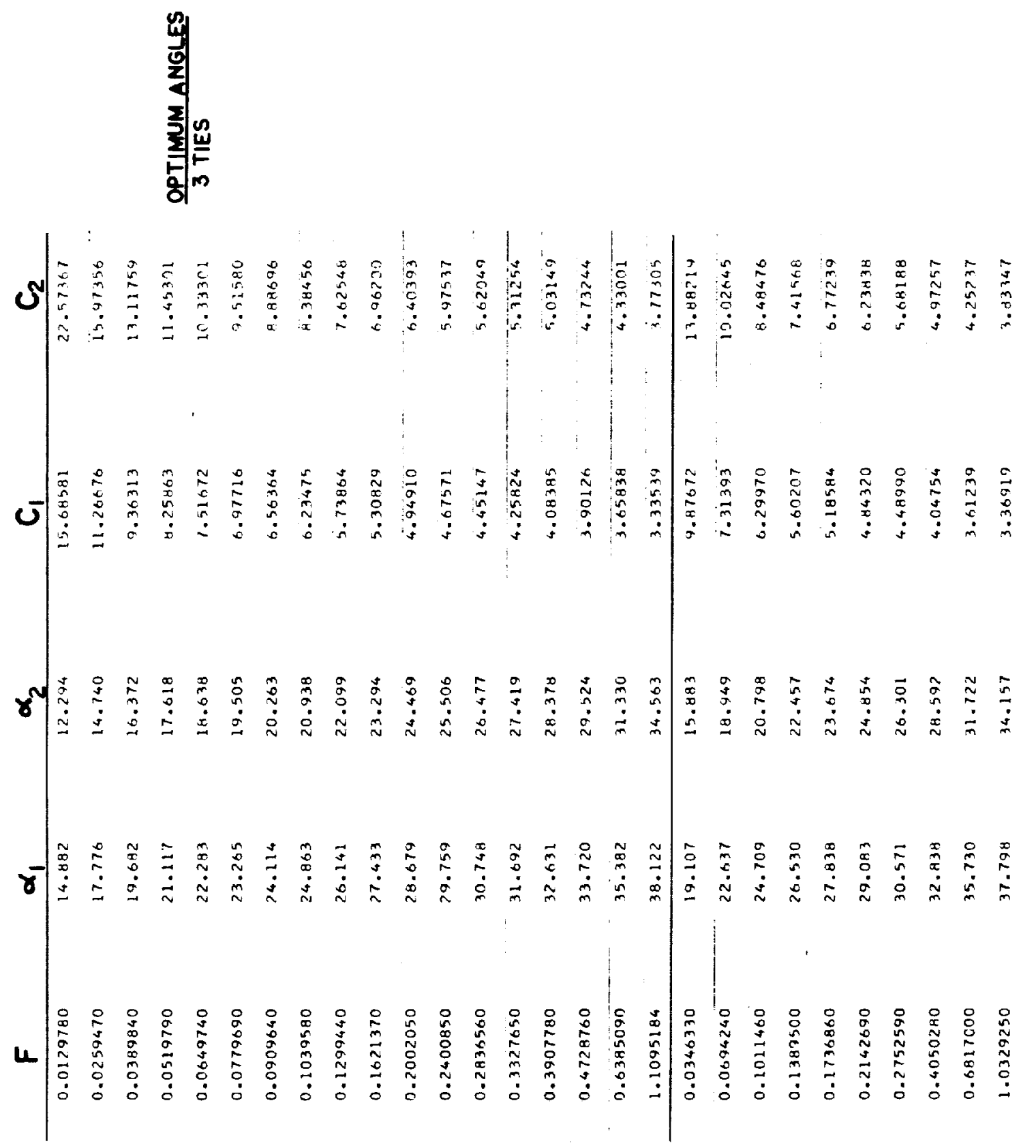


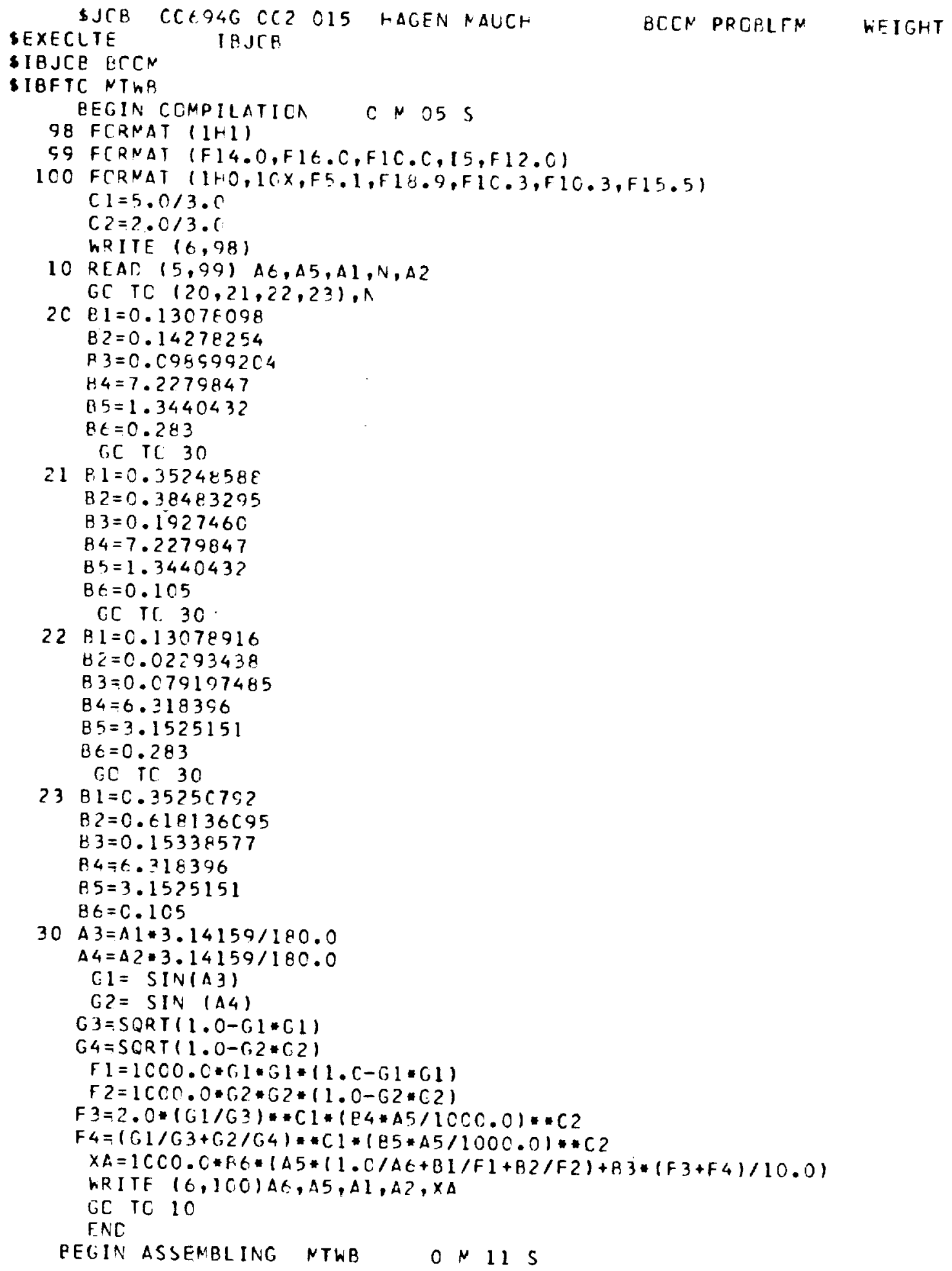




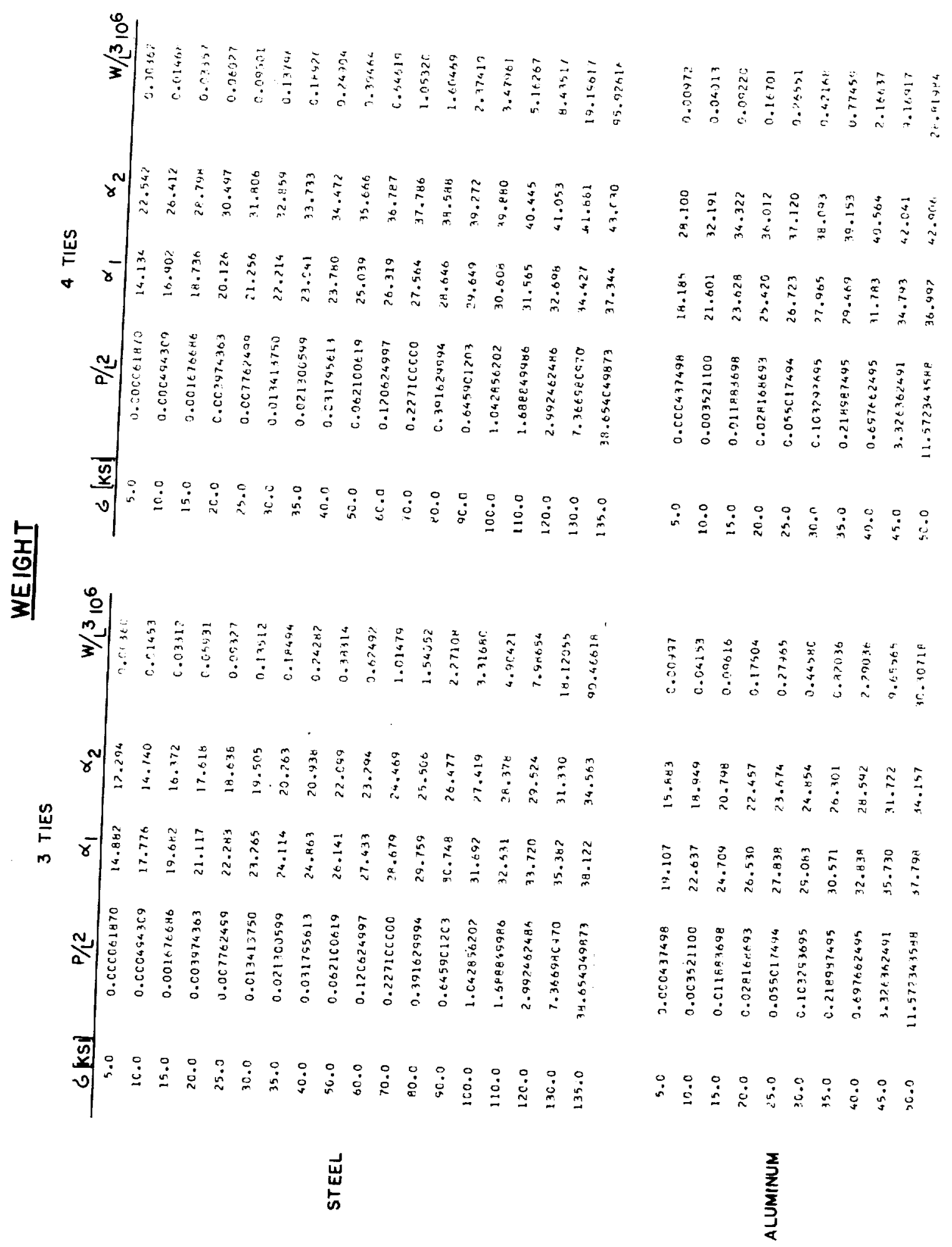


v

v

$\sim$ 